KU Leuven

Biomedical Sciences Group

Faculty of Medicine

KU LEUVEN

Department of Cellular and Molecular Medicine

\title{
FUNCTIONS OF SENSORY TRP CHANNELS IN VASCULAR RESPONSES TO CHEMICAL AND THERMAL STIMULI
}

Lucía ALONSO CARBAJO

Jury:

Promoter: Prof. Karel Talavera Pérez

Co-promoter: Prof. María Teresa Pérez García Chair: Prof. Rudi Vennekens

Jury members: Prof. Peter Vangheluwe

Prof. Félix Viana de la Iglesia
Dissertation presented in partial fulfilment of the requirements for the degree of Doctor in Biomedical Sciences 

Universidad deValladolid

PROGRAMA DE DOCTORADO EN INVESTIGACIÓN

BIOMÉDICA

TESIS DOCTORAL:

FUNCTIONS OF SENSORY TRP CHANNELS IN VASCULAR RESPONSES TO CHEMICAL AND THERMAL STIMULI

\author{
Presentada por Lucía Alonso Carbajo \\ para optar al grado de \\ Doctor/a por la Universidad de Valladolid
}

Dirigida por:

Prof. Karel Talavera Pérez

Prof. María Teresa Pérez García 

A mis padres $y$ a mi marido, Alejandro 



\section{Table of Contents}

List of abbreviations

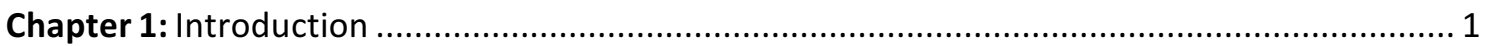

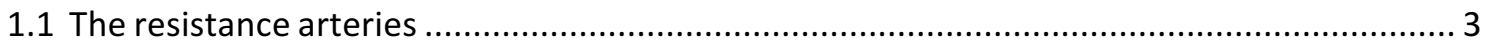

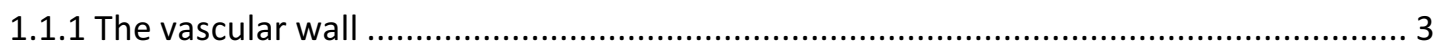

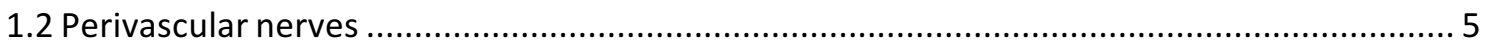

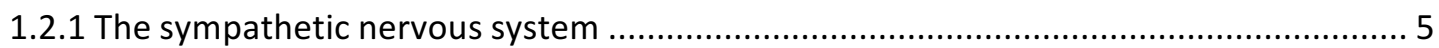

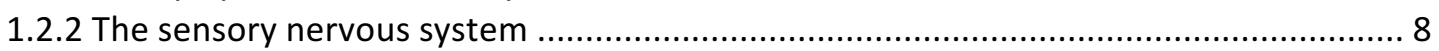

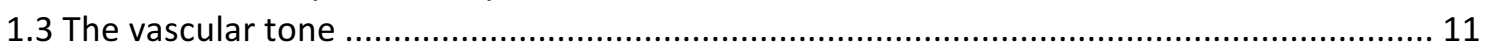

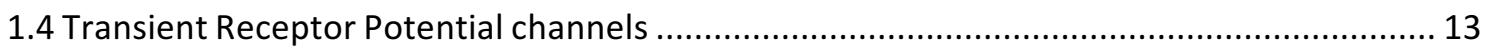

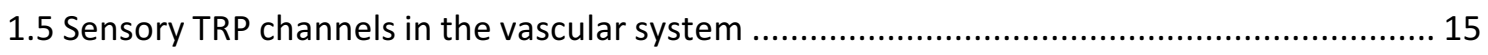

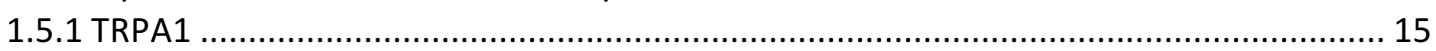

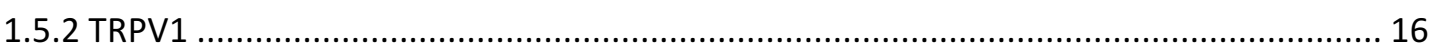

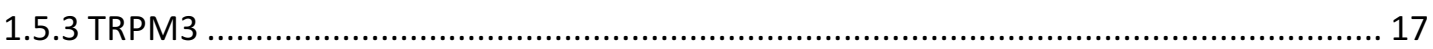

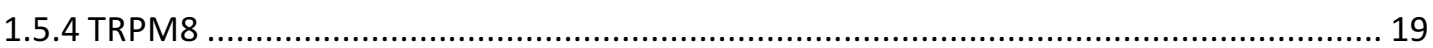

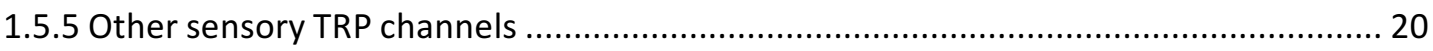

1.6 Modulation of vascular function by thermal stimuli: role of sensory TRP channels ............. 21

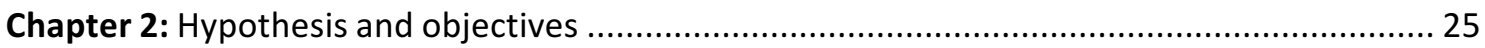

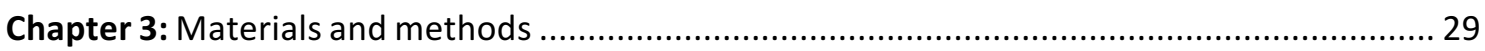

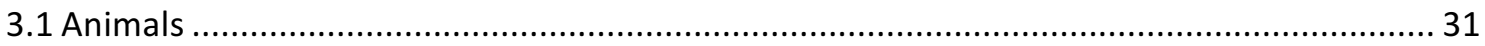

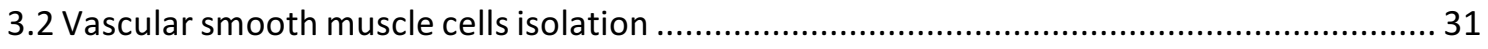

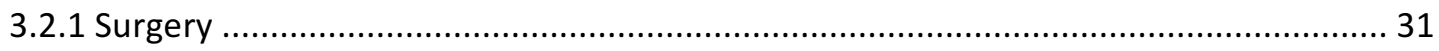

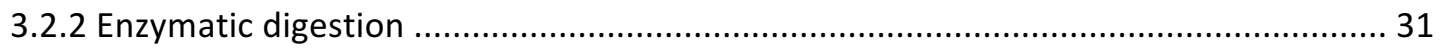

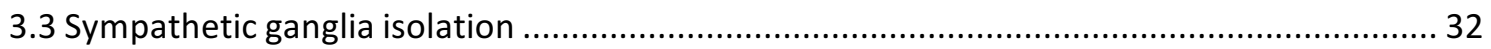

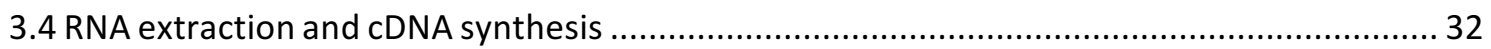

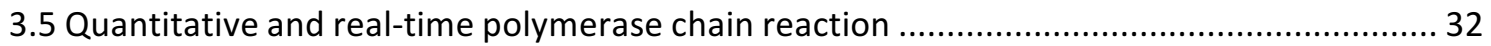

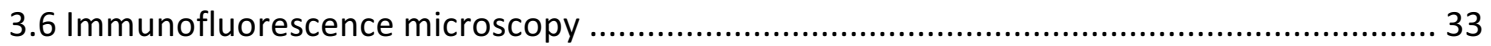

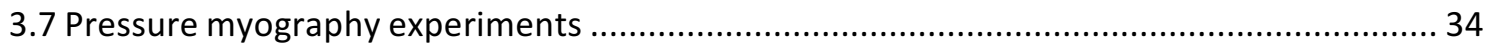

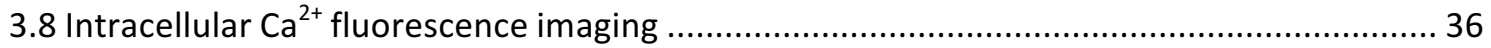

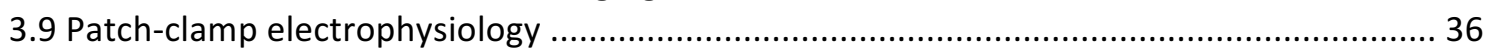

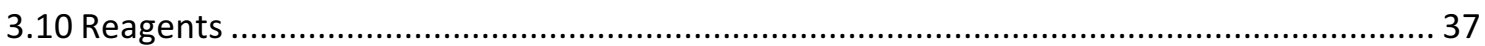

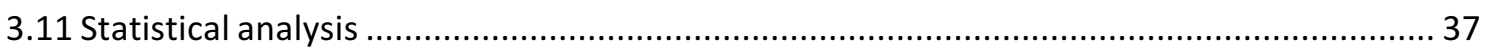

Chapter 4: Activation of the cation channel TRPM3 in perivascular nerves induces vasodilation

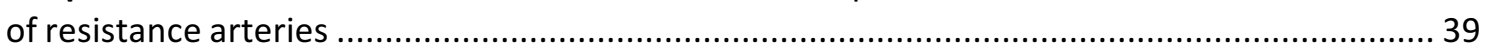

4.1 Expression pattern of TRPM family in mouse mesenteric arteries ..................................... 41

4.2 Localization of TRPM3 in perivascular nerves of mesenteric arteries .................................. 42

4.3 TRPM3 activation induces vasodilation mainly via stimulation of CGRP receptors .............. 48

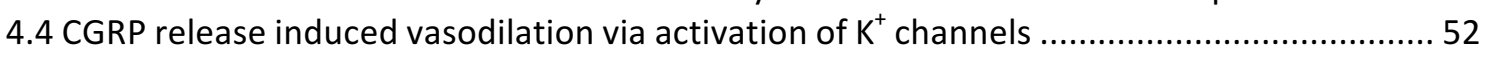

4.5 Sympathetic nerves are not implicated in TRPM3-mediated vasodilation ............................. 55

Chapter 5: Role of TRPA1 and TRPM8channels in vascular responses to cold ..........................5 59

5.1TRPA1 and TRPM8 channels are involved in intrinsic vascular responses to cold ..................61

5.2 Implication of the perivascular innervation in intrinsic vascular responses to cold..............66

Chapter 6: Discussion, general conclusions and future perspectives ..................................... 71

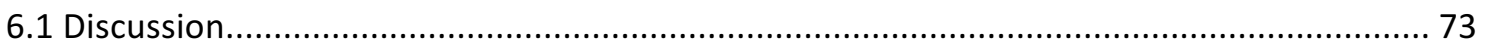

6.1.1 Activation of the cation channel TRPM3 in perivascular nerves induces vasodilation

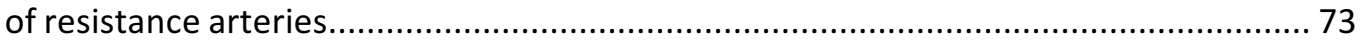

6.1.2 Role of TRPA1 and TRPM8channels in vascular responses to cold .............................. 77

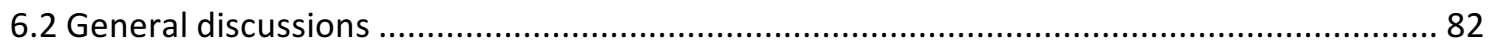

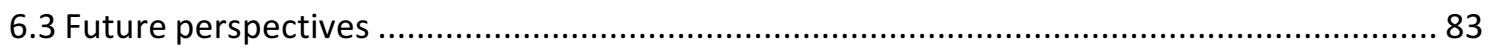

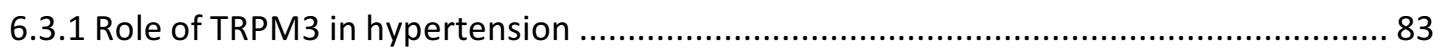

6.3.2 Cold shock response in 'in vivo' models: role of TRPA1 and TRPM8 channels .............. 85 


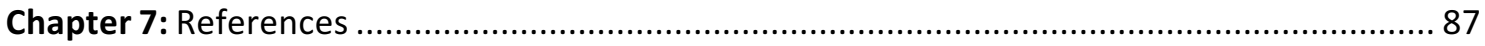

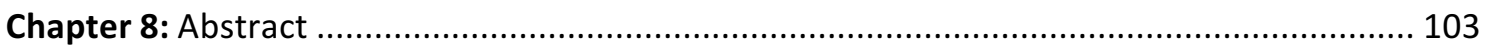

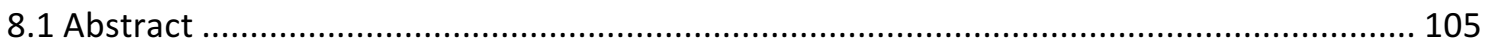

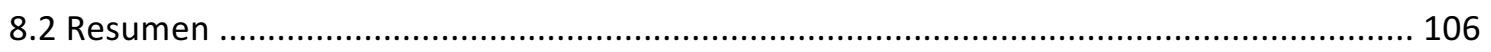

Acknowledgements, personal contribution and conflict of interest statements ................... 107

Chapter 9: List of publications and curriculum vitae .................................................... 109

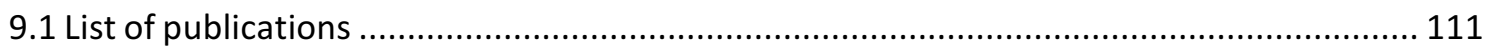

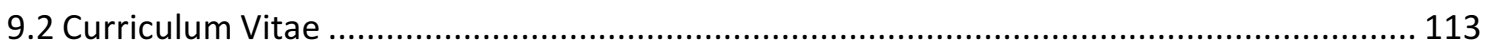




\section{List of abbreviations}

AC

$\mathrm{ACh}$

AITC

$\alpha$-SMA

Angll

ATP

BAPTA

ACTB

$\beta$-gal

$\mathrm{BK}_{\mathrm{Ca}}$

BPH

BPN

BSA

CAMP

CAT

CGRP

CHO

CLR

CNS

CO

Ct

DAG

DHEA

DIDS

DMSO

DRG

DTE

EC

$\mathrm{EC}_{50}$

EGTA

GAPDH

GPCR

HEK293T

HEPES

HPLC

iNOS

$\mathrm{K}_{\text {ATP }}$

$K_{\text {IR }}$

KO

Kv

L-DOPA

MLCK

MAPK

NA

NGF

Nif

NF-200

NMDG

NO
Adenylate cyclase

Acetylcholine

Allyl isothiocyanate

$\alpha$-smooth muscle actin

Angiotensin II

Adenosine triphosphate

1,2-bis(o-aminophenoxy) ethane- N,N,N',N'-tetraacetic acid

$\beta$-actin

$\beta$-galactosidase

$\mathrm{Ca}^{2+}$-dependent $\mathrm{K}^{+}$channels

Blood pressure high

Blood pressure normal

Bovine serum albumin

Cyclic adenosine monophosphate

Choline acetyltransferase

Calcitonin gene-related peptide

Chinese hamster ovary

Calcitonin-like receptor

Central nervous system

Cardiac output

Threshold cycle

Diacylglycerol

Dehydorepiandrosterone

4,4'-Diisothiocyano-2,2'-stilbenedisulfonic acid

Dimethyl sulfoxide

Dorsal root ganglion

1,4-Dithioerythritol

Endothelial cells

Half maximal concentration

Ethylene glycol-bis(2-aminoethylether)- $N, N, N^{\prime}, N^{\prime}$-tetraacetic acid

Glyceraldehyde 3-phosphate dehydrogenase

G protein-coupled receptors

Human embryonic kidney cells 293 SV40 T-antigen

4-(2-hydroxyethyl)-1-piperazineethanesulfonic acid

High-performance liquid chromatography

Inducible nitric oxide synthase

ATP-dependent $\mathrm{K}^{+}$channels

Inward rectifier $\mathrm{K}^{+}$channels

Knockout

Voltage-dependent $\mathrm{K}^{+}$channels

Levodopa

Myosin light chain kinase

Mitogen-activated protein kinases

Noradrenaline

Nerve growth factor

Nifedipine

Neurofilament 200

$\mathrm{N}$-methyl-D-glucamine

Nitric oxide 


\begin{tabular}{|c|c|}
\hline NTC & Non-template controls \\
\hline NTs & Neurotransmitters \\
\hline NT3 & Neurotrophin-3 \\
\hline PBS & Phosphate-buffered saline \\
\hline PFA & Para-formaldehyde \\
\hline PGP9.5 & Protein gene product 9.5 \\
\hline Phe & Phenylephrine \\
\hline $\mathbf{P I P}_{2}$ & Phosphatidylinositol 4,5-bisphosphate \\
\hline PKA & Protein kinase $\mathrm{A}$ \\
\hline PLC & Phospholipase C \\
\hline PNS & Peripheral nervous system \\
\hline PS & Pregnenolone sulfate \\
\hline PSS & Physiological saline solution \\
\hline qPCR & Quantitative real time polymerase chain reaction \\
\hline RAMP & Receptor activity modifying protein \\
\hline ROCK & Rho kinase \\
\hline ROS & Reactive oxygen species \\
\hline SCG & Superior cervical ganglion \\
\hline SEM & Standard error of the mean \\
\hline SMDS & Smooth muscle dissociation solution \\
\hline SNS & Sympathetic nervous system \\
\hline SPNS & Sensory peripheral nervous system \\
\hline SP & Substance P \\
\hline TH & Tyrosine hydroxylase \\
\hline TNF $\alpha$ & Tumor necrosis factor $\alpha$ \\
\hline TPR & Total peripheral resistance \\
\hline TRP & Transient receptor potential \\
\hline TRPA & Transient receptor potential ankyrin \\
\hline TRPC & Transient receptor potential canonical \\
\hline TRPM & Transient receptor potential melastatin \\
\hline TRPML & Transient receptor potential mucolipin \\
\hline TRPP & Transient receptor potential polycystin \\
\hline TRPV & Transient receptor potential vanilloid \\
\hline UTP & Uridine-5'-triphosphate \\
\hline VDCC & Voltage-dependent $\mathrm{Ca}^{2+}$ channels \\
\hline VSM & Vascular smooth muscle \\
\hline VSMC & Vascular smooth muscle cells \\
\hline
\end{tabular}




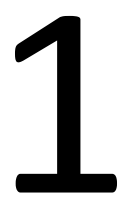

\section{INTRODUCTION}

Part of this literature was integrally published in Cell Calcium (volume 66: 48-61, 2017) of which Lucía Alonso-Carbajo is first author. 
Fine-tuned regulation of the arterial pressure is an essential physiological process determined by two important parameters, cardiac output (CO) and total peripheral resistance (TPR). The deregulation of the blood pressure can lead to cause severe vascular disorders over time and even sudden death. Multiple pathways including the autonomic nervous system, renin-angiotensin system, aldosterone, vasoactive substances and vascular responses to external changes affect CO and TPR to tightly regulate vascular functions and thus ensure appropriate blood flow along the body. Arteries with small diameters, also called resistance arteries, contribute significantly to the regulation and creation of resistance to blood flow in order to maintain a normal arterial pressure [1].

\subsection{The resistance arteries}

\subsubsection{The vascular wall}

Resistant arteries are normally in a partial contraction state which is known as basal tone, from which they can constrict further or dilate depending on the tissue demand for blood [2], allowing blood flow changes in response to local stimuli. When the resistance artery is dilated, the resistance is decreased and the local blood flow increases. On the contrary, the vasoconstriction of these small arteries increases the local resistance and decreases the blood flow to the tissues [1]. The wall of these arteries is formed by three layers: tunica intima, tunica media and tunica adventitia (Figure 1.1).

\subsubsection{Tunica intima}

The tunica intima is the innermost layer and it is composed of endothelial cells (EC) surrounded by a fine network of connective tissue. EC lining the vascular lumen of the vessel are sealed to each other by "tight junctions", restricting the diffusion of large molecules across the endothelium. The EC constitute a semipermeable layer that retains the escape of plasma and the blood cellular components in the circulation, and allow the circulation of nutrients between the blood and the tissues. The glycocalyx and the intercellular junctions play a crucial role in controlling the permeability of this layer. These structures are very permeable to gases and ions and also to other small molecules such as hormones and metabolites (glucose, amino acids, etc.), but prevent the diffusion 
of large molecules such as the plasma proteins. The endothelium also secretes vasoactive agents such as nitric oxide (NO) that can act on the adjacent vascular smooth muscle cells (VSMC) resulting in vasodilation $[1,3]$.

\subsubsection{Tunica media}

The tunica media is the middle layer of the vessel wall and is responsible for the mechanical activity of the artery. This layer contains VSMC that are helically embedded in an extracellular matrix mainly formed by elastin and collagen fibres; their activity contracts or dilates the artery. The tunica media is limited by the internal and external

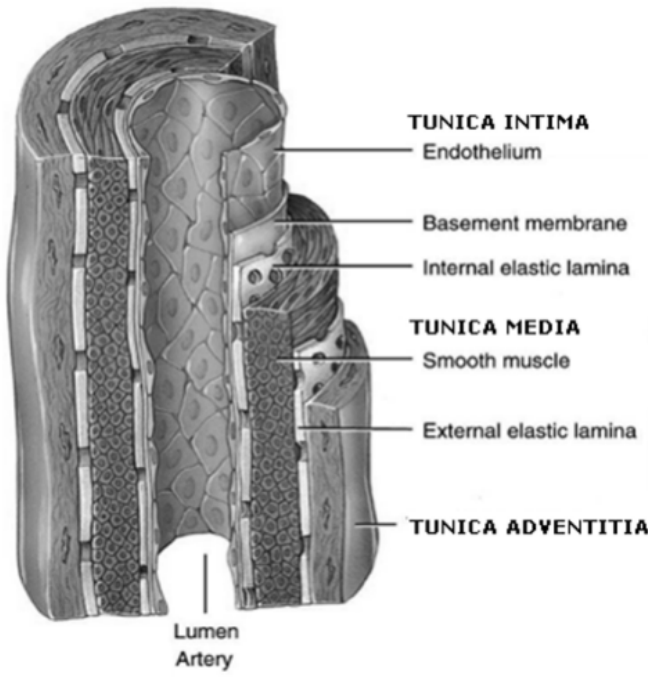

Figure 1.1. Schematic description of the structure of a resistance artery wall formed by three layers: tunica intima, tunica media and tunica adventitia (Wagenseil et al., 2009).

mainly composed of connective tissue sheath with no distinct outer border. An external elastic lamina separates the tunica media from the adventitia. The principal function of the adventitia is to keep the vessel attached to the surrounding tissue. It also contains collagen fibres that serve as support for fibroblasts and small vessels to provide nutrients to the wall of large vessels (the "vasa vasorum", literally "vessels of vessels") and very importantly, sympathetic and sensory fibre terminals. Release of neurotransmitters (NTs) by activation of these fibres contributes to the regulation of the local vessel resistance and the blood flow $[1,5]$. 


\subsection{Perivascular nerves}

Resistance arteries are primarily innervated by the sympathetic and sensory neurons which can release different NTs (Figure 1.2). These NTs bind to specialized receptors such as $\alpha$-adrenergic receptors and G protein-coupled receptors (GPCR) present in VSMC and EC. The fine balance between the influence of the sympathetic and sensory nervous systems tightly regulates the vascular tone [6].

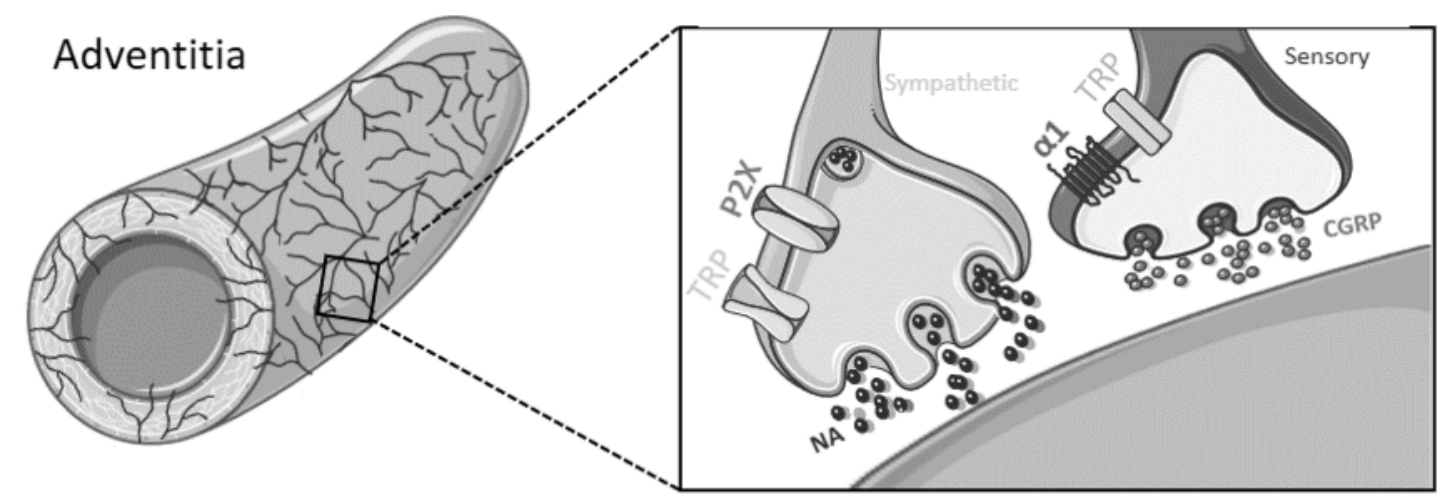

Figure 1.2. Anatomical location of perivascular sympathetic and sensory nerves. Perivascular nerves are located in the adventitia and can make direct contact with VSMC or EC. Purinergic and transient receptor potential (TRP) channels expressed in both types of nerves are involved in the release of multiple NTs. While efferent sympathetic fibres can release noradrenaline, sensory nerve axons are able to release calcitonin gen-related peptide (Adapted from Westcott, E.B. et al., 2013).

\subsubsection{The sympathetic nervous system}

The presence and functional role of parasympathetic perivascular nerves is poorly defined relative to those of sympathetic or sensory perivascular nerves. While in some vascular beds, parasympathetic nerves may play a minor role in vasomotor function, in most vessels there is no presence of these innervation [7]. Blood vessels are richly innervated by sympathetic vasoconstrictor nerves, constituting a key system to control the total peripheral resistance. The sympathetic nerves arise from little collections of small neuronal clusters located ventral and lateral to the spinal cord [8].

\subsubsection{Structure and action mechanism of sympathetic nervous system}

The sympathetic nervous system (SNS) is formed by clusters of neuron cell bodies called ganglia. Sympathetic ganglia are divided into two main groups, prevertebral and 
paravertebral, depending on their localization in the body. Prevertebral ganglia also called collateral ganglia innervate organs of the abdominal region and are lying between the paravertebral ganglia and the target organ, whereas paravertebral ganglia are located ventral and lateral to the spinal cord forming the sympathetic chain ganglia. The chain extends from the upper neck down to the coccyx.

In mammals, there are 22 pairs of these ganglia: 3 in the cervical region, 11 in the thoracic region, 4 in the lumbar region, and 4 or 5 in the sacral region. The superior ganglia innervate the head, and the middle innervate the neck, heart and upper limbs. The thoracic sympathetic ganglia are connected to the prevertebral ganglia that innervate the trunk region, and finally the lumbar and sacral sympathetic ganglia innervate the pelvic floor and lower limbs.

There are two types of neurons involved in the transmission of any signal through the sympathetic system: pre-ganglionic and post-ganglionic. The chemical transmission in the sympathetic system appears simple: preganglionic nerves originate from the spinal cord travel to a paravertebral ganglion or to a prevertebral ganglion in the case of the thoracic preganglionic neurons, where they synapse with a postganglionic fibre, which extends to an effector tissue. The distance between pre- and postsynaptic membranes can be quite large compared with typical synapses. For instance, the gap between cell membranes of a typical chemical synapse is $30-50$ nanometres, whereas in blood vessels the distance between the synaptic end of the post-ganglionic axons and the vascular tissue is often greater than 100 nanometres or, in some cases, 1-2 $\mu \mathrm{m}$. Also, unlike classical synapses, there is not a single site of neurotransmitter release from sympathetic nerves. Instead, neurotransmitter is released "en passant" from varicosities along the efferent axons and over a widespread area of the target tissue. In this way, a relatively small number of neurons can exert control of large tissue areas. The neurotransmitter released at the synapsis between pre- and post- ganglionic fibres is acetylcholine (ACh), alone or in combination with other peptide co-transmitters. These NTs activate nicotinic and peptidergic receptors on postganglionic neurons. The activation of these postganglionic neurons lead to the release of noradrenaline (NA), again alone or in combination with other NTs on the target organs (Figure 1.3). 
NA is formed by a complex process whereby tyrosine is catalyzed by the enzyme tyrosine-hydroxylase (TH) resulting in levodopa (L-DOPA). L-DOPA is transformed to dopamine due to the activity of DOPA decarboxylase and stored in synaptic vesicles. Within these vesicles, dopamine- $\alpha$-hydroxylase is the responsible for transforming dopamine into the final NA, which triggers contraction of the VSMC.
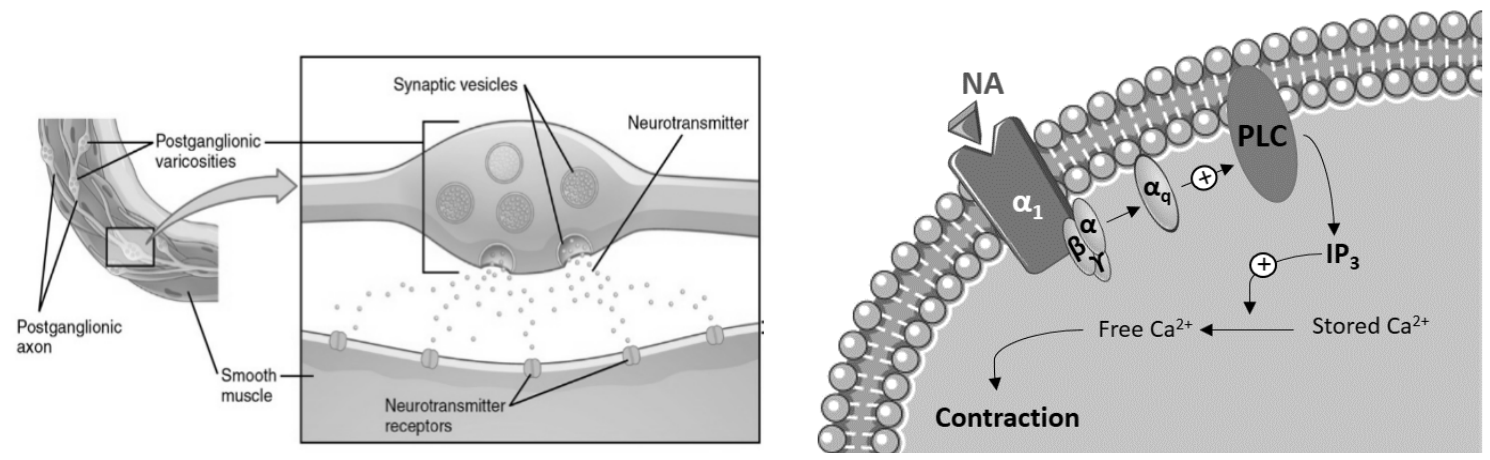

Figure 1.3. Sympathetic neuromuscular transmission. Noradrenaline secreted by postganglionic neurons binds to $\alpha_{1}$-receptors located in VSMC leading to vasoconstriction via phospholipase C pathway (Levick J.R., 2003).

However, neurotransmission is more complex, because multiple peptides are released, affecting different receptors on the target cell. In addition, all these NTs are selfregulatory, in the sense that they act on presynaptic receptors located on their own axon terminals [9].

Owing to the large gaps between autonomic nerve terminals and their effector cells, NTs tend also to act slowly or become inactivated. However, the widespread nature of these connections, together with the existence of low-resistance connections among VSMC, allow many cells responding as a group upon activation of only one cell.

\subsubsection{Role of sympathetic nervous system in vascular function}

The SNS is responsible for regulating many homeostatic mechanisms in the organisms. In resistance arteries, sympathetic vasoconstrictor fibres are tonically active releasing NA that binds mainly to $\alpha_{1}$-adrenergic receptors located in the VSMC, resulting in a constriction of the blood vessels [10]. This means that a reduced sympathetic vasomotor activity causes vasodilatation [7]. In addition, VSMC also express $\beta_{2}$-adrenoreceptors, whose activation by NA may induce arterial dilation [11]. However, the predominant 
action of NA released from sympathetic nerves of resistance vessels is consistently with a vasoconstriction, so that the physiological role of $\beta_{2}$-adrenoreceptors in the control of vascular tone remains to be established [6].

Alterations of the sympathetic regulation of vessels are involved in some diseases, such as Raynaud's syndrome. This phenomenon is associated with a hyperactivation of the SNS causing peripheral vasoconstriction, leading to tissue hypoxia. This hyperactivation, that normally occurs in response to low ambient temperature and is directed to avoid heat loss through the skin, is usually followed after a few minutes by a "paradoxical" vasodilation in healthy patients. Both the activation of the sympathetic neurotransmission together with the local release of vasodilator substances activated by tissue hypoxia are responsible for the read noses and ears when patients are exposed to very low temperatures for prolonged times. This vasodilation does not happen in Raynaud's patients. Possible alterations may include the increased sensitivity to cold of the adrenergic receptors, increased levels of locally released, systemically circulating vasoconstrictors or a deficiency or increased degradation of NO due to augmented oxidative stress. In any case, the only available treatment to date is the sympathetic denervation of cutaneous vessels [12].

A role of the SNS in the treatment of essential hypertension has also been proposed. Several studies have found significantly higher plasma NA levels in hypertensive subjects compared to healthy patients [13]. Furthermore, the association of obesity, hypertension, insulin resistance, and type-2 diabetes can be partially explained by interactions between insulin and the SNS [14]. This has led to a renewed interest in $\alpha$ adrenergic inhibitory approaches for the treatment of hypertension and other cardiovascular and metabolic diseases $[15,16]$.

\subsubsection{The sensory nervous system}

Sensory peripheral nerves are associated with VSMC. The expression of neurotrophic factors such as nerve growth factor (NGF) and neurotrophin-3 (NT3) in blood vessels is thought to be important to maintain the survival of growing axons before they arrive at their peripheral targets, which provide an independent source of neurotrophins [17]. In mutant embryos lacking sensory nerves, arteries fail to properly differentiate, while in 
those containing disorganized nerves the trajectory of blood vessel branching is altered to follow the nerves [18].

\subsubsection{Structure and action mechanism of sensory nervous system}

The sensory peripheral nervous system (SPNS) is formed mainly by nerves that collect information from receptors present in their terminals. Unlike sympathetic nerves, sensory nerves are capable of both antidromic conduction in response to a short reflex and orthodromic conduction that is caused by a long-term reflex, thereby enabling their participation in local axon reflexes independent of efferent signalling from the cell body.

In a short reflex, these sensory neurons are able to monitor conditions inside and outside of the body and synapse directly in a peripheral ganglion with other nerve fibre. On the contrary, in long-term reflexes the sensory neurons send this information to the central nervous system (CNS) that would be further processed in the peripheral ganglion (Figure 1.4). Thus, noxious stimuli can cause antidromic stimulation of sensory nerves, leading to neurotransmitter release and vasodilation, in addition to the sensation of pain (the long-term reflex, orthodromic).

Sensory nerves endings are free dendrites that extend into the target tissue. These free nerves endings are able to sense stimuli including pain, heat and cold. After the blockade of cholinergic and noradrenergic transmissions, blood vessels can still actively be dilated in response to nerve stimulation. There is strong evidence that these non-adrenergic and non-cholinergic vasodilator neurons are sensory fibres that secrete peptides such as the calcitonin gene-related peptide (CGRP) and substance P (SP). In the vascular system, CGRP has been identified as the main neurotransmitter released from sensory neurons [19]. These NTs bind to their specific receptors present in the media and intima layers of the blood vessels modulating the arterial function.

CGRP is a 37-amino acid neuropeptide that has two major forms ( $\alpha$ and $\beta$ ). $\alpha$ CGRP is primarily localized in the perivascular innervation and considered to be the major vascular form, as compared with the $\beta$ form. This peptide usually binds to a calcitoninlike receptor (CLR) that is linked to the Receptor Activity Modifying Protein (RAMP) which is necessary for increasing cyclic adenosine monophosphate (CAMP) production via the adenylate cyclase (AC) [20] and may therefore lead to protein kinase A (PKA)mediated activation of $\mathrm{K}^{+}$channels in VSMC and consequent hyperpolarization. aCGRP 
can also control the expression of the mitogen-activated protein kinases (MAPK) pathway and inducible nitric oxide synthase (iNOS) [21].

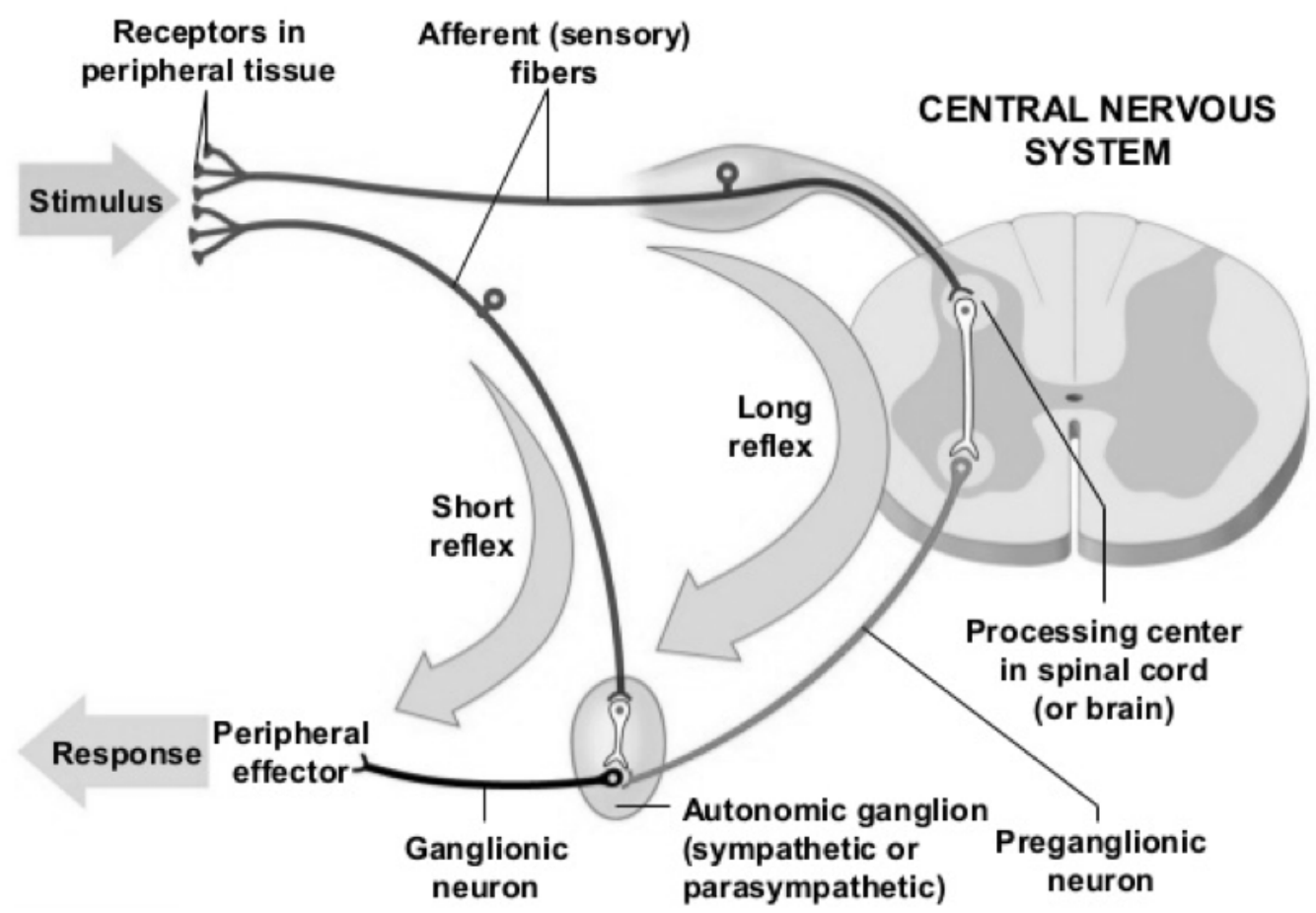

Figure 1.4. Long and short reflexes in sensory nervous system. In long-term reflexes, the sensory neurons send the information to the CNS where is processed and then send it to the peripheral effector. In short reflexes, the sensory neurons synapse directly with a peripheral effector generating the response.

\subsubsection{Role of sensory nervous system in vascular function}

Sensory neurons have a physiological role in maintaining vascular homeostasis [22]. Although CGRP does not play a primary role in the regulation of basal blood pressure in normal individuals $[23,24]$, it is suggested to have protective roles that are important for physiological and pathological conditions in the vascular system [25]. These include attenuation of VSM proliferation [26], hyperplasia [27, 28] and stimulation of endothelial cell proliferation and endothelial progenitor cells [29].

The SPNS is involved in some neurovascular syndromes such as migraine. Several studies have reported that SP and CGRP are released in this neuronal disorder [30] leading to a robust dilation of cranial blood vessels [31]. Moreover, application of CGRP receptor antagonist in acute migraine has been shown to attenuate CGRP-induced dilation of human isolated coronary arteries [32, 33]. 
Increased levels of CGRP have been also reported in cardiac failure and hypertension. However, there is evidence that an increase of CGRP is beneficial in preventing cardiovascular diseases associated with hypertension [22]. In fact, mice lacking $\alpha$-CGRP develop significant hypertension, suggesting a protective role of the neurotransmitter in the vascular disease [34].

\subsection{The vascular tone}

The vascular tone is determined by the contractile activity of VSMC in the walls of resistance arteries, which regulates the caliber of the vessel, and hence blood flow. According to Poiseuille's law, resistance is directly proportional to fluid viscosity $\mathrm{p}$ and tube length $L$ and is inversely proportional to vessel radius. Thus, small changes in the diameter of these small vessels lead to dramatic changes in resistance (Eq. 1.1).

$$
R=\frac{8 \rho L}{\pi r^{4}}
$$

Equation 1. Poiseuille's law, where $R$ is resistance to flow, $p$ refers to the viscosity, $L$ is the length and $r$ corresponds to the radius of the artery.

The regulation of the contractile state of VSMC is dependent on a complex interplay of vasodilator and vasoconstrictor stimuli from intrinsic and extrinsic processes, which implicates the circulation of hormones, endothelial secretions, neurotransmitter release and the modulation of physical factors such as the blood flow and the tension of the vessel wall [35]. All these signals are integrated by VSMC to determine the degree of contraction and hence the diameter of the blood vessel.

Among the intrinsic mechanisms, the myogenic response is a fundamental process for the development of resting vascular tone, allowing a constant blood flow despite changes in arterial pressure. This phenomenon is mediated by depolarization attributed to activation of non-selective cationic channels increasing the intracellular $\mathrm{Ca}^{2+}$ that would finally activate voltage-dependent $\mathrm{Ca}^{2+}$ channels (VDCC), leading to the contraction of the artery. Other important intrinsic factors are the endothelial secretions such as the vasoconstrictor endothelin and the vasodilator NO [1].

On the other hand, the extrinsic mechanism is also a fundamental control system of the TPR and is mainly regulated by endocrine factors and the perivascular nerves that 
innervate the resistance arteries (Figure 1.5). Non-selective channels present in both sympathetic and sensory perivascular nerves would be activated by external stimuli leading to an increase in cytosolic $\mathrm{Ca}^{2+}$ concentration within the neuron and consequently the release of NTs. These NTs can in turn activate their receptors on the VSMC and endothelial membrane resulting in the contraction or dilation of the blood vessel.

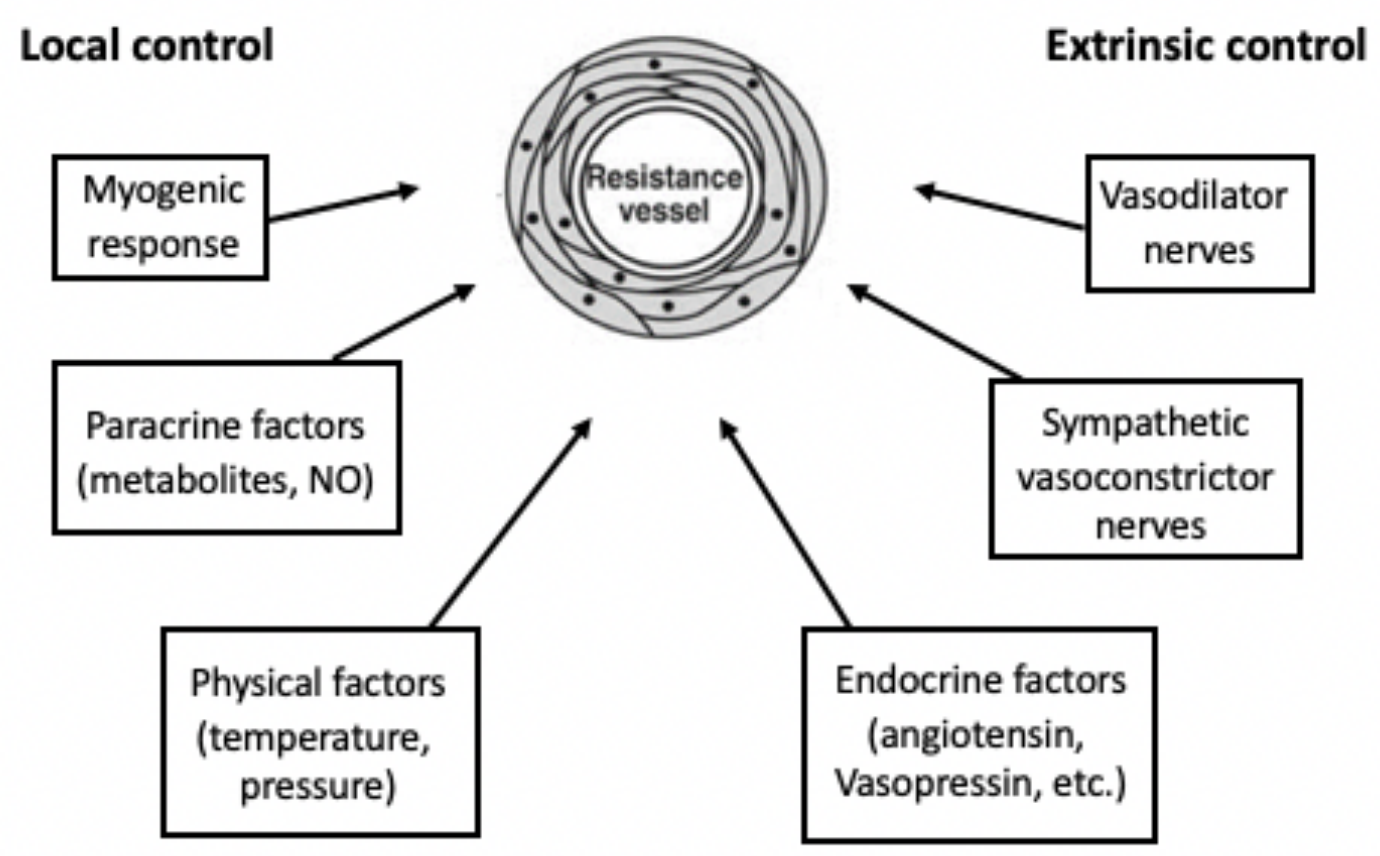

Figure 1.5. Overview of vascular control. Vascular tone is determined by many different competing vasoconstrictor and vasodilator influences acting on the blood vessel (modified from Levick J.R., 2003)

Among the non-selective channels, Transient Receptor Potential (TRP) channels have been proposed as molecular sensors involved in different vascular functions in response to diverse external stimuli playing important roles in the control of the TPR [36-40]. TRP channels are not only expressed in VSMC [41] but also in the perivascular nerves, where they contribute to cellular excitability [41-44]. 


\subsection{Transient Receptor Potential channels}

The TRP proteins constitute a large family of non-selective channels permeable to monovalent and divalent cations [45]. They are related to the product of the transient receptor potential (trp) gene in the fruit fly Drosophila melanogaster, where the founding member was discovered $[46,47]$. The 28 mammalian TRP proteins are split into six subfamilies according to their amino acid sequence homology: TRPC1-7 (Canonical), TRPM1-8 (Melastatin), TRPV1-6 (Vanilloid), TRPA1 (Ankyrin), TRPML1-3 (MucoLipin), and TRPP2,3 and 5 (Polycystin) [48, 49] (Figure 1.6).

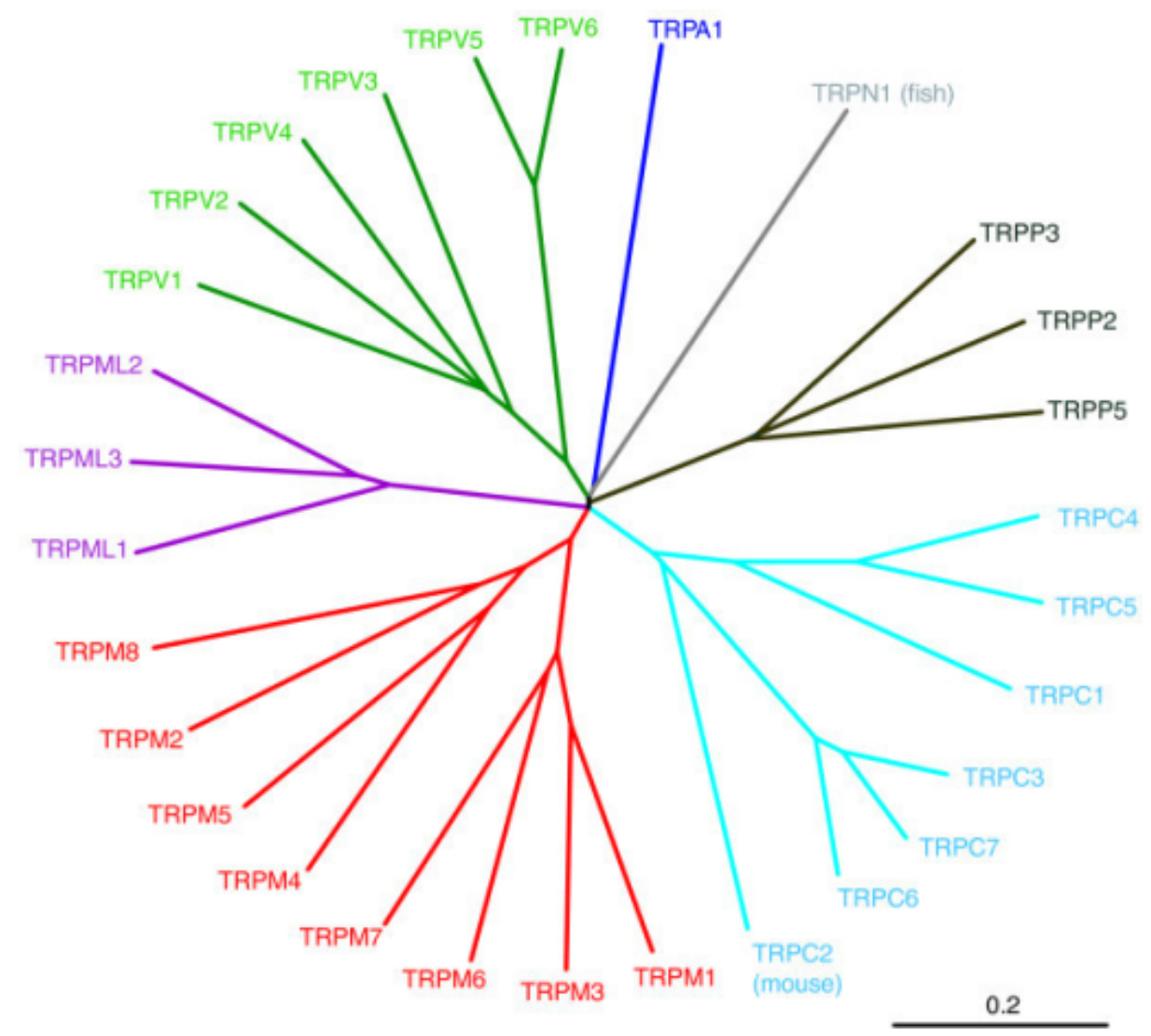

Figure 1.6. Phylogenetic tree of the TRP family of cation channels showing the classification into six subfamilies: TRPA (Ankyrin), TRPP (Polycystin), TRPM (Melastatin), TRPC (Canonical), TRPML (MucoLipin) and TRPV (Vanilloid) (Nillius, B. et al, 2011)

Until now, only the structures of TRPA1, TRPC4, TRPC3, TRPC6, TRPM2, TRPM4, TRPM7, TRPM8 (Figure 1.7 B), TRPV1, TRPV2, TRPV4 and TRPV6 channels have been determined by Cryo-electron microscopy [50-53]. All TRP channels, comprise a homo or heterotetramer of TRP proteins, each with six putative transmembrane segments with 
a loop between S5 and S6 forming the pore of the channel $[54,55]$. Intracellular amino and carboxyl termini are variable in length. The presence of ankyrin repeats, lipidinteractions domains, EF hands and phosporylation sites in the amino termini can influence channel gating $[56,57]$. The carboxyl termini are very variable amongst the different TRP protein containing calmodulin binding sites and entire enzymatic subunits $[58,59]$ (Figure 1.7A).

A

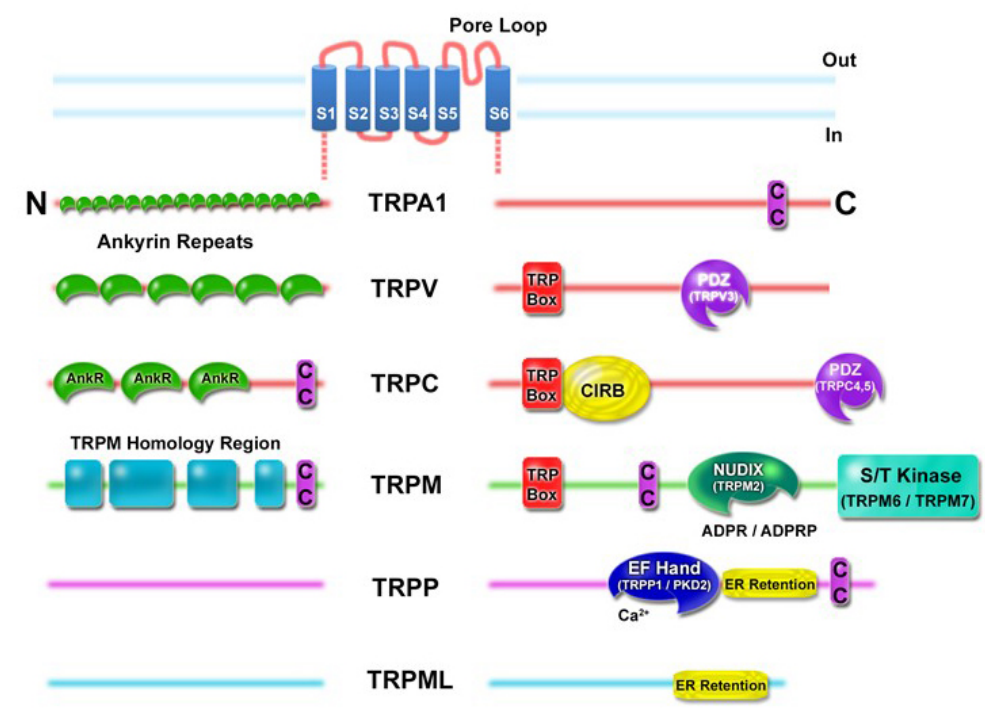

B
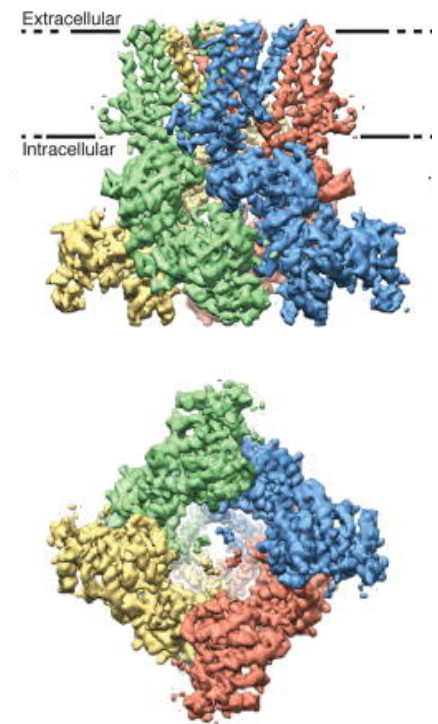

Figure 1.7. Topological structure of TRP channels and Cryo-EM reconstruction of TRPM 8 channel. (A) TRP channel monomer have six transmembrane topology (S1-S6) with a pore loop between $\mathrm{S} 5$ and $\mathrm{S} 6$. The cytoplasmic $\mathrm{N}$ - and $\mathrm{C}$ - termini contain different structural and functional elements as indicated for each subfamily. Some of the sensory TRP channels such as TRPA1 and TRPV family contain multiple ankyrin repeats at their amino termini while TRPM family contain a homology región (modified from Clapham, D.E., 2003). (B) Cryo-EM reconstruction of the TRPM8 channel (Yin et al., 2018).

TRP channels are ubiquitously expressed in a variety of tissues and mammalian cells and most of them are localized in the plasma membrane [59]. These channels can be activated by multiple stimuli ranging from endogenous and exogenous chemicals to physical stimuli such as membrane deformation and changes in membrane potential and temperature. The activation of these channels leads to the pore opening allowing a cation influx and depolarization of the cell at the resting potential triggering the activation of VDCC and resulting in $\mathrm{Ca}^{2+}$ entry to the cell $[49,60]$. The increase of free cytosolic $\mathrm{Ca}^{2+}$ concentration by TRP activation can modulate different downstream 
cellular mechanisms [59]. For instance, TRP proteins are essential for physiological processes including $\mathrm{Ca}^{2+}$ and $\mathrm{Mg}^{2+}$ homeostasis, the regulation of smooth muscle contraction, cell proliferation, migration, thermo-, chemo- and mechano-sensation processes and remodelling in pathological conditions [61-66].

Several members of the TRP protein family including TRPA1, TRPM3, TRPM8, TRPV1, TRPV2, TRPV3 and TRPV4 have been identified as sensory TRP channels due to their chemo-, thermo- and mechano-sensory properties and reported to be expressed mainly in sensory nerve fibres [67]. They play important roles in neuropeptide exocytosis [68], vascular physiology [69], pain sensation [70, 71], inflammatory processes [72, 73] and thermoregulation $[62,74,75]$.

\subsection{Sensory TRP channels in the vascular system}

TRPA1, TRPV1, TRPM3, TRPM8 and other TRP channels have been proposed as molecular sensors modulating vascular functions [76-80]. This thesis focuses in the arterial function of several sensory TRP cation channels in responses to external chemical and thermal stimuli.

Sensory TRP channels are involved in a variety of vascular functions, not only in modulation of VSMC contraction, but also in myogenic responses and $\mathrm{Ca}^{2+}$-induced VSMC proliferation and migration $[81,82]$. Although they have been identified in the vascular system, where they are expressed in endothelium, VSMC and perivascular nerve endings innervating the arteries, there are substantial discrepancies about their vascular functions in response to external stimuli.

\subsubsection{TRPA1}

TRPA1 is the sole member of the mammalian TRPA family [83]. It is a voltage-dependent nonselective cation channel permeable to $\mathrm{Ca}^{2+}, \mathrm{Na}^{+}$and $\mathrm{K}^{+}$, and is considered as a sensor of mechanical, chemical and noxious thermal stimuli. TRPA1 can be activated by a wide range of endogenous pungent and external irritants that trigger inflammatory processes and pain [78, 84-86]. Noxious compounds such as acrolein [78], allyl isothiocyanate [77] and cinnamaldehyde [87] are able to activate TRPA1 through covalent modification of $\mathrm{N}$-terminal cysteines or lysines. TRPA1 is also activated by the reactive compounds hypochlorite, hydrogen peroxide and NO [88- 
90]. Several studies have shown that TRPA1 is activated by cold in a noxious range [87, 91-93] and that they coexpressed with nociceptors markers such as CGRP and SP [91]. Other reports did not find evidence for activation of TRPA1 by cold [77, 94, 95].

The role of TRPA1 in the vascular system is not well-understood yet. Deletion of the Trpa1 gene in mice had no effect on blood pressure under baseline conditions or in response to angiotensin II [96]. Vascular expression of Trpa1 is not well determined although it was demonstrated to be expressed in many different layers of the arteries $[91,97,98]$. TRPA1 present in the endothelium of cerebral arteries, is concentrated within myoendothelial junction sites, where its activation mediates endotheliumdependent SMC hyperpolarization and vasodilatation that requires the activity of $\mathrm{Ca}^{2+}$ activated $\mathrm{K}^{+}$channels $[99,100]$. TRPA1 is activated by lipid peroxidation metabolites generated by reactive oxygen species (ROS) and involved in the ROS signaling pathway that causes endothelium-dependent vasodilation [101].

Many studies have revealed that TRPA1 channels are expressed in sensory perivascular nerves $[100,102]$ where they mediate vasodilatation of peripheral arteries in response to chemical agonists through the release of CGRP [103]. Yanaga et al. demonstrated a cinnamaldehyde-induce dilation in precontrated aortic rings that was not abolished in endothelium-denuded samples [104], suggesting a TRPA1-independent effect of cinnamaldehyde [105].

\subsubsection{TRPV1}

TRPV1 is a polymodal channel that can be activated by a variety of chemical and physical stimuli [106] including capsaicin [107], resiniferatoxin [107], low pH [108], endocannabinoid lipids [108], heat [107] and membrane depolarization [109]. This channel can be sensitized by the activation of PKA and pro-inflammatory mediators such as histamine and chemokines [110]. TRPV1 is the first channel describe to be activated by heat. The burning sensation of capsaicin suggested that heat and capsaicin could act through the same molecular pathway. Interestingly, a study revealed that TRPV1 is mainly expressed in peptidergic primary sensory neurons and its activation resulted in warm temperature and capsaicin-induced activation [107]. In fact, cultured sensory neurons from TRPV1-deficient mice showed a lack of heat-induced currents upon 
warming up to $43{ }^{\circ} \mathrm{C}$, although higher heat-induced responses (>55 ${ }^{\circ} \mathrm{C}$ ) remained unchanged $[76,111]$.

TRPV1 is heterogeneously expressed across VSM of blood vessels [112] and is regulated at the level of the individual blood vessels: some blood vessels showed intense TRPV1 immunostaining, whereas nearby vessels were negative [112]. It has been reported that activation of TRPV1 expressed in rat aortic myocytes by capsaicin causes endotheliumindependent vasoconstriction [113]. This was supported by another study showing that capsaicin increased $\mathrm{Ca}^{2+}$ uptake of VSMC, resulting in vasoconstriction [114].

In contrast, numerous authors have demonstrated the involvement of TRPV1 channels in the regulation of vascular tone in an endothelium-dependent manner [115-117]. Yang, D. et al. showed that acute administration of capsaicin for 6 months enhanced the production of NO and endothelium-dependent relaxation in the isolated mesenteric arteries from normal mice, an effect that was absent in Trpv1 KO mice [118]. Furthermore, capsaicin also attenuated the contractile response of thoracic aortic rings preconstricted with NA [119]. Importantly, immunohistochemical analysis identified the expression of TRPV1, SP, and CGRP in sensory neurons innervating the resistance arteries where its activation can trigger vasodilation via CGRP release $[120,121]$.

\subsubsection{TRPM3}

The Trpm3 gene consist of 28 exons and possesses several alternative splice sites [122, 123], which results in different isoforms [124] whose functions are still largely unexplored. TRPM3 is a voltage-dependent, non-selective cation channel that can be activated by intracellular $\mathrm{Mg}^{2+}[123]$ and blocked by $\mathrm{La}^{3+}$ [124]. Stimulation of TRPM3 typically induces outwardly rectifying currents and the single-channel conductance of the human TRPM3 is 65 pS for isotonic $\mathrm{Ca}^{2+}$ [125]. TRPM3 is reversibly activated by hypotonic cell swelling [125], oxidative stress and exposure to heat [62, 126].

Sphingolipids produced by the human body were the first TRPM3 activators to be described [127]. Another potent TRPM3 activator is the neurosteroid pregnenolone sulfate (PS) which is able to induces rapid and reversible TRPM3 activation, in overexpression systems and in cells endogenously expressing TRPM3 [128-131]. PS is produced in considerable amounts by the human body ([PS] in plasma $\approx 100-800 \mathrm{nM}$ ), although the conditions under which elevated PS levels may modulate TRPM3 remain 
unknown [132]. TRPM3 channels are expressed in mouse nociceptive neurons. Interestingly, in Trpm3-deficient mice, the number of PS-sensitive neurons is reduced. Intraplantar injections of PS in the paw induced nocifensive behavioral responses that were lacking in Trpm3 knockout (KO) mice [62].

Other related substances such as dehydorepiandrosterone (DHEA), DHEA sulfate and pregnenolone also activates TRPM3 but with a low efficacy [130]. Furthermore, other steroidal analog of PS, dehydroepiandrosterone sulfate, has been identified to activate TRPM3 channels $[133,134]$.

Nifedipine and 1,4-dihydropyridine also activate TRPM3 channels [130]. It has been discussed that nifedipine and PS act on TRPM3 in different biding-sites causing a larger activation of TRPM3 channels when both molecules are applied [134]. Interestingly, a new synthetic ligand called CIM0216 caused a potent activation of TRPM3 [135].

On the other hand, the first TRPM3 inhibitors were troglitazone, pioglitazone and rosiglitazone [136]. Other non-selective inhibitors of TRPM3 are flufenamic acid, tolfenamic acid and meclofenamic acid [131]. Some of the most potent TRPM3 inhibitors described so far are natural compounds including liquiritigenin and isosakuranetin [137]. Other flavanones such as hesperetin, eriodictyol and naringenin inhibit TRPM3 currents in freshly isolated DRG neurons [129]. Application of these TRPM3 inhibitors reduced the sensitivity of mice to PS-induced pain. The deoxybezoin ononetin has been also identified as a potent TRPM3 blocker $[129,136]$.

TRPM3 channels can be partially inhibited by cholesterol and other steroids such as progesterone, pregnanolone, estradiol, dihydrotestosterone and $210 \mathrm{H}$-progesterone [128]. However, the physiological relevance of these inhibitors on endogenously expressed TRPM3 is not yet known. Currents mediated by TRPM3 are inhibited by several synthetic compounds such as the nonsteroidal diclofenac, the anticonvulsant primidone and by the polyclonal antibody TM3E3 [128, 138, 139]. Nonspecific compounds can also inhibit TRPM3 such as 2-APB [140] and the lanthanides $\mathrm{Gd}^{3+}$ and $\operatorname{La}^{3+}[125,127]$.

TRPM3 expressed in a subset of sensory neurons, is also involved in noxious heat sensing since these heat-sensitive neurons are reduced in Trpm3-deficient mice. Trpm3 KO mice exhibited deficits in their responses to noxious heat as it was demonstrated by 
prolonged reaction latencies in the tail immersion and hot plate assays and a reduced avoidance of warm areas in the thermal gradient and thermal preference tests [62].

Systematic expression analyses of human and mouse TRPM3 channels in different tissues by quantitative real time polymerase chain reaction ( $q P C R$ ) indicated a high level of Trpm3 mRNA in the brain, sensory neurons, kidney, and adipose tissue [62, 125, 141143]. In addition, there is abundance of Trpm 3 transcripts in other tissues such as sperm cells, pancreas, heart, bladder and blood vessels [97, 128, 144-146].

A study by Naylor et al. demonstrated that functional TRPM3 channels are expressed in VSMC of human saphenous veins and mouse aorta by using TRPM3-specific antibody and siRNA [147]. Moreover, another study from the same group indicated potential functional value of TRPM3 in proliferating and contraction in VSMC $[128,148]$.

\subsubsection{TRPM8}

TRPM8 is a non-selective cation channel expressed in sensory neurons [149]. It is well known to be activated by cold temperatures and by chemical compounds including menthol, icilin, and several inflammatory agents [63, 150-152]. A phosphatidylinositol 4,5-bisphosphate $\left(\mathrm{PIP}_{2}\right)$-related mechanism has been proposed for the activation of TRPM8. Activation of $\mathrm{Ca}^{2+}$-dependent phospholipase $\mathrm{C}(\mathrm{PLC})$ shifts the voltage dependence of TRPM8 channel curve to more positive potentials and reduces the channel sensitivity to ligands such as menthol [153].

Other stimuli, such as oxidative stress [154] and the synthetic agonists such as cryosim3 (1-diisopropylphosphorylnonane) selectively activates TRPM8 [155]. On the other hand, novel synthetic compounds have been reported to block TRPM8 channels, for instance RQ-00434739 [156], AMTB [157], KPR-2579 [158], as well as the mentholderivate compound menthoxypropanediol [159].

It is known that TRPM8 plays a role in regulation of the vascular tone since it can stimulate both vasoconstriction and vasodilation processes depending on the previous vasomotor tone of the blood vessel [160]. A single study showed that Trpm8 mRNA is present in rat aortic, mesenteric, tail and femoral arteries [160] and its activation by menthol led to dilatory responses. However, in some cases menthol caused contractions of endothelium-denuded aortic samples. The cooling sensation that menthol causes in the human skin has been associated with an increased blood flow in the skin [161]. In 
contrast, Sun et al. have found that activation of TRPM8 attenuated vasoconstriction of the mesenteric artery and lowered blood pressure via a RhoA/Rho kinase mechanism [162]. Therefore, the exact role of TRPM8 channels in the vascular system remains unclear.

\subsubsection{Other sensory TRP channels}

Other TRP channels such as TRPV2 have been proposed to play an important role in the stretch-activated cation currents and myogenic constriction in retinal arterioles [163]. Activation of TRPV3 channels seems to promote $\mathrm{Ca}^{2+}$ influx and elicit endotheliumdependent dilation of cerebral parenchymal arterioles [164]. In uterine radial arteries, TRPV3 caused an endothelium-independent $\mathrm{IK}_{\mathrm{Ca}}$-mediated dilation via the NO-PKG pathway [165]. Furthermore, carvacrol, a TRPV3 agonist found in oregan, elicits endothelium-dependent vasodilation [166].

TRPV4 channels have been implicated as mediators of $\mathrm{Ca}^{2+}$ influx in both EC and VSMC [167]. The activation of endothelial TRPV4 channels led to endothelium-dependent vasodilation. However, very little is known about the functional role of TRPV4 in the resistance vasculature or how these channels influence hemodynamic properties. Several studies have found that activation of TRPV4 channels produces vasodilation of VSMC [168]. The presence of TRPV4 in cerebral VSMC has been demonstrated by immunohistochemistry and RT-PCR [167]. Furthermore, super-resolution imaging reveals clustering of TRPV4 channels in arterial myocytes [169]. Using microspectrofluorimetry and patch-clamp, Ducret et al. found that serotonin induced activation of TRPV4-like current followed by a sustained $\mathrm{Ca}^{2+}$ entry resulting in an increased of VSM proliferation [170]. In addition, TRPV4 channels can also functionally interact with other proteins. For instance, activation of TRPV4-TRPC1-K $\mathrm{Ca}_{1} 1.1$ complex by epoxyeicosatrienoic acids in human internal mammary arteries induced VSM membrane hyperpolarization followed by relaxation [171]. 


\subsection{Modulation of vascular function by thermal stimuli: role of sensory TRP channels}

The regulation of the vasculature in response to thermal stimuli is a fundamental homeostatic function in animals that is critical for survival. In fact, the control of body temperature is the main factor regulating blood flow in the cutaneous circulation. The cutaneous circulation is a good example of extrinsic regulation, as the tone of cutaneous vessels is under the control of the sympathetic nerves. Under basal conditions, there is an increased sympathetic tone and a vasoconstriction of the skin vessels, that can be accentuated in response to a decrease in environmental temperature, as a mechanism to minimize heat loss through the skin. The blood vessels subsequently dilate to restore blood flow for protection and survival. This basic adaptive physiological mechanism of vasoconstriction followed by vasodilation was first described by Thomas Lewis [172].

Conversely, increases in either body temperature or external temperature lead to vasodilation by inhibiting the tonic activity of sympathetic nerves, allowing blood flow increased to the cutaneous territories as a mechanism to dissipate heat. So, the systemic response against changes in external temperature is mainly governed by peripheral nerves fibres connected to the CNS. This central system integrates temperature changes and generates efferent responses to adjust heat-exchange rates with the environment $[173,174]$. In addition, peripheral nerves are also capable to induce fast vasoconstrictor or vasodilator effect in the cutaneous circulation in response to temperature changes as a protective mechanism [37].

Often, body cooling raises sympathetic vasoconstrictor activity through reflexes initiated by low temperature [175]. However, mammals often respond to maintained cool temperatures with a recovery vasodilatation (the "paradoxical vasodilation" mentioned before) and this is associated with a healthy peripheral vasculature [176]. In fact, a loss of cold-induced reflex recovery, associated with vasodilatation, is a marker of peripheral vascular diseases such as Raynaud's disease [177].

The contribution of the sympathetic tone to the control of the vasodilator mechanisms in response to temperature changes in the cutaneous circulation is well established [178]. However, the molecular mechanisms behind these vascular reflexes are still unknown and even the thermosensitive components remain unclear. Since some non- 
selective cation channels of the TRP family expressed in sensory neurons and other cells work as thermosensors being activated by changes in ambient temperature (Figure 1.8), there is strong evidence suggesting that sensory TRP channels, including TRPA1, TRPM8, TRPV1 and other TRP channels are also relevant in the local vascular responses to thermal changes [179].

The molecular sensors responsible for the detection of cold are sill unclear. However, TRPA1 is a controversial candidate in the cold-induced vascular pathway [180]. This controversy has not been yet resolved by the use of Trpa1-deficient mice in behavioral studies. While some studies found reduced noxious cold sensing in Trpa1 KO mice [181$184]$ others showed normal responses $[78,185]$ or only reduced cold responses when TRPA1 sensitivity is enhanced by exogenous agonists [186]. In addition, one study demonstrated that human TRPA1 could act as a bidirectional thermosensor depending on the presence of ligands contributing to both heat and cold sensation [187]. Other studies using pharmacological blockade of TRPA1 in mice have shown that TRPA1 is not involved in mediating cold responses $[188,189]$.
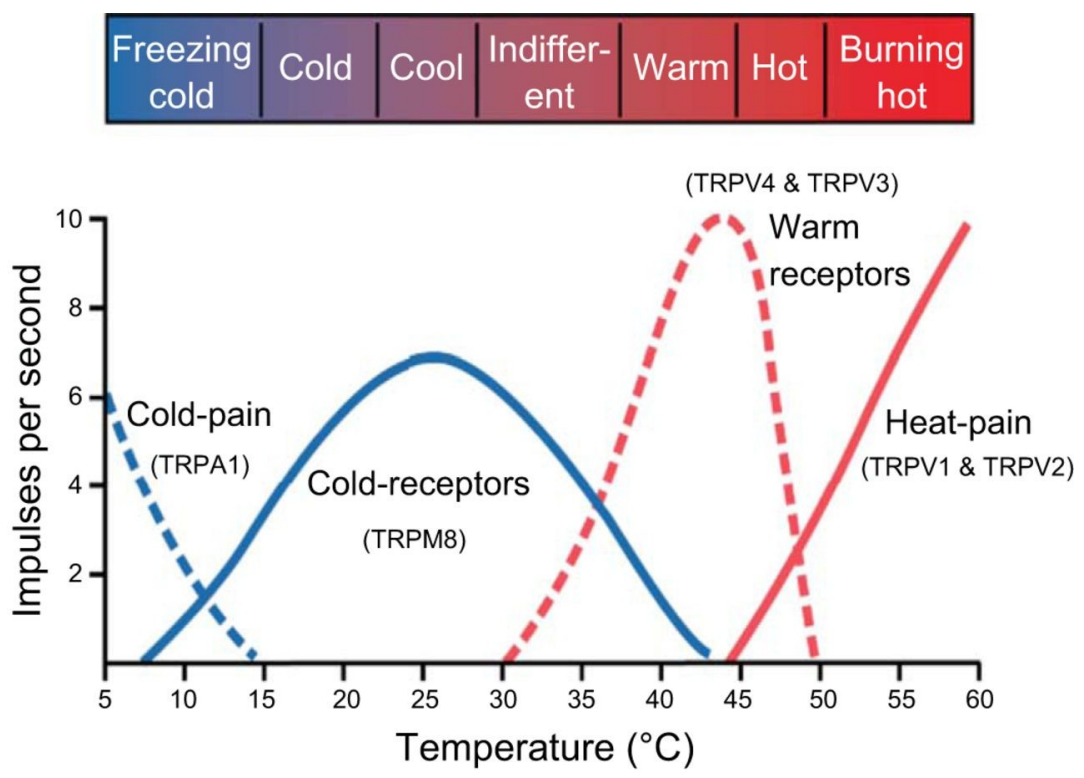

Figure 1.8. Frequencies at different skin temperatures of thermoreceptors, along with potential transient receptor potential (TRP) channels associated with receptor function (Tansey et al., 2015).

TRPA1 has also been proposed as a vascular sensor [37]. Cutaneous blood flow was reduced using cold water immersion of an anesthetized mouse paw. Similar vascular 
responses were found in wilt type mouse paw using a cold copper probe but not in Trpa1 $\mathrm{KO}$ mice. These results suggested that the two cold treatment techniques could produce TRPA1-dependent vascular responses via different mechanisms [37]. Furthermore, administration of TRPA1 antagonists significantly inhibited the vasoconstriction induced by the cold-water immersion demonstrating the relevance of TRPA1 in the vascular response. In accordance with this, using a full-field laser perfusion imager in vivo to measure cutaneous blood flow, Aubdool et al. found that TRPA1 is essential, not only in the initial vasoconstrictor response of the local cold-induced vascular response in the physiological reflex, but also in the restorative vasodilation [190].

Another candidate in the range of cold temperature is TRPM8, which can be activated by low temperatures. TRPM 8 rather than TRPA1 is considered to have a major role in the response to deep body cooling [191] and in cold-induced pain behaviors [192]. Furthermore, TRPM 8 channels are activated by compounds such as icilin and menthol that induce a cooling sensation [193]. Interestingly, intravenous injections of menthol made wild type rats to select warmer temperatures in a thermal gradient in order to maintain the core body temperature constant [194]. Several groups have demonstrated using Trpm8 KO mice, that TRPM8 are key channels responsible in the detection of external cold since the mutant animal did not respond to noxious cold $[150,152]$. In another study, it was found an increased latency for withdrawal from a cold plate in Trpm8-deficient mice compared to control mice [151]. In addition, menthol application also triggered autonomic responses such as shivering and tail skin vasoconstriction [195]. TRPM8 channels are known to be expressed in afferent neurons surrounding the vasculature where they can modulate vascular responses to external stimuli [160]. In fact, it was reported that cold-induced vasoconstriction was partially but significantly reduced in Trpm8 KO mice [190].

The physiologic response of vasoconstriction under localized cooling was prevented upon pharmacological blockade of TRPA1 and TRPM 8 channels suggesting that both channels are required to produce the cold-induced vascular response [37]. Using Trpa1/Trpm8 double KO mice, Pan et al. showed a large reduction in cold avoidance compared to that of single Trpm8 KO mice, suggesting that both receptors work together in the detection of the entire cold temperature range [37]. This further supports the findings of Winter and colleagues that both channels can influence the 
vascular cold response over the complete cold temperature range by the activity of coldactivated sensory fibers, resulting in a synergistic effect [196]. However, none of these studies explore the direct effect of thermal changes on the vasoconstrictor response of the blood vessel or the expression and the functional contribution of these channels to the cold-induced vascular responses.

In the warm temperature range, global Trpv1 KO mice have revealed that TRPV1 is involved in heat sensation [197]. Application of TRPV1 agonists such as capsaicin, which produces a hot sensation was found to decrease the basal temperature due to peripheral vasodilation [198]. In addition, capsaicin-induced ablation of sensory fibres and sensitized animals to heat challenge [199]. It has been reported that antagonist of TRPV1 induced vasoconstriction and modulated the thermoregulatory response [200]. Other study has demonstrated that TRPV1 and TRPV4 expressed in sensory nerves can modulate the vascular function under warm conditions [201].

Another TRP channel identified to be involved in noxious heat sensing is TRPM3 expressed in sensory neurons [62]. An increase in temperature to $37{ }^{\circ} \mathrm{C}$ caused a sensitization of TRPM3 to PS, and it has been postulated that even the physiological PS concentration found in the human body is more than sufficient to activate TRPM3 channels, especially at that temperature $[130,133]$. Importantly, inhibition of TRPM3 by flavanones reduced the sensitivity of mice to noxious heat [129]. TRPV2 channels have been reported to be expressed in sensory neurons representing as candidates for noxious heat sensing. Heterologous expression of TRPV2 channels produced a cation current at temperatures above $52{ }^{\circ} \mathrm{C}$ [202]. However, Trpv2 KO mice did not show any effect to noxious heat [203]. Whether TRPM3 and TRPV2 channels are involved in vascular responses to thermal challenges remains still unclear. 


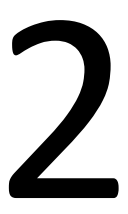

Hypothesis and Objectives 
The regulation of the arterial pressure under certain stimuli is an essential physiological process determined by the balance of competing vasoconstrictor and vasodilator influences acting on the resistance arteries. Recent studies indicate that sensory TRP channels expressed in the vascular system can be activated by a diverse range of stimuli and are able to influence cellular excitability and intracellular $\mathrm{Ca}^{2+}$ signaling resulting in their implication in the mechanisms underlying vascular tone regulation. However, their actual relevance in the vascular physiology in response to chemical and thermal challenges remains unknown.

Considering these precedents and the fact that sensory TRP channels are highly expressed along the vascular system, we hypothesized that sensory TRP channels activity may influence the arterial function and regulate the vascular tone of resistance arteries in response to chemical and thermal stimuli under physiological conditions. In order to address this hypothesis, we proposed the following aims:

1) To determine the expression pattern of transient receptor melastatin (TRPM) family in mesenteric arteries and particularly the functional localization of TRPM3 channels in the local control of vascular function by application of chemical stimuli. To address this objective, we performed qPCR, confocal imaging, $\mathrm{Ca}^{2+}$-imaging, pressure myography and patch clamp experiments in mesenteric arteries from c57bl/6j mice and Trpm3 KO mice as control.

2) To determine the possible contribution of TRPA1 and TRPM8 channels to the vascular responses to local cold in isolated cutaneous arteries. The role of both channels in cold-induced systemic reflexes (involving CNS regulation) has been already described by Aubdool et al. [190]. In our study, we investigate if isolated peripheral cutaneous arteries have an intrinsic cold-sensitivity and whether TRPA1 and TRPM8 channels contribute to this local response. For that, we performed pressure myography experiments in isolated plantar arteries from c57bl/6j, Trpa1 and Trpm8 KO mice to determine changes in diameter upon exposure to low temperature and to explore the contribution of cold-induced activation of these channels to the vascular responses. 
Materials and Methods 


\subsection{Animals}

The experiments were performed on c57bl/6j (Janvier Laboratories, Berthevin Cedex, France), Trpm8 wild type, Trpm3 KO (Lexicon genetics; MGI:3528836), Trpv4 KO, Trpa1 KO [182] and Trpm8 KO male mice on a c57bl/6j background (Jackson laboratories, Maine, USA) [150], weighing about $25 \mathrm{~g}$ and from 10 to 12 weeks of age. Mice were housed in a conventional facility on a $12 \mathrm{~h}$ light-dark cycle and they received standard food and drinking water ad libitum. Animals were anesthetized and then euthanized by $\mathrm{CO}_{2}$ inhalation. All protocols were in accordance with the European Community and Belgian Governmental guidelines for the use and care of experimental animals (2010/63/EU, CE Off Jn8L358, LA12110551) and approved by the KU Leuven Ethical Committee Laboratory Animals (ECD) and the Institutional Care and Use Committee of the University of Valladolid.

\subsection{Vascular smooth muscle cells isolation}

\subsubsection{Surgery}

Mice were anesthetized and then euthanized by $\mathrm{CO}_{2}$ inhalation. Plantar or mesenteric arteries were isolated from each mouse. Subsequently, all samples were carefully dissected and cleaned from adipose tissue in cold oxygenated smooth muscle dissociation solution (SMDS) containing (in mM): $145 \mathrm{NaCl}, 4.2 \mathrm{KCl}, 0.6 \mathrm{KH}_{2} \mathrm{PO}_{4}, 1.2$

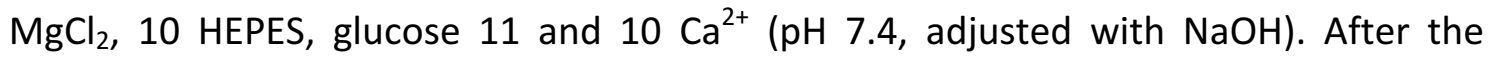
cleaning, they were processed in different ways depending on their final use: (1) the arteries were subjected to an enzymatic digestion to either get fresh dispersed VSMC or for further extract RNA or (2) directly used to carry out myography experiments and immunostaining assays.

\subsubsection{Enzymatic digestion}

The dissected arteries were cut into small pieces and subjected to two consecutive processes of enzymatic digestion. The first digestion was carried out at $37^{\circ} \mathrm{C}$ for $15 \mathrm{~min}$ in SMDS-Ca ${ }^{2+}$-free solution containing $0.8 \mathrm{mg} / \mathrm{ml}$ papain (Worthington Biochemical Corp.), $1 \mathrm{mg} / \mathrm{ml}$ Bovine Serum Albumin (BSA) (Sigma-Aldrich), and $1 \mathrm{mg} / \mathrm{ml} \mathrm{1,4-}$ Dithioerythritol (DTE) (Sigma-Aldrich). The second digestion was performed at $37^{\circ} \mathrm{C}$ for 
15 min using $10 \mu \mathrm{M} \mathrm{Ca}{ }^{2+}$ SMDS supplemented with $0.6 \mathrm{mg} / \mathrm{ml}$ collagenase $\mathrm{F}$ (SigmaAldrich), and $1 \mathrm{mg} / \mathrm{ml}$ bovine serum albumin (BSA) (Sigma-Aldrich). Digested arteries were rinsed twice with $10 \mu \mathrm{M} \mathrm{Ca}{ }^{2+}$ SMDS. After this washing step, single cells were obtained by mechanical disruption with a wide-bore glass pipette. Cells were maintained at $4{ }^{\circ} \mathrm{C}$ until they were used in patch-clamp recordings.

\subsection{Sympathetic ganglia isolation}

c57bl/6j male mice were anesthetized and then euthanized by $\mathrm{CO}_{2}$ inhalation. Cervical sympathetic ganglia were isolated from the sympathetic chain. Subsequently, they were carefully cleaned from adipose tissue in cold oxygenated SMDS containing $10 \mu \mathrm{M} \mathrm{Ca}^{2+}$. After the cleaning, the ganglia were subjected to RNA extraction procedure.

\subsection{RNA extraction and cDNA synthesis}

Arteries were enzymatically digested as described in section 3.2.2, but decreasing the incubation time to 9 min with the enzymes and skipping the washing step with SMDS$10 \mu \mathrm{M} \mathrm{Ca}^{2+}$, so that nerve fibres, smooth muscle and endothelial cells were still present in the preparation. Arteries of 6 mice were used for each determination. Total RNA from the digested arteries or sympathetic ganglia was extracted using RNeasy mini kit (Qiagen), following manufacturer's protocol. Once RNA was purified, quantification and verification of their quality was assessed using Experion RNA Stdsens Analysis kit (BioRad), samples with RNA quality indicator (RQI) values below 8 were discarded. cDNA synthesis was performed with 500 ng of total RNA using Ready-to-go First strand beads (GE Healthcare) and used for quantitative real-time polymerase chain reaction (qRTPCR).

\subsection{Quantitative real-time polymerase chain reaction}

A small fraction of the cDNA synthetized was used for qRT- PCR. qPCR reactions were performed with the 7500 Fast Real-Time PCR System (Life Technologies) by incubating at $50{ }^{\circ} \mathrm{C}$ for $2 \mathrm{~min}$ and $95^{\circ} \mathrm{C}$ for $10 \mathrm{~min}$, then $40 \mathrm{cycles}$ of $95^{\circ} \mathrm{C}$ for $15 \mathrm{~s}$ and $60{ }^{\circ} \mathrm{C}$ for 1 min. Each qPCR reaction $(20 \mu \mathrm{l})$ contained $3 \mu \mathrm{l}$ of cDNA template, $10 \mu \mathrm{l}$ of Universal TaqMan MasterMix (2x concentrated, Life Technologies), $1 \mu$ l of TaqMan assay (20x 
concentrated, Life Technologies) and $6 \mu \mathrm{l} \mathrm{H}_{2} \mathrm{O}$. For every experiment, test reactions were performed in triplicate, and non-template negative controls (NTC) in duplicate.

Fluorescent signals generated during PCR amplifications were normalized to an internal reference selected between two potential housekeeping genes $\beta$-actin and glyceraldehyde-3P-dehydrogenase (GAPDH) for accurate quantification of interested gene expression levels in each sample. We used GAPDH as endogenous gene for representing the data. The threshold cycle $(\mathrm{Ct})$ was set within the exponential phase, and the relative quantitative evaluation of target gene levels was performed using the $2^{-\Delta C t}$ method. Differences between samples with and without endothelium were calculated with the $2^{-\Delta \Delta \mathrm{Ct}}$ method, using the samples with endothelium as the calibrator $\left(\Delta \Delta \mathrm{Ct}=\Delta \mathrm{Ct}_{\text {without }}-\Delta \mathrm{C}_{\text {twith }}\right)$.

\subsection{Immunofluorescence microscopy}

Intact arteries from c57bl/6j and Trpm3 KO mice were fixed with 4\% paraformaldehyde (PFA) in phosphate-buffered saline (PBS) for $15 \mathrm{~min}$, permeabilized in PBTx (PBS, 0.2\% Triton X-100) and blocked with PBTx with $2 \%$ of sheep serum for $3 \mathrm{~h}$. Arteries were incubated overnight at $4{ }^{\circ} \mathrm{C}$ with the primary rabbit anti-TRPM3 (1:100, Santa Cruz), mouse anti-NF-200 (1:1000, MFCD02263444, sigma Aldrich), human PGP9.5 antibody (1:100, sc176636, Santa Cruz), chicken anti-beta-gal IgY (1:1000, ab9361, Abcam), rabbit anti calcitonin gene-related peptide (1:500, ab47027, Abcam), mouse anti-tyrosine hydroxylase (1:1000, ab129991, Abcam) or rabbit anti-alpha smooth muscle actin (1:250, ab5694, Abcam) antibodies, followed by the secondary antibodies Alexa 594 goat anti-rabbit (1:1000, A-11012, Molecular Probes), Alexa 555 goat anti-mouse (1:1000, ab150114, Abcam), Alexa 488 donkey anti-chicken (1:1000, ab63507, Abcam, Alexa 488 goat anti-mouse (1:1000, ab150117, Abcam) or goat anti-chicken IgY-Alexa 488 (1:1000, ab150173, Abcam). Secondary antibodies were prepared in blocking solution and incubated for $2 \mathrm{~h}$ at room temperature. Finally, the arteries were flatmounted in glass slides using DAPI-containing mounting solution (VectaShield, Vector Laboratories).

Human Embryonic Kidney 293 (HEK293T) cells were plated in poly L-lysine-coated coverslips, fixed with 4\% PFA in PBS for 15 min and blocked with PBS with $2 \%$ sheep 
serum for $3 \mathrm{~h}$. The cells were incubated with primary antibody rabbit anti-TRPM3 (1:100, Santa Cruz) overnight at $4{ }^{\circ} \mathrm{C}$. After that, samples were incubated with the secondary antibody Alexa 594 goat anti-rabbit (1:1000, Molecular Probes) in blocking solution during $2 \mathrm{~h}$ at $22^{\circ} \mathrm{C}$. The coverslips were mounted using DAPI-containing mounting solution (VectaShield, Vector Laboratories).

Confocal images of labelled cells and arteries were collected using the optimal pinhole size for the Plan-Apochromat 63x/1.4 oil objective and 20x or 40x objective of a Zeiss LSM 510 Meta Multiphoton microscope (Carl Zeiss AG). Images were acquired by consecutive excitation with Argon laser at $488 \mathrm{~nm}$ and He-Ne laser at $543 \mathrm{~nm}$. For nuclear DAPI staining, we used a two-photon pulsed excitation by the Spectra-Physics (Mountain View) Mai Tai laser at $770 \mathrm{~nm}$. Images were analyzed using ImageJ processing software. The z-position in the single plane images was determined after ad-hoc live zscanning of nuclei, according to the clear morphological differences between adventitia (round nuclei) and VSMC nuclei (elongated, perpendicular to the longitudinal axis of the artery, and below the round nuclei).

\subsection{Pressure myography experiments}

Third order mesenteric and plantar arteries ( $200 \mu \mathrm{m})$ were dissected and mounted in a myograph (Danish Myo Technology 110P) that allowed controlling the luminal pressure while measuring external arterial diameter via digital video edge detection (CCD camera). Arterial segments were cannulated between two borosilicate glass pipettes and fixed with nylon filaments at both ends. The artery segments were filled with physiological saline solution containing $(\mathrm{mM}): 120 \mathrm{NaCl}, 2.5 \mathrm{CaCl}_{2}, 1.17 \mathrm{MgSO}_{4}, 5$ $\mathrm{KCl}, 1.18 \mathrm{Na}_{2} \mathrm{HPO}_{4}, 25 \mathrm{NaHCO}_{3}, 1$ EDTA, 10 glucose $\left(\mathrm{pH} 7.4\right.$ adjusted with 5\% $\mathrm{CO}_{2}-95 \%$ air, which was maintained throughout the duration of the experiment). Arteries were pressurized to $70 \mathrm{mmHg}$ and allowed to stabilize at $37{ }^{\circ} \mathrm{C}$ for at least 15 min before starting the measurements. Unless otherwise stated the artery segments were airbubbled though the lumen to remove endothelial cells. Phenylephrine (10 $\mu \mathrm{M})$ or NA $(20 \mu \mathrm{M})$ were perfused to contract the arteries prior to the application of test compounds. PS and CIM were washed after their last application with physiological saline solution containing $10 \mu \mathrm{M}$ phenylephrine. The data were analyzed using MyoView 
software. At the end of each experiment, we applied the L-type $\mathrm{Ca}^{2+}$ channel blocker nifepidine $(10 \mu \mathrm{M})$ to determine the maximum arterial diameter. Vasodilation was determined using the formula:

$$
\text { Vasodilation }(\text { in } \%)=\left(100 *\left(\frac{D x-D P h e}{D N i f-D P h e}\right)\right)
$$

Dx, DPhe and DNif are the diameters recorded in the presence of test compound plus phenylephrine, phenylephrine alone and nifedipine, respectively. The resulting doseresponse curves for the test compounds $(X)$ were fitted using either one or two Hill functions of the forms:

$$
\text { Vasodilation }(\text { in } \%)=100 \frac{[X]^{n}}{[X]^{n}+k^{n}}
$$

were $[\mathrm{X}], \mathrm{n}$ and $\mathrm{k}$ are the test compound concentration, the Hill coefficient and the effective concentration, respectively, and:

$$
\text { Vasodilation }(\text { in } \%)=100\left(\frac{A_{1}[X]^{n 1}}{[X]^{n 1}+k_{1}^{n 1}}+\frac{\left(1-A_{1}\right)[X]^{n 2}}{[X]^{n 2}+k_{2}^{n 2}}\right)
$$

were $A_{1}, n 1$ and $k_{1}$ are the relative amplitude, the Hill coefficient and the effective concentration of the first Hill component, respectively, and $n 2$ and $k_{2}$ are the Hill coefficient and the effective concentration of the second Hill component, respectively. In the cold stimulus, vasoconstriction was determined using the formula:

$$
\text { Vasoconstriction }(\text { in } \%)=-\left(100 *\left(\frac{D x-D P h e}{D N i f-D P h e}\right)\right)
$$

Dx, DPhe and DNif were the diameters recorded in the presence of cold stimulus and phenylephrine, phenylephrine alone and nifedipine, respectively. A maximum of two arteries was taken from each mouse. In those cases, the data were averaged. 


\subsection{Intracellular $\mathrm{Ca}^{2+}$ fluorescence imaging}

$\mathrm{Ca}^{2+}$ imaging experiments were conducted with the fluorescent indicator Fura-2AM. HEK293T cells stably expressing murine TRPM3 were cultured as previously described [62]. They were incubated with $5 \mu \mathrm{M}$ Fura-2AM (Invitrogen) for $30 \mathrm{~min}$ at $37^{\circ} \mathrm{C}$. Fluorescence measurements were performed with a Zeiss Axioskop FS upright microscope fitted with an ORCA ER charge-coupled device camera. Fura-2AM was excited at 340 and $380 \mathrm{~nm}$ with a rapid switching monochromator (TILL Photonics). Mean fluorescence intensity ratios (F340/F380) were displayed online with Metafluor software (Molecular Devices). The standard extracellular solution contained (in $\mathrm{mM}$ ) $140 \mathrm{NaCl}, 3 \mathrm{KCl}, 2.4 \mathrm{CaCl}_{2}, 1.3 \mathrm{MgCl}_{2}, 10$ HEPES and 10 glucose (pH7.4 adjusted with $\mathrm{NaOH})$.

\subsection{Patch-clamp electrophysiology}

Whole-cell patch-clamp recordings were performed in freshly isolated VSMC at $22{ }^{\circ} \mathrm{C}$ using an Axopatch 200 patch-clamp amplifier (Axon Instruments, Molecular Devices), filtered at $2 \mathrm{kHz}$ (-3 dB, four-pole Bessel filter), and sampling at $10 \mathrm{kHz}$. Recordings were digitized with a Digidata 1322A interface, driven by CLAMPEX 10 (Axon Instruments). Patch pipettes were made from borosilicate glass ( $2.0 \mathrm{~mm}$ O.D., WPI) and double pulled (Narishige PP-83) to resistances ranging from 2 to $5 \mathrm{M} \Omega$ when filled with the internal solution. For $\mathrm{K}_{\mathrm{v}}$ channel recordings, the composition of this solution was (in $\mathrm{mM}$ ): 125 $\mathrm{KCl}, 4 \mathrm{MgCl}_{2}, 10$ Hepes, 10 EGTA, $5 \mathrm{Mg}^{2+}$-ATP, pH 7.2 adjusted with $\mathrm{KOH}$. The composition of the bath solution was (in $\mathrm{mM}$ ): $141 \mathrm{NaCl}, 4.7 \mathrm{KCl}, 1.2 \mathrm{MgCl}_{2}, 1.8 \mathrm{CaCl}_{2}$, 10 glucose, 10 Hepes and $500 \mathrm{nM}$ paxilline, $\mathrm{pH} 7.4$ adjusted with $\mathrm{NaOH}$. The voltage dependence of total $\mathrm{K}^{+}$current was obtained applying $200 \mathrm{~ms}$ pulses from a holding potential of $-80 \mathrm{mV}$ to voltages between -60 to $+60 \mathrm{mV}$ in $20 \mathrm{mV}$ steps at $5 \mathrm{~s}$ intervals. Whole-cell patch-clamp recordings of TRP currents were carried out with solutions of the following composition (in $\mathrm{mM}$ ): $141 \mathrm{NaCl}, 1.8 \mathrm{CaCl}_{2}, 1.2 \mathrm{MgCl}_{2}, 5 \mathrm{CsCl}, 10$ glucose, 10 HEPES, 0.005 nicardipine (a voltage-dependent $\mathrm{Ca}^{2+}$ channel antagonist), 0.1 DIDS (an anion exchange inhibitor) and 0.1 niflumic acid (chloride channel inhibitor), pH 7.4 adjusted with $\mathrm{NaOH}$ for the extracellular solution and $10 \mathrm{CsCl}, 110 \mathrm{Cs}$ aspartate, $10 \mathrm{NaCl}$,

3.2 $\mathrm{CaCl}_{2}, 10$ HEPES, 10 BAPTA, $2 \mathrm{Mg}^{2+}$-ATP ( $\mathrm{pH} 7.2$, adjusted with $\mathrm{CsOH}$ ) and with an 
estimated free $\left[\mathrm{Ca}^{2+}\right]$ of $100 \mathrm{nM}$ for the intracellular solution. To determine the effects of PS (10 $\mu \mathrm{M}$ and $30 \mu \mathrm{M})$, CIM0216 $(2 \mu \mathrm{M})$ and Uridine-5'-triphosphate (UTP, $100 \mu \mathrm{M})$ currents were recording upon stimulation with $1 \mathrm{~s}$ voltage ramps from $-150 \mathrm{mV}$ to +80 $\mathrm{mV}$ applied from a holding potential of $-60 \mathrm{mV}$ at $5 \mathrm{~s}$ intervals. Electrophysiological data analyses were performed with the Clampfit subroutine of pCLAMP (Axon Instruments) and with Origin 9 software (OriginLab Corp.).

\subsection{Reagents}

Pregnenolone sulfate (PS), phenylephrine, AngIl, nifedipine, guanethidine and UTP were purchased from Sigma-Aldrich. CIM0216 was obtained from Prof. Joris Vriens (Department of Development and Regeneration, KU Leuven). The CGRP receptor antagonist BIBN 4096 was obtained from Tocris Bioscience. $K_{V}$ channel toxin blockers, paxilline and stromatoxin, were dissolved in dimethyl sulfoxide (DMSO) and purchased from Alomone Laboratories. Correolide was a gift from María García (Merck Research Laboratories, New Jersey). The TRPA1 blocker HC030031 was purchased from Tocris Bioscience. DMSO was also used as vehicle for PS, nifedipine, BIBN, CIM0216 and $\mathrm{HC} 030031$. Angll was dissolved in saline solution (0.9\% $\mathrm{NaCl})$ and Milli- $\mathrm{Q}^{\circledR}$ purified water was used as vehicle for guanethidine, phenylephrine, UTP and potassium blockers.

\subsection{Statistical analysis}

In all experiments, data were pooled from multiple trials carried out on cells or arteries isolated from at least three different animals and summarized as means \pm SEM and dots plots for each individual measurement. The Origin software (version 9, OriginLab) was used for statistical analysis and data display. Differences between means were assessed using t-test paired or unpaired and one-way ANOVA, Dunn-Sidak test comparisons. ${ }^{*} \mathrm{P}$ $<0.05$; $P<0.01$ were taken as statistically significant difference between means and $n$ denotes the sample size. 


\title{
4
}

\section{Activation of the cation channel TRPM3 in perivascular nerves induces vasodilation of resistance arteries}

\author{
Lucía Alonso-Carbajo ${ }^{1,2}$, Yeranddy A. Alpizar ${ }^{1}$, Justyna B. Startek ${ }^{1}$, José Ramón López-López ${ }^{2}$, \\ María Teresa Pérez-García ${ }^{2}$ and Karel Talavera ${ }^{1}$ \\ ${ }^{1}$ Department of Cellular and Molecular Medicine, Laboratory of Ion Channel Research, KU Leuven; VIB Center \\ for Brain \& Disease Research, Leuven Belgium. \\ 2 Departamento de Bioquímica y Biología Molecular y Fisiología, Instituto de Biología y Genética Molecular, \\ Universidad de Valladolid y CSIC, Valladolid, Spain.
}

The results in this chapter were integrally published in Journal of Molecular and Cellular Cardiology. Lucía Alonso-Carbajo performed the majority of the experiments and analyzed the data. The manuscript was written by her with input from the other authors. 
Recent findings unveiling TRPM3 as potential chemo-sensor in nociceptive neurons [62] suggest that, if present in perivascular nerve endings, activation of this channel might result in neuropeptide release. This would suppose a vasodilating effect of TRPM3 activation, an action opposite to the vasoconstriction previously described in mouse aorta [128]. In order to address this question, we investigated the localization, function and mechanism underlying of TRPM3 in mouse mesenteric arteries.

\subsection{Expression pattern of TRPM family in mouse mesenteric arteries}

We first determined the expression of the genes encoding TRPM channels in c57bl/6j mouse mesenteric arteries with and without endothelium. Trpm1, Trpm5 and Trpm8 could not be detected after 40 cycles of amplification in any preparation. In contrast, the other 5 members of the subfamily were detected in preparations with endothelium (Figure 4.1A). We found lower relative expression for Trpm4 and Trpm7 and no detectable Trpm2 and Trpm6 in preparations devoid of endothelium (Figure 4.1A and B), suggesting for a preferential expression of these transcripts in the endothelial layer.

A

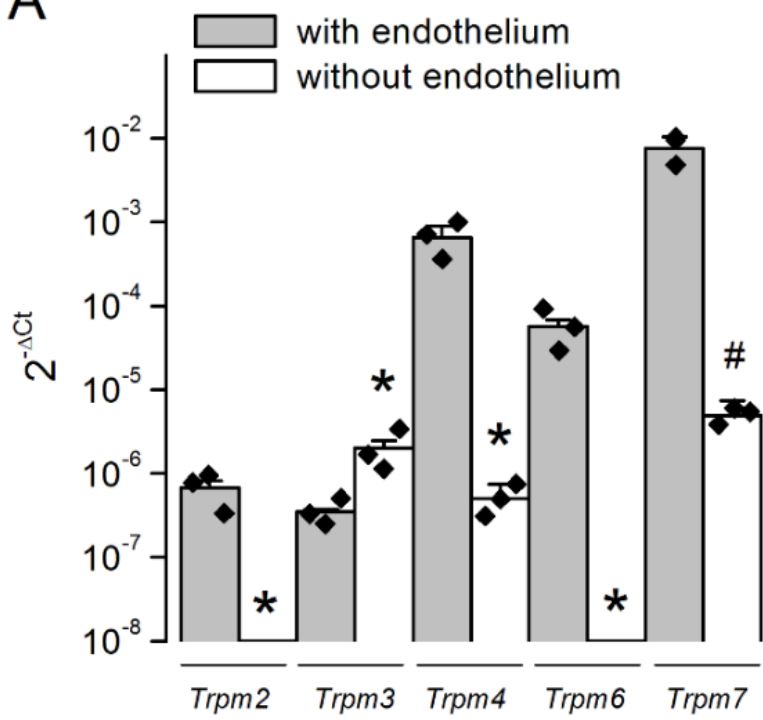

B

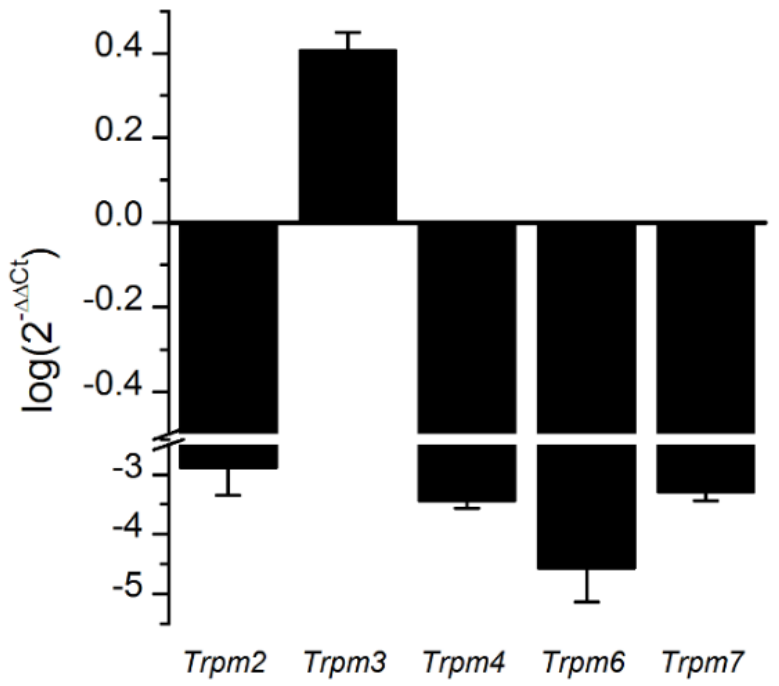

Figure 4.1. Expression of TRPM family in mesenteric arteries with and without endothelium. $(A)$ Relative expression of Trpm genes in mesenteric arteries dissected from c57bl/6j mice. (B) Relative expression of Trpm genes in endothelium-denuded arteries using arteries with endothelium as calibrator. Data are represented as mean \pm SEM, ${ }^{*} P<0.05 ;{ }^{\#} P<0.01(n=3)$, one way ANOVA, DunnSidak test.

In sharp contrast, we found higher relative levels of Trpm3 mRNA in endothelium-free preparations, indicating for a predominant expression in the medial and/or adventitial layers. 
Of note, we found a much higher relative abundance of Trpm3 mRNA in aorta than in mesenteric arteries $(3.3 \pm 0.8 ; \mathrm{n}=3$ versus $0.09 \pm 0.01 ; \mathrm{n}=3 ; P<0.001)$.

\subsection{Localization of TRPM3 in perivascular nerves of mesenteric arteries}

Confocal images of mesenteric arteries labeled with the neuronal marker PGP9.5 (Figure 4.2A) evidenced the presence of nerve fibres in the adventitial layer.

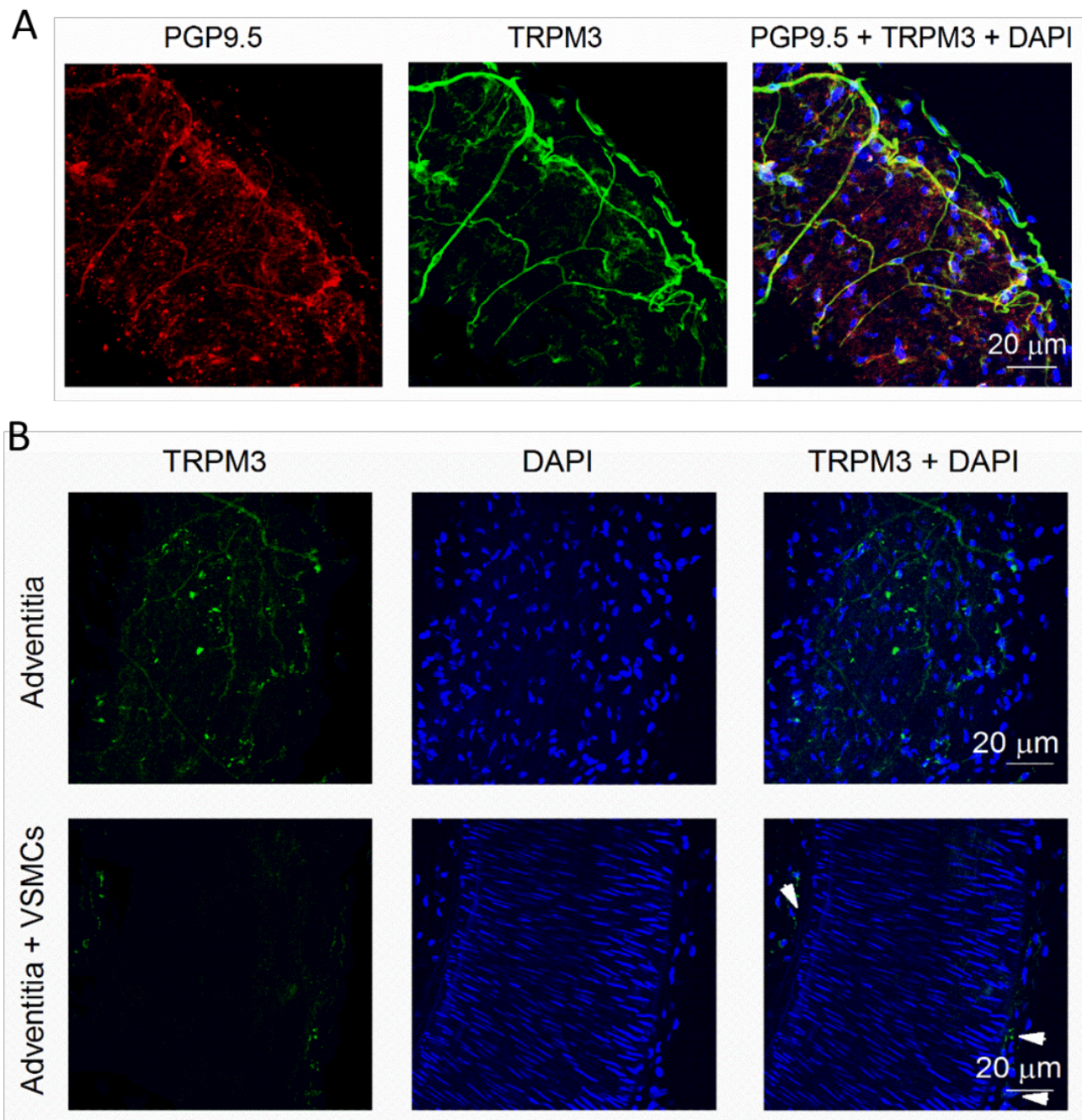

Figure 4.2. TRPM 3 is located in perivascular nerve endings of mouse mesenteric arteries. (A) Z-stack confocal images of intact c57bl/6j mouse mesenteric arteries labelled with PGP9.5 (red, left) and TRPM3 (green, center) antibodies. An overlay of these images merged with a nuclear staining DAPI (blue) is shown on the right. The initial position was set where no nuclei are visible and the steps have $1.03 \mu \mathrm{m}$ as suggested by the software. The image shows a $35.11 \mu \mathrm{m}$ thickness, acquired at $4.12 \mu \mathrm{m}$ from the initial position, 40X. The images are the sum of 16 readings average $(B)$ Confocal microscopy images of the adventitia and medial layers labelled with a TRPM3 antibody (green) and nuclear DAPI staining (blue). The white arrowheads in the bottom-right image point to TRPM3 labelling observed only in the adventitial layer. The images were taken by repositioning according to nuclei morphology. Images are representative of at least 3 independent experiments. 
We found that TRPM3 colocalized with PGP9.5 (Figure 4.2A) and was absent in cells of the tunica media (VSMC), which were clearly identified by the distinct orientation of their nuclei, perpendicular to the axis of the vessel (Figure 4.2B).

The localization of TRPM3 was determined with an antibody whose specificity against TRPM3 was confirmed in a HEK293T cell line stably transfected with this channel (Figure 4.3). As a control for the experiment, we used TRPM5-transfected HEK293T cells, which also express TRPM7 endogenously. We found that these cells were not stained with the anti-TRPM3 antibody, confirming the specificity for TRPM3 versus these closely-related TRPM channels (Figure 4.3).

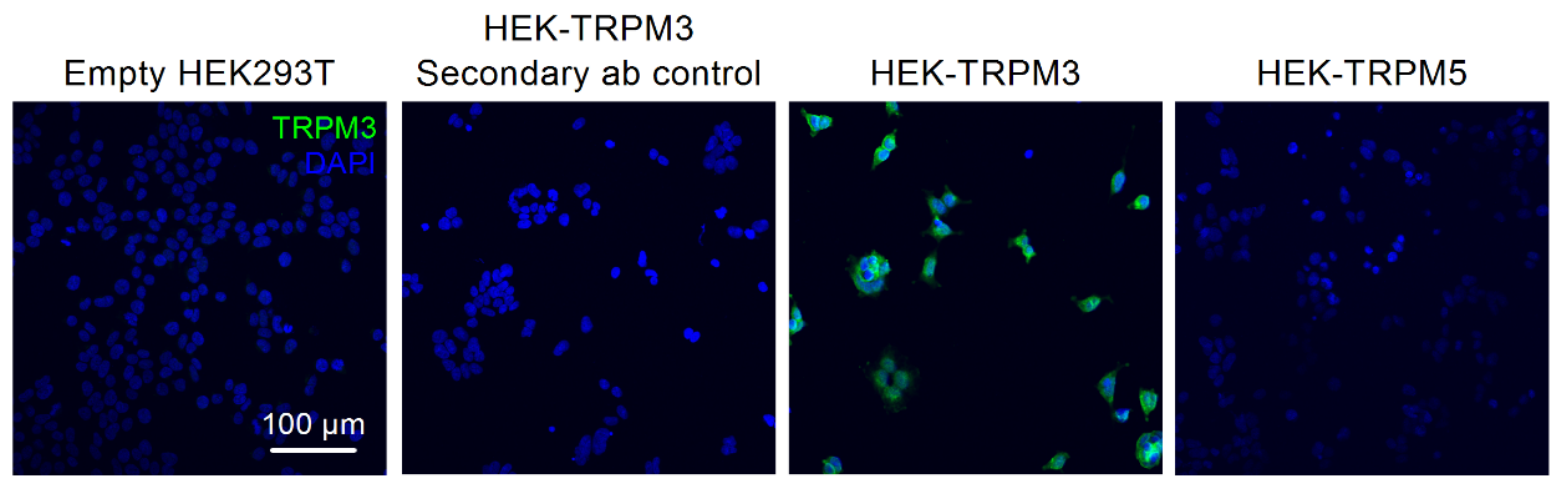

Figure 4.3. Specificity of a rabbit anti-TRPM3 antibody. Confocal images of non-transfected cells incubated with the anti-TRPM3 antibody, non-transfected cells incubated with the secondary antibody, TRPM3-transfected cells labeled with the anti-TRPM3 antibody and TRPM5-transfected cells incubated with anti-TRPM3. In all images DAPI staining is shown in blue (objective 20X). Images are representative of at least 3 independent experiments.

Of note, arteries from Trpm3 KO mice could not be used as a negative control for the TRPM3 antibody, because these animals express a truncated TRPM3 protein that can be recognized by the anti-TRPM3 antibody. However, we took advantage of the fact that these mice have incorporated a $\beta$-galactosidase ( $\beta$-gal) reporter encoded by the insertion of a $L a c-Z$ gene into the reading frame of the Trpm3 gene [142]. Using an anti- $\beta$-gal antibody in Trpm3 KO mice, we found that $\beta$-gal colocalized with the neuronal markers PGP9.5 (Figure 4.4A) and NF-200 (Figure 4.5A) and was absent in VSMC (Figure 4.5B). As expected for negative control, c57bl/6j arteries were not stained with the anti- $\beta$-gal antibody (Figure 4.4B). 


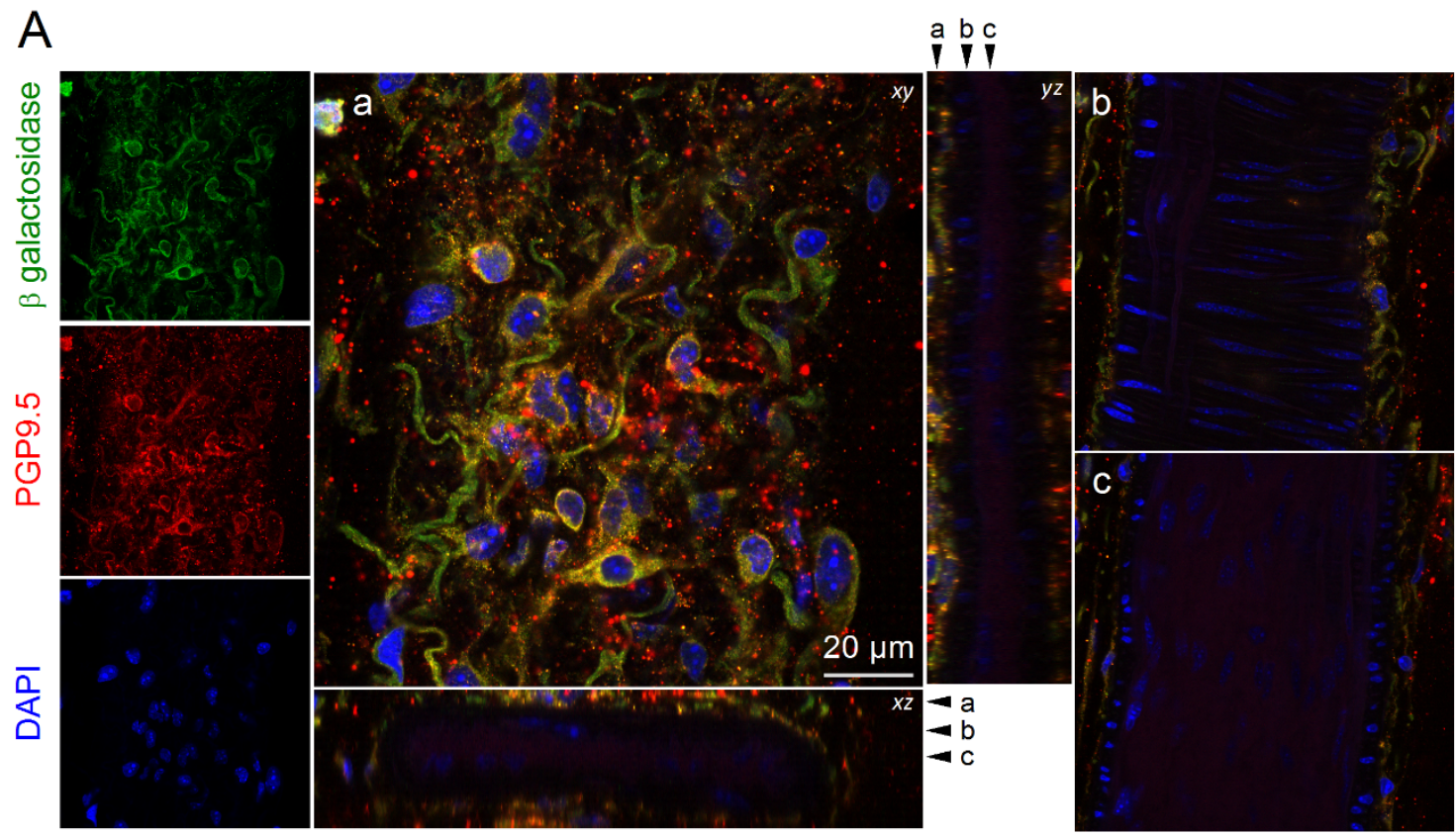

B

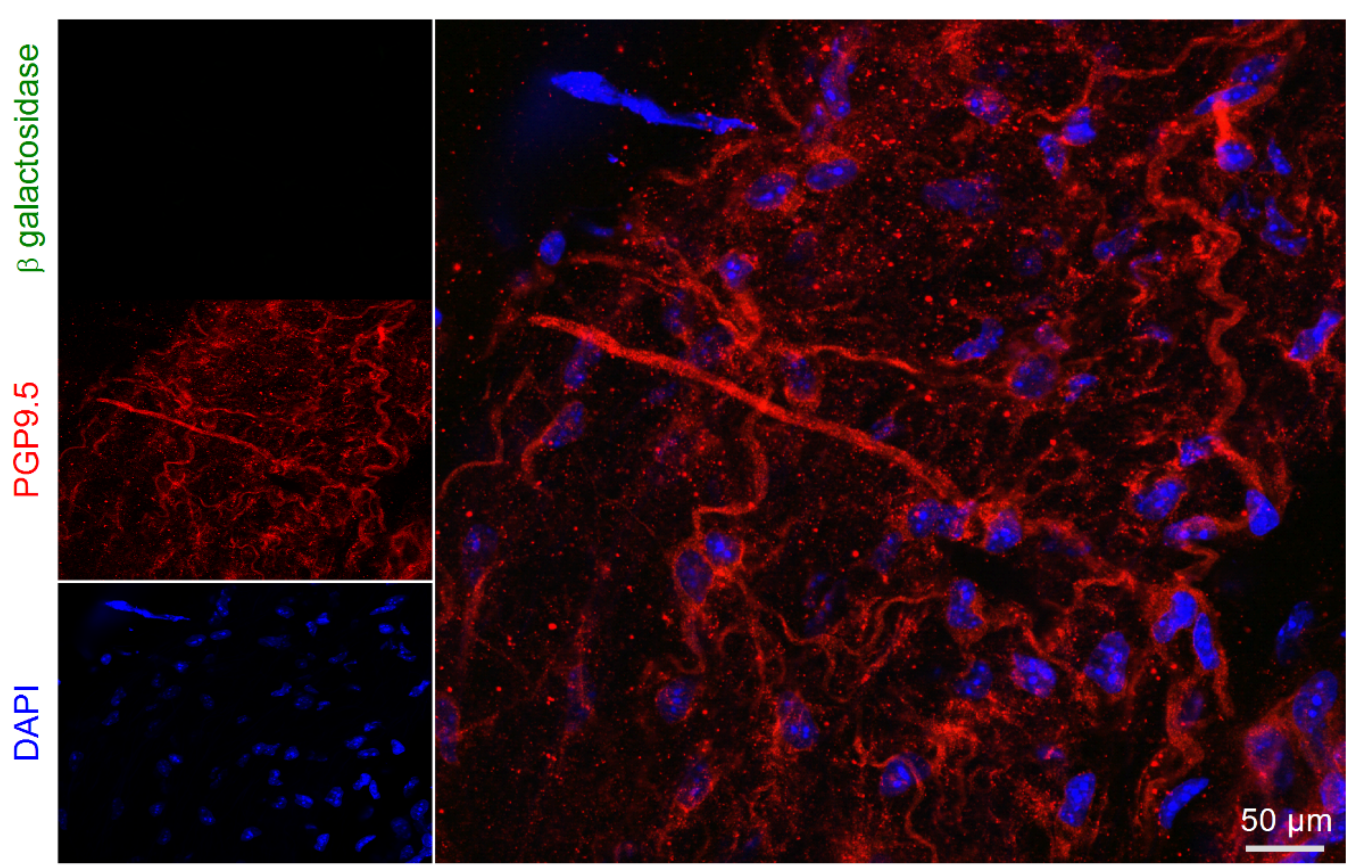

Figure 4.4. Location of the transgene product of the Trpm3 $\mathrm{KO}$ mice in perivascular nerve endings of mesenteric arteries. (A) Z-stack (31.89 $\mu \mathrm{m}$ thickness) confocal images of intact Trpm3 KO mesenteric arteries at the levels of the adventitial (a), medial (b) and endothelial (c) layers, labeled with $\beta$ galactosidase (green), PGP9.5 (red) antibodies and nuclear DAPI (blue) staining. The total images acquired were $50(z=5, b=15, c=28)$ with steps of $0.64 \mu \mathrm{m}$. The initial and final positions were set where no nuclei are visible. The images are representative of at least 3 independent experiments. $(B)$ Confocal microscopy images of the adventitial layer of a c57bl/6j mouse mesenteric artery labeled with $\beta$-galactosidase (green), PGP9.5 (red) antibodies and nuclear DAPI staining (blue). The position of the image was set according to nuclei morphology. Images are representative of 3 independent. 

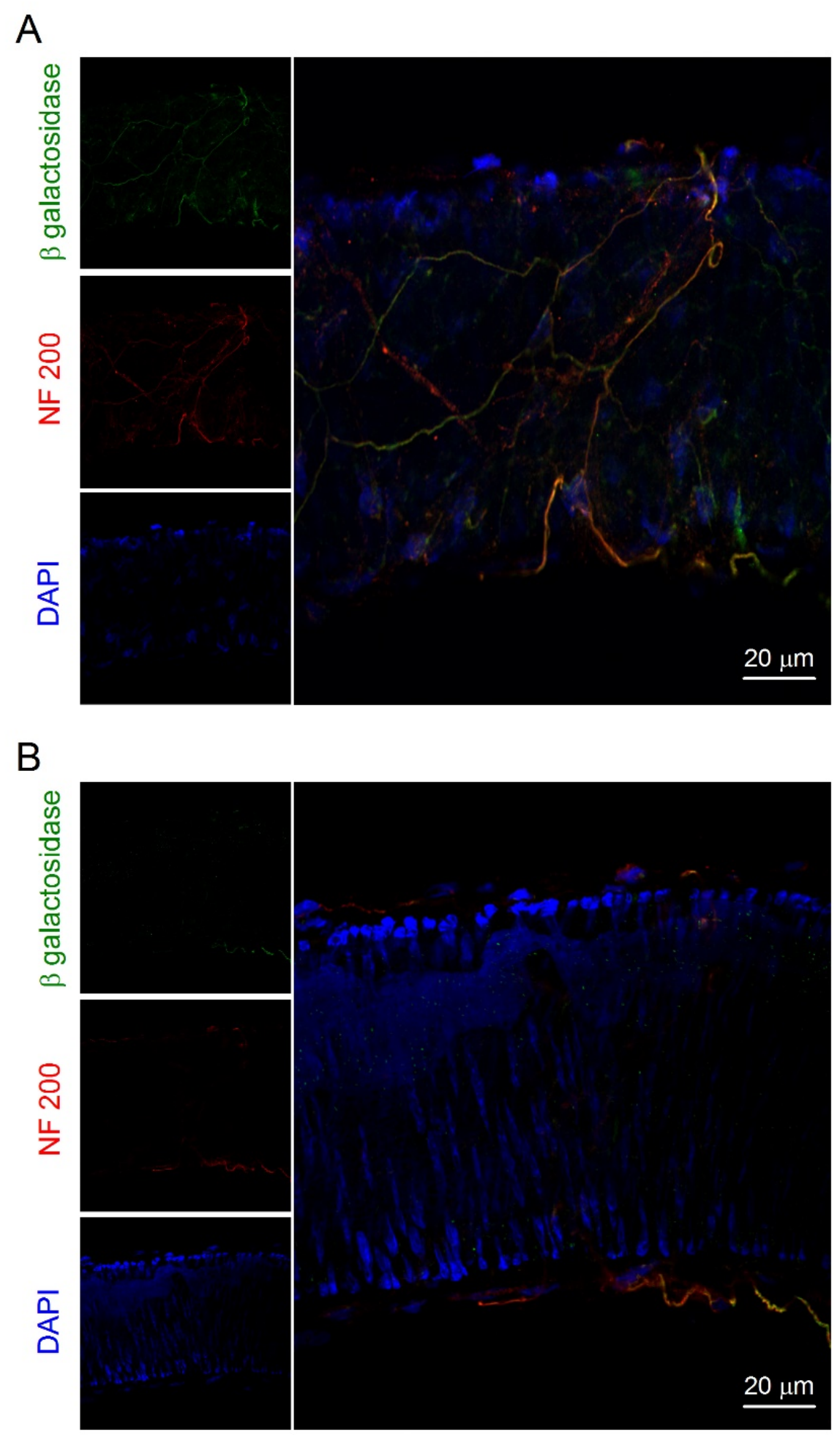

Figure 4.5. Perivascular innervation in dissected mouse mesenteric arteries. Confocal images of the adventitia $(A)$ and the medial layer $(B)$ of intact Trpm3 KO mesenteric arteries labeled with $\beta$ galactosidase (green), anti-NF-200 (red) antibodies and nuclear DAPI (blue) staining. In both images, the position was set according to nuclei morphology, 40X. Images are representative of at least 3 independent experiments. 
To further characterize the localization pattern of TRPM3 we performed double immunostaining in intact mesenteric arteries from Trpm3 KO mice labeled with antibodies against $\beta$-gal and the smooth muscle-specific protein alpha-smooth muscle actin ( $\alpha$-SMA). We found $\alpha$-SMA to be present as expected in the smooth muscle (Figure 4.6A), but not in the adventitial layer (Figure 4.6B). In contrast, $\beta$-gal was clearly detected only in nerve ending-like structures in the adventitial layer.
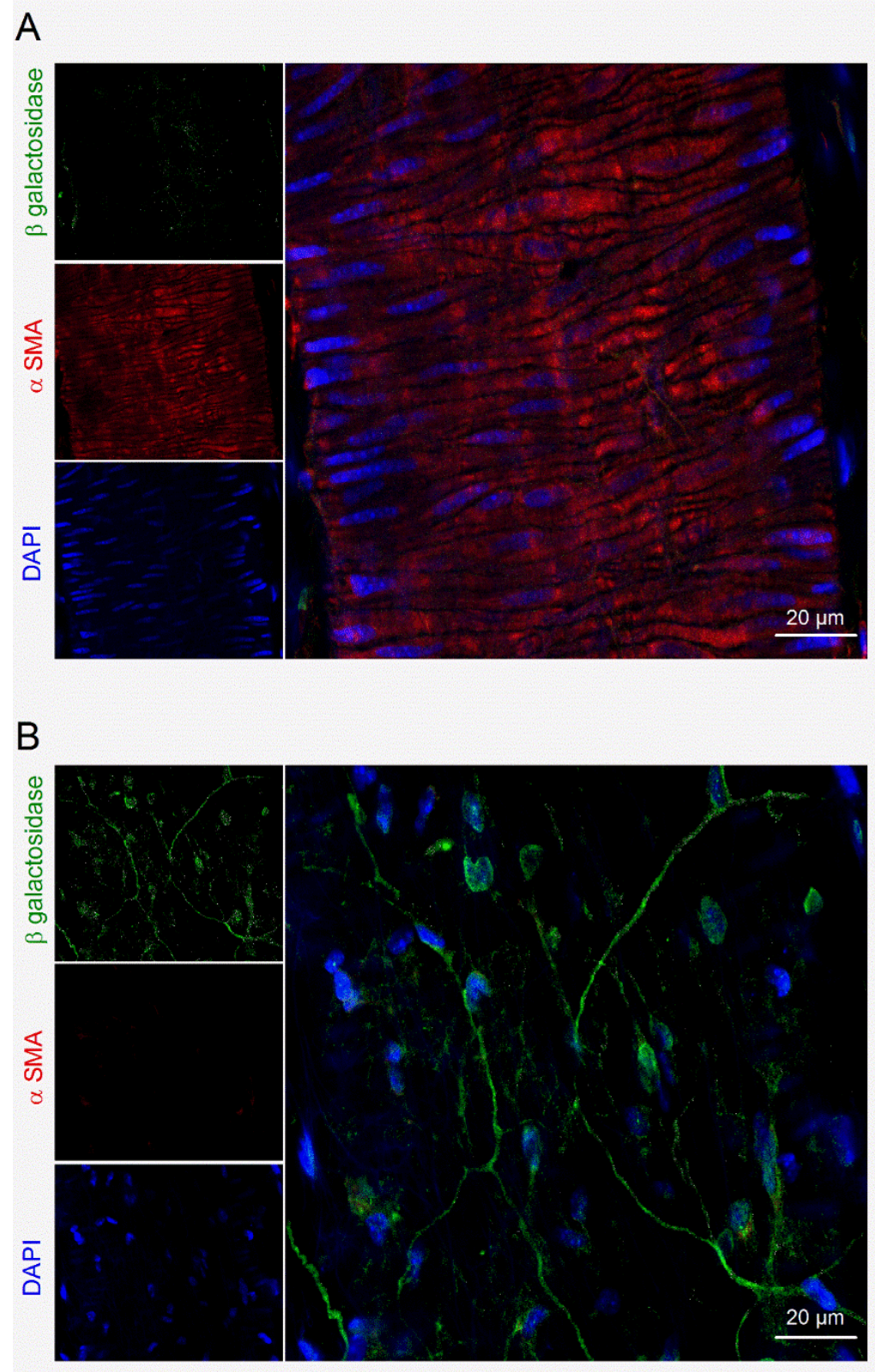

Figure 4.6. The transgene product of the Trpm3 KO mice is not located in smooth muscle layer from mesenteric arteries. Confocal microscopy images of the medial layer $(A)$ and adventital layer $(B)$ of Trpm3 KO mouse intact mesenteric arteries labeled with $\beta$-galactosidase (green), $\alpha$-smooth muscle actin (red) antibodies and nuclear DAPI staining (blue). In both images, the position was set according to nuclei morphology, 40X. Images are representative of at least 3 independent experiments. 
To test directly whether TRPM3 is functionally expressed in the medial layer of mouse mesenteric arteries we performed whole-cell patch-clamp recordings in freshly dissociated VSMC. Application of PS (10 and $30 \mu \mathrm{M})$ produced no significant change in the current amplitude at $-150 \mathrm{mV}$ and $+80 \mathrm{mV}(99.9 \pm 0.5 \%$ and $100 \pm 0.3 \%$ relative to the amplitude recorded in control condition, respectively; $\mathrm{n}=12$ cells from 4 mice; Figure $4.7 A$ ).
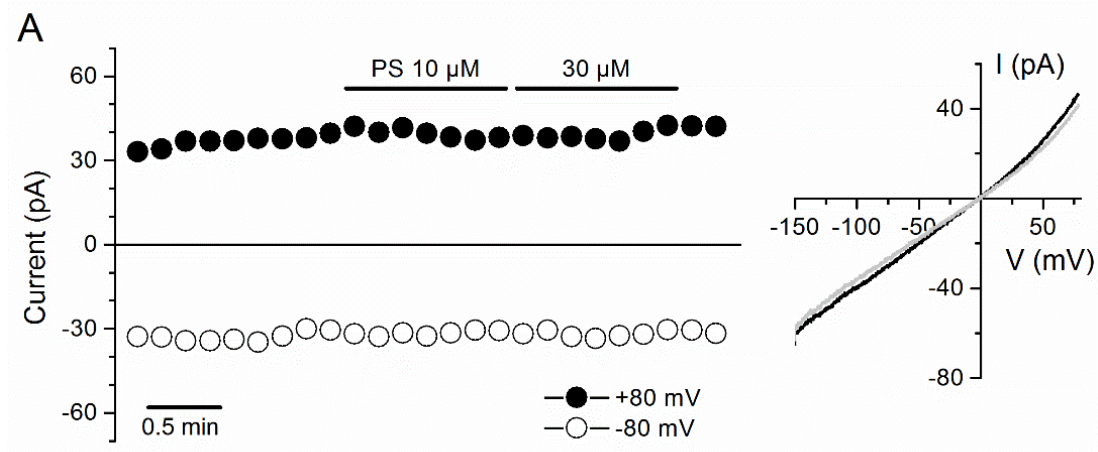

\section{B}
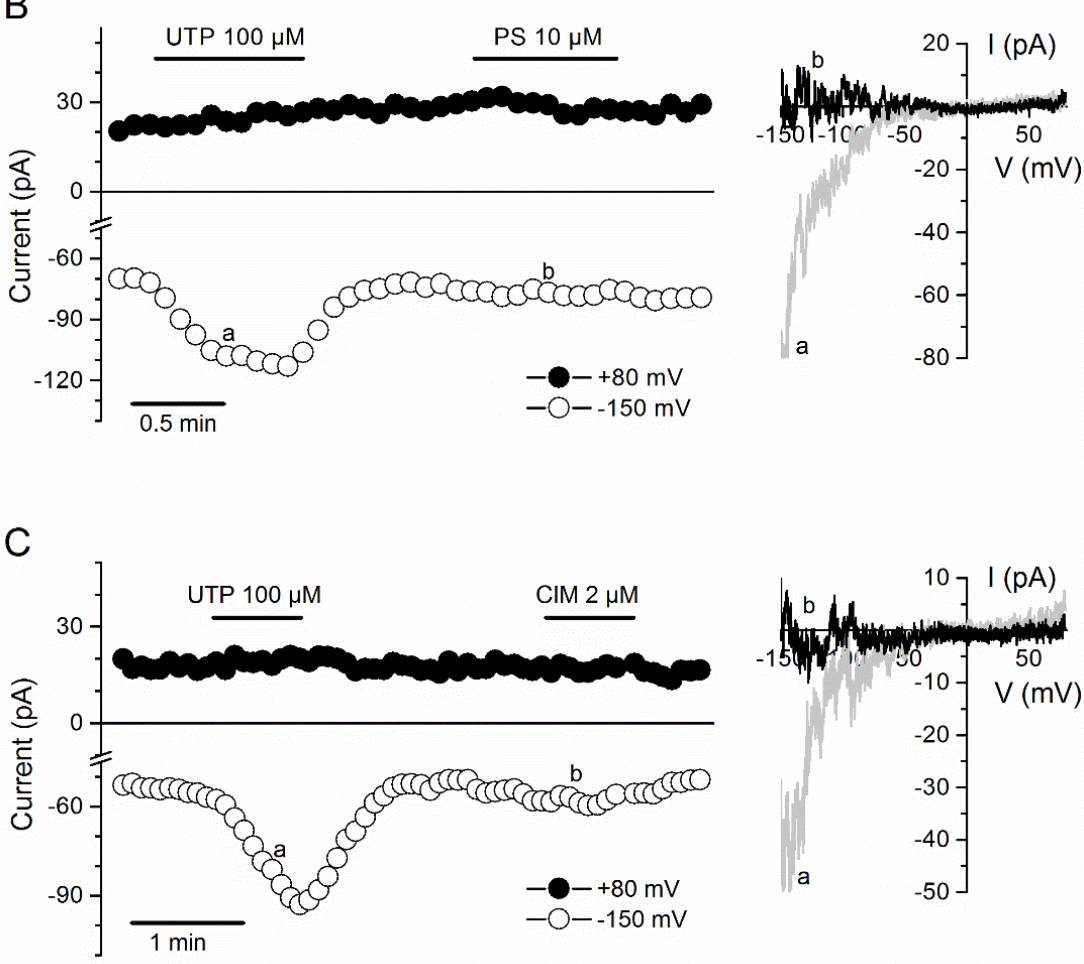

Figure 4.7. TRPM3 is not functionally expressed in c57bl/6j mouse mesenteric VSMC. (A) Left, time course of the amplitude of currents recorded at -80 and $+80 \mathrm{mV}$ in control and in the presence of 10 and $30 \mu \mathrm{M}$ PS. Right, traces recorded in control (black) and in the presence of $30 \mu \mathrm{M}$ PS (grey). $(B, C)$ Left, representative examples of the time course of the amplitude of currents recorded at -150 and $+80 \mathrm{mV}$, showing the effects of $100 \mu \mathrm{M}$ UTP, $10 \mu \mathrm{M}$ PS and $2 \mu \mathrm{M} \mathrm{CIM0216.} \mathrm{Right,} \mathrm{the} \mathrm{I-V} \mathrm{curves}$ represent the differences between traces recorded in the presence of the experimental compounds (PS, CIM and UTP) at the time points indicated by the labels $a$ and $b$ and the corresponding current trace recorded in control. 
In another series of experiments, the effects of PS $(10 \mu \mathrm{M})$ and of the potent TRPM3 synthetic agonist CIM0216 [135] on current amplitude were compared to the effects of the purinergic receptor agonist UTP [204]. Again, there was no change in current amplitude during PS application $(-0.3 \pm 0.3 \mathrm{pA}, \mathrm{P}=0.95$, at $-150 \mathrm{mV}$ and $1.3 \pm 0.9 \mathrm{pA}, \mathrm{P}=0.77$ at $+80 \mathrm{mV}(\mathrm{n}=6$ cells from 3 mice; Figure 4.7B, black trace in right panel). Currents were also unaffected by $2 \mu \mathrm{M}$ CIM0216 (current amplitude change of $-0.5 \pm 1.7 \mathrm{pA}, \mathrm{P}=0.99$ at $-150 \mathrm{mV}$ and $2 \pm 2 \mathrm{pA}, \mathrm{P}=$ 0.77 at $+80 \mathrm{mV} ; \mathrm{n}=4$ cells from 2 mice; Figure $4.7 C$, black trace in right panel). On the other hand, currents were stimulated at negative potentials by $100 \mu \mathrm{M}$ UTP, as expected. The amplitude of the UTP-sensitive current was $-51 \pm 9 \mathrm{pA}, \mathrm{P}=0.003$ at $-150 \mathrm{mV}$ and $2.7 \pm 0.7 \mathrm{pA}$, $\mathrm{P}=0.26$ at $+80 \mathrm{mV} ; \mathrm{n}=5$ cells from 2 mice (Figure $4.7 B$ and $C$, grey traces in right panels). Taken together, these data indicate the absence of functional expression of TRPM3 channels in mesenteric arteries VSMC.

\subsection{TRPM3 activation induces vasodilation mainly via stimulation of CGRP receptors}

To determine the effects of TRPM3 activation in resistance arteries we performed pressure myography experiments in endothelium-denuded mouse mesenteric arteries. Pressurized arteries were pre-contracted with $10 \mu \mathrm{M}$ phenylephrine to maintain the physiological tone and at the end of each experiment, we applied the L-type $\mathrm{Ca}^{2+}$ channel blocker nifepidine (10 $\mu \mathrm{M})$ to determine the maximum arterial diameter. Nifedipine was previously reported as a TRPM3 agonist [130]. Thus, it could be argued that nifedipine-induced vasodilation may be partly mediated by TRPM3, making this compound unsuitable for the determination of the role of this channel. To test this, we compared the vasodilation elicited in the same artery by $10 \mu \mathrm{M}$ nifedipine application and by perfusion with $\mathrm{Ca}^{2+}$-free bath solution. We observed similar increase in the arterial diameter in both cases (Figure 4.8). This demonstrates that 10 $\mu \mathrm{M}$ of nifedipine has the same effect than abrogating $\mathrm{Ca}^{2+}$ entry through L-type $\mathrm{Ca}^{2+}$ channels, thus validating its use to determine the maximal vasodilation.

PS induced a dose-dependent reversible vasodilation in arteries dissected from c57bl/6j animals. The data was best fit by the sum of two Hill functions, suggesting for at least two targets of PS (Figure 4.9A and D). The $E C_{50}$ values for these components were $14 \pm 2 \mu \mathrm{M}$ and $100 \pm 9 \mu \mathrm{M}$ and the corresponding Hill coefficients $(H)$ were $2.2 \pm 0.5$ and $4.3 \pm 1.2$, respectively. This fitting also yielded a value of relative amplitude of the low $E C_{50}$ vasodilation 
component of $57 \pm 7 \%(A 1$, see the two-Hill components equation in the Methods, section Pressure myography experiments). To determine the contribution of TRPM3 channels to the effects of PS we measured the response of mesenteric arteries isolated from Trpm3 KO mice. PS dilated Trpm3 KO arteries only at concentrations higher than $\sim 10 \mu \mathrm{M}$ (Figure 4.9B and D).

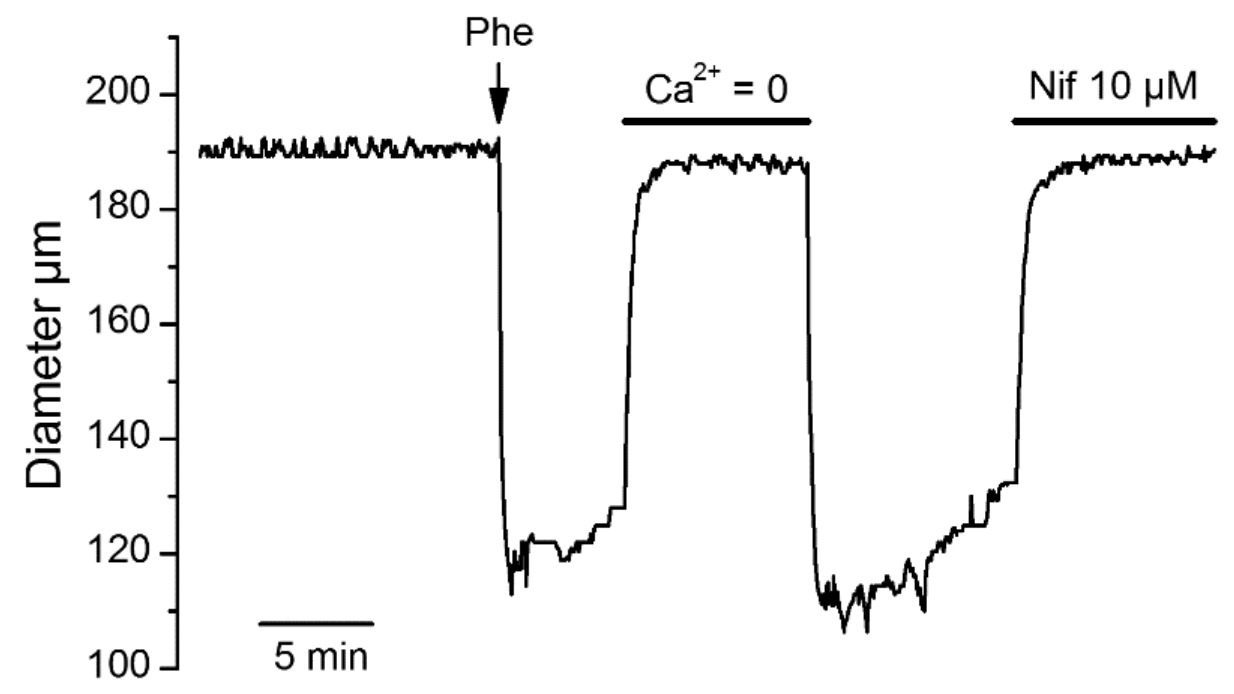

Figure 4.8. Nifedipine $(10 \mu \mathrm{M})$ induces maximal vasodilation in mouse mesenteric arteries. Comparison of the vasodilating effects of a $\mathrm{Ca}^{2+}$-free solution and $10 \mu \mathrm{M}$ nifedipine (Nif) in c57bl/6j arteries. Phenylephrine is defined as Phe.

The dose-response curve for Trpm3 KO arteries could be fitted with a single Hill function, with $E C_{50}$ and Hill values of $53 \pm 3 \mu \mathrm{M}$ and $2.2 \pm 0.2$, respectively. These results further support the idea that in c57bl/6j arteries there are two different mechanisms involved in PS-induced vasodilation, being only the one with lower $E C_{50}$ TRPM3-dependent.

In addition, CIM0216 induced a dose-dependent reversible vasodilation in arteries from c57bl/6j animals, with $E C_{50}$ and Hill coefficients values of $0.40 \pm 0.05 \mu \mathrm{M}$ and $0.65 \pm 0.06$, respectively (Figure 4.10A and C). Arteries dissected from Trpm3 $\mathrm{KO}$ mice responded to this compound, but at concentrations higher than $0.1 \mu \mathrm{M}$, with $E C_{50}$ and Hill coefficient values of $1.50 \pm 0.08 \mu \mathrm{M}$ and $1.6 \pm 0.1$, respectively (Figure $4.10 B$ and $C$ ). 

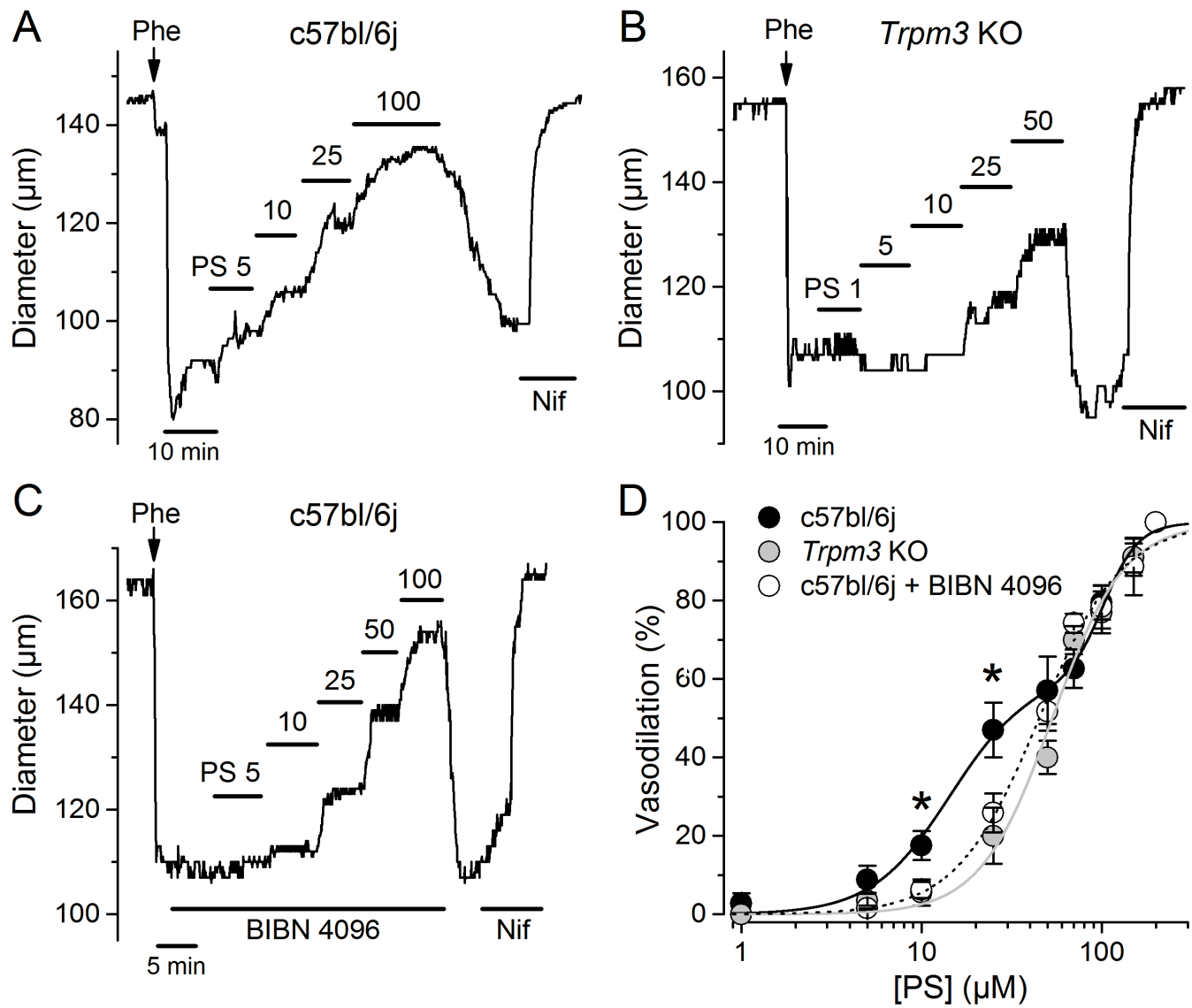

E

$\beta$ galactosidase

CGRP

$\beta$ galactosidase + CGRP
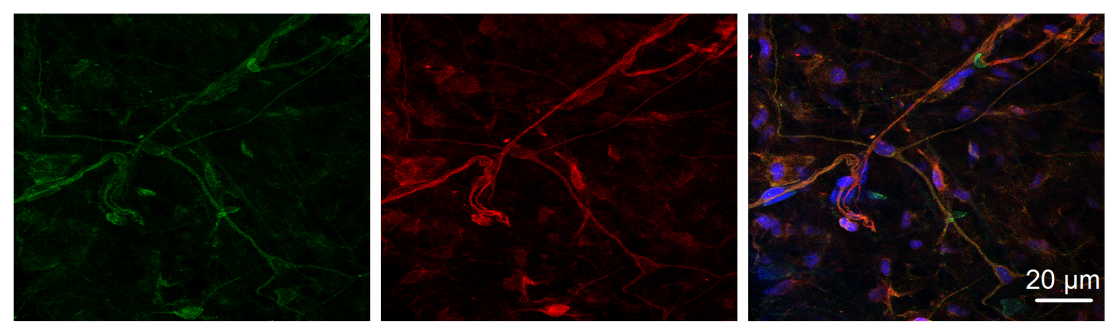

Figure 4.9. TRPM3-induced vasodilation of mesenteric arteries is mediated by CGRP receptor activation. Representative examples of the effects of increasing concentrations of pregnenolone sulfate (PS, in $\mu \mathrm{M}$ ) on the diameter of arteries dissected from c57bl/6j $(A)$ and Trpm3 KO mice $(B)$ in the presence of phenylephrine (Phe, $10 \mu \mathrm{M}$ ). (C) Effects of PS (in $\mu \mathrm{M}$ ) on c57bl/6j arteries in the presence of the CGRP receptor antagonist BIBN $4096(1 \mu \mathrm{M})$ and phenylephrine $(10 \mu \mathrm{M})$. Nifedipine (Nif, $10 \mu \mathrm{M}$ ) was applied at the end of each experiment. (D) Dose dependency of PS-induced vasodilation (in $\mu \mathrm{M}$ ) in precontracted arteries dissected from c57bl/6j, Trpm3 $\mathrm{KO}$ mice and in c57bl/6j arteries in the presence of $1 \mu \mathrm{M}$ BIBN 4096. Data are mean \pm SEM (c57/bl6j, $\mathrm{n}=10 ; \operatorname{Trpm} 3 \mathrm{KO}, \mathrm{n}=8$ and $c 57 \mathrm{bl} / 6 \mathrm{j}+\mathrm{BIBN} 4096, \mathrm{n}=8$ ). The black solid line represents the fit of the $\mathrm{c} 57 \mathrm{bl} / 6 \mathrm{j}$ data with a twocomponent Hill equation. The grey solid line and the dotted line represent the fit of the data for Trpm3 $\mathrm{KO}$ and $\mathrm{c57bl} / 6 \mathrm{j}$ in the presence of BIBN 4096 with single-component Hill equations. ${ }^{*}$ indicates $P<$ 0.05 compared to c57bl/6j mice, unpaired t-test. (E) Confocal images of intact Trpm3 KO mouse mesenteric arteries of the adventitial layer labeled with $\beta$-galactosidase (green), CGRP (red) antibodies and nuclear DAPI staining (blue). The position was set according to the nuclei morphology, 40X. Images are representative of at least 3 independent experiments. 

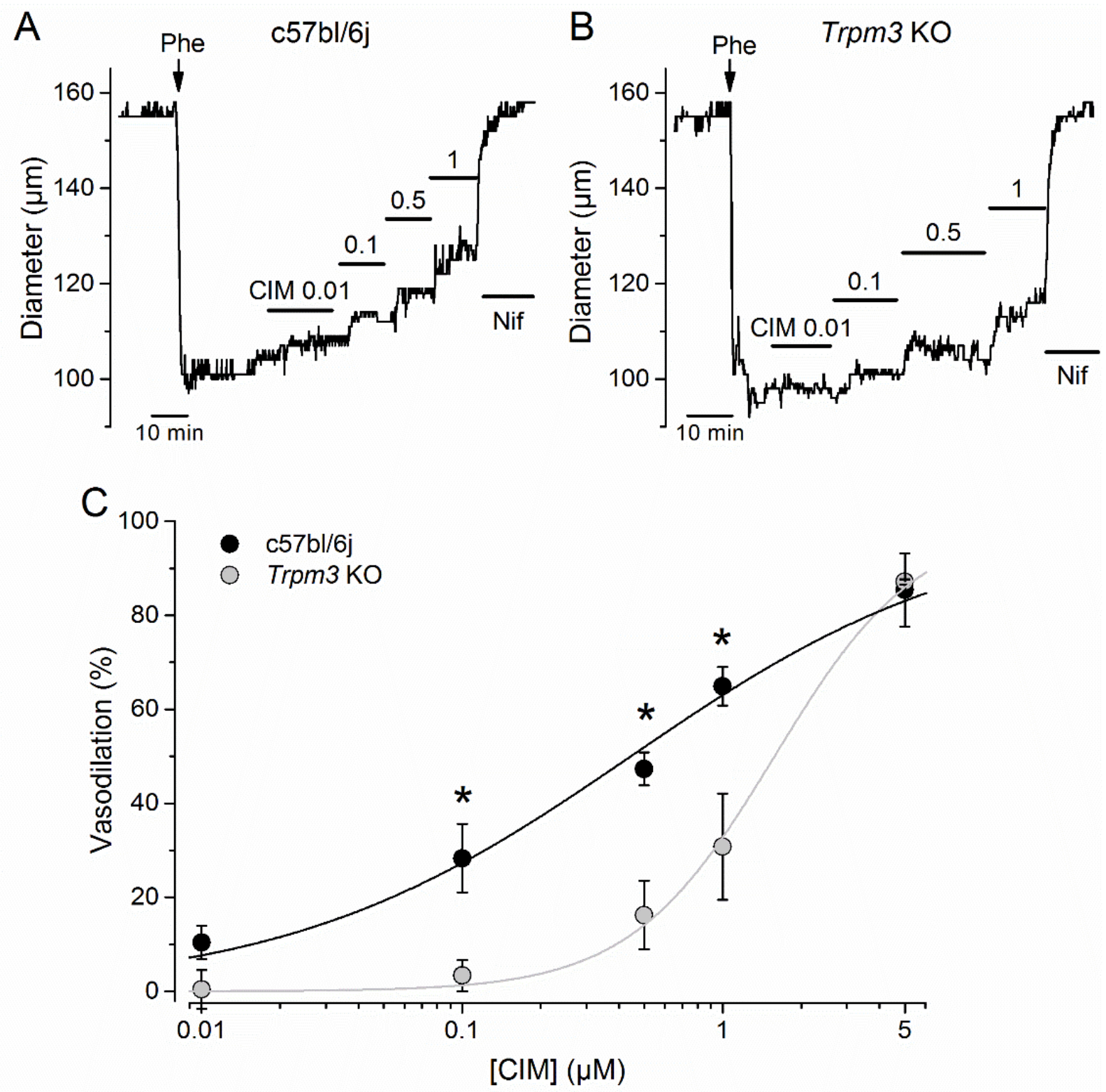

Figure 4.10. A synthetic potent agonist of TRPM3 induces vasodilation of endothelium-denuded mesenteric arteries. Representative examples of the effects of $\mathrm{CIM0216}$ at different concentrations applied on the diameter of arteries dissected from c57bl/6j $(A)$ and Trpm3 KO mice $(B)$. (C). Dose dependency of CIM0216-induced vasodilation in arteries dissected from c57bl/6j and Trpm3 KO mice. Nifedipine (Nif, $10 \mu \mathrm{M}$ ) was applied at the end of each experiment. Data are mean \pm SEM (c57/bl6j, $n$ $=4$ and Trpm3 KO, $\mathrm{n}=4$ ). The black and grey solid lines represent the fit of the $\mathrm{c57} / \mathrm{bl} / 6 \mathrm{j}$ and Trpm $3 \mathrm{KO}$ data, respectively, with a single-component Hill equation. Phe $=$ Phenylephrine. ${ }^{*}$ indicates $P<0.05$ compared to $\mathrm{c} 57 \mathrm{bl} / 6 \mathrm{j}$ mice, paired t-test.

Based on prior studies on sensory TRP channels such as TRPV1 and TRPA1 $[102,205]$ and the expression of TRPM3 in nociceptive neurons [62, 135], as well as the well-stablished neuroanatomical localization of CGRP in perivascular nerves innervating the adventitial layer [206-209], we hypothesized that the TRPM3-dependent vasodilation induced by PS may be 
mediated by the release of CGRP and the relaxing effect of this peptide on VSMC. To test this, we performed myography experiments in the presence of the CGRP receptor antagonist, BIBN 4096. In this condition, PS-induced vasodilation could only be elicited at concentrations above 10-15 $\mu \mathrm{M}$ (Figure 4.9C). The dose-response curve of the effect of PS in the presence of BIBN $4096(1 \mu \mathrm{M})$ was similar to the curve obtained in arteries from Trpm3 KO mice, with $E C_{50}$ and $H$ values of $44 \pm 2 \mu \mathrm{M}$ and $1.8 \pm 0.1$, respectively (Figure 4.9D). Furthermore, double immunolabelling of intact mesenteric arteries from Trpm3 KO mice with anti- $\beta$-gal and antiCGRP antibodies showed a good colocalization in the sensory fibres that innervate the adventitial layer (Figure 4.9E). These results indicate that TRPM3 location seems to be restricted to sensory nerve endings, where the TRPM3-mediated effect of PS depends on CGRP receptor activation.

The vasodilating action of CGRP has endothelium-dependent and endothelium independent components, which are mediated by the stimulation of CGRP receptors in endothelial cells and VSMC, respectively $[19,22]$. Thus, the TRPM3-dependent effect of PS is expected to be stronger in the presence of endothelium. We found that this was indeed the case, as PS induced a dose-dependent biphasic effect in intact arteries (Figure 4.11A), with $E C_{50}$ values lower than the corresponding ones found in endothelium denuded preparations $(8.1 \pm 0.5 \mu \mathrm{M}$ and $50 \pm 2 \mu \mathrm{M})$ (Figure 4.11B).

\subsection{CGRP release induces vasodilation via activation of $\mathrm{K}^{+}$channels}

Next, we explored the mechanisms by which CGRP receptor activation in VSMC leads to vasodilation. Stimulation of the CGRP receptor has been reported to increase cyclic adenosine monophosphate (cAMP) production via the adenylate cyclase (AC) [20] and may therefore lead to protein kinase $\mathrm{A}$ (PKA)-mediated activation of $\mathrm{K}^{+}$channels in VSMC hyperpolarization and arterial relaxation.

To test whether activation of $\mathrm{K}^{+}$channels is involved in the vasodilation response triggered by TRPM3 activation we compared the effects of $10 \mu \mathrm{M}$ PS in arteries treated or not with $\mathrm{K}^{+}$ channel blockers. We noticed that the vasodilation induced by acute application of $10 \mu \mathrm{M}$ PS $(42 \pm 4 \%)$ in the presence of phenylephrine $(10 \mu \mathrm{M})$ (Figure 4.13A) was larger than that induced by the same concentration during the cumulative dose-response experiments (18 \pm $4 \%$ ). This may be due to partial CGRP depletion induced by previous applications of PS at low 
concentrations. Nevertheless, the effects of cumulative (Figure 4.9D) or acute (Figure 4.12) application of $10 \mu \mathrm{M}$ PS were largely mediated by CGRP.

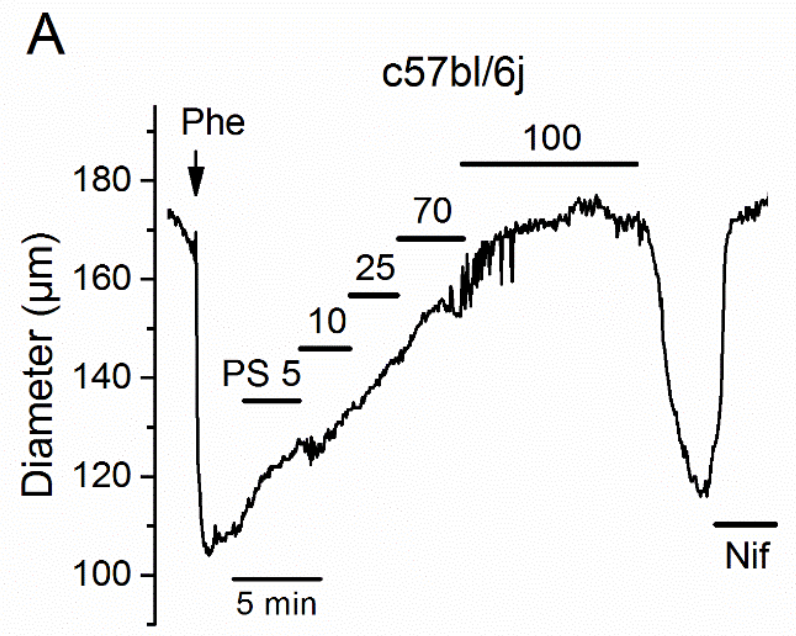

B

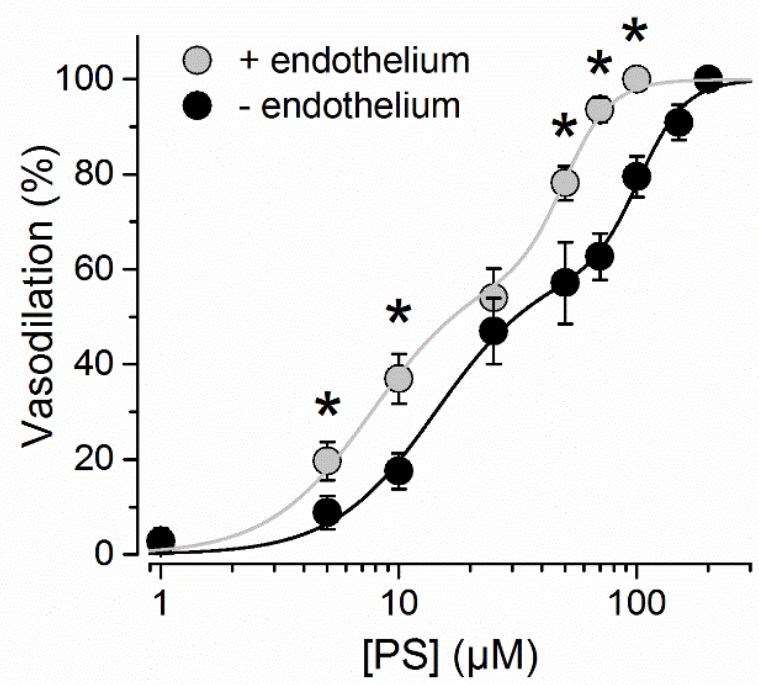

Figure 4.11. TRPM3-induced vasodilation of mesenteric arteries is enhanced by endothelium. (A) Representative example of the vasodilation induced by PS in a c57bl/6j mouse mesenteric artery with endothelium precontrated with phenylephrine $(10 \mu \mathrm{M})$. At the end of each experiment, Nif $(10 \mu \mathrm{M})$ was applied. (B) Comparison of the dose responses to PS in precontrated arteries with endothelium ( $\mathrm{n}$ $=8$ ) and without endothelium ( $n=10$, same data as in Figure 5.8D is shown for comparison). The solid lines represent the best fit of the data with two-component Hill equations. ${ }^{*}$ indicates $P<0.05$ compared to the corresponding data for $\mathrm{c} 57 \mathrm{bl} / 6 \mathrm{j}$ without endothelium, unpaired t-test.

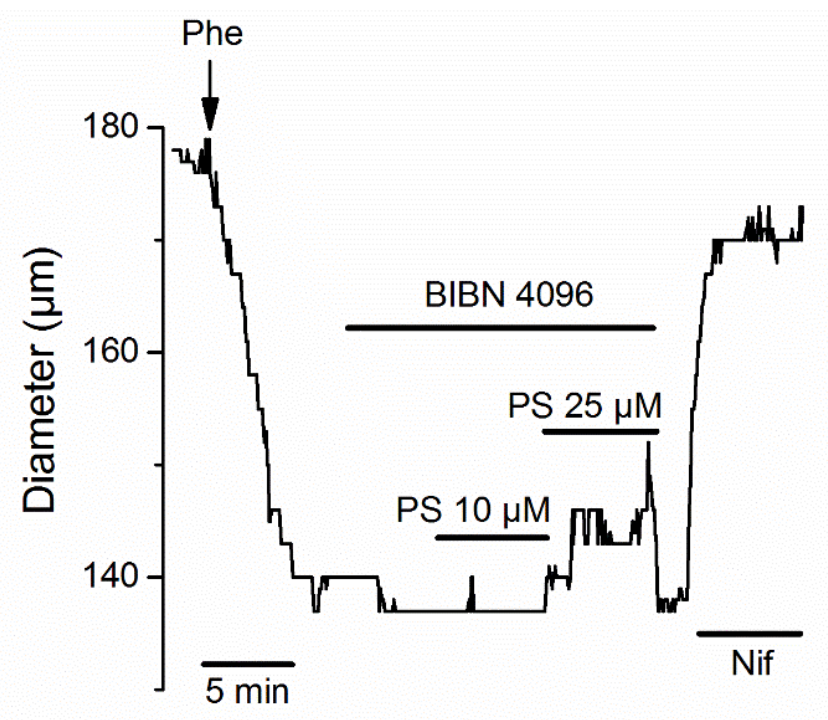

Figure 4.12. PS-induced vasodilation is markedly reduced in the presence of the CGRP antagonist. Effects of PS $(10 \mu \mathrm{M}$ and 25 $\mu \mathrm{M})$ on a $c 57 \mathrm{bl} / 6 \mathrm{j}$ mouse mesenteric artery in the presence of the CGRP receptor antagonist BIBN 4096. Phe = Phenylephrine, $\mathrm{PS}=$ pregnenolone sulfate and $\mathrm{Nif}=$ nifedipine.

Pretreatment with the voltage- and $\mathrm{Ca}^{2+}$-activated $\mathrm{K}^{+}$channel blocker paxilline (500 nM) [210] and the $\mathrm{K}_{\mathrm{V} 1}$ channels blocker correolide $(10 \mu \mathrm{M})$ [211] led to a significant reduction of the 
effect of PS on pre-contracted mesenteric arteries. This effect was enhanced by addition of the $\mathrm{K}_{\mathrm{V} 2}$ blocker stromatoxin (ScTx1, $50 \mathrm{nM}$ ) (Figure 4.13A and B) [212].
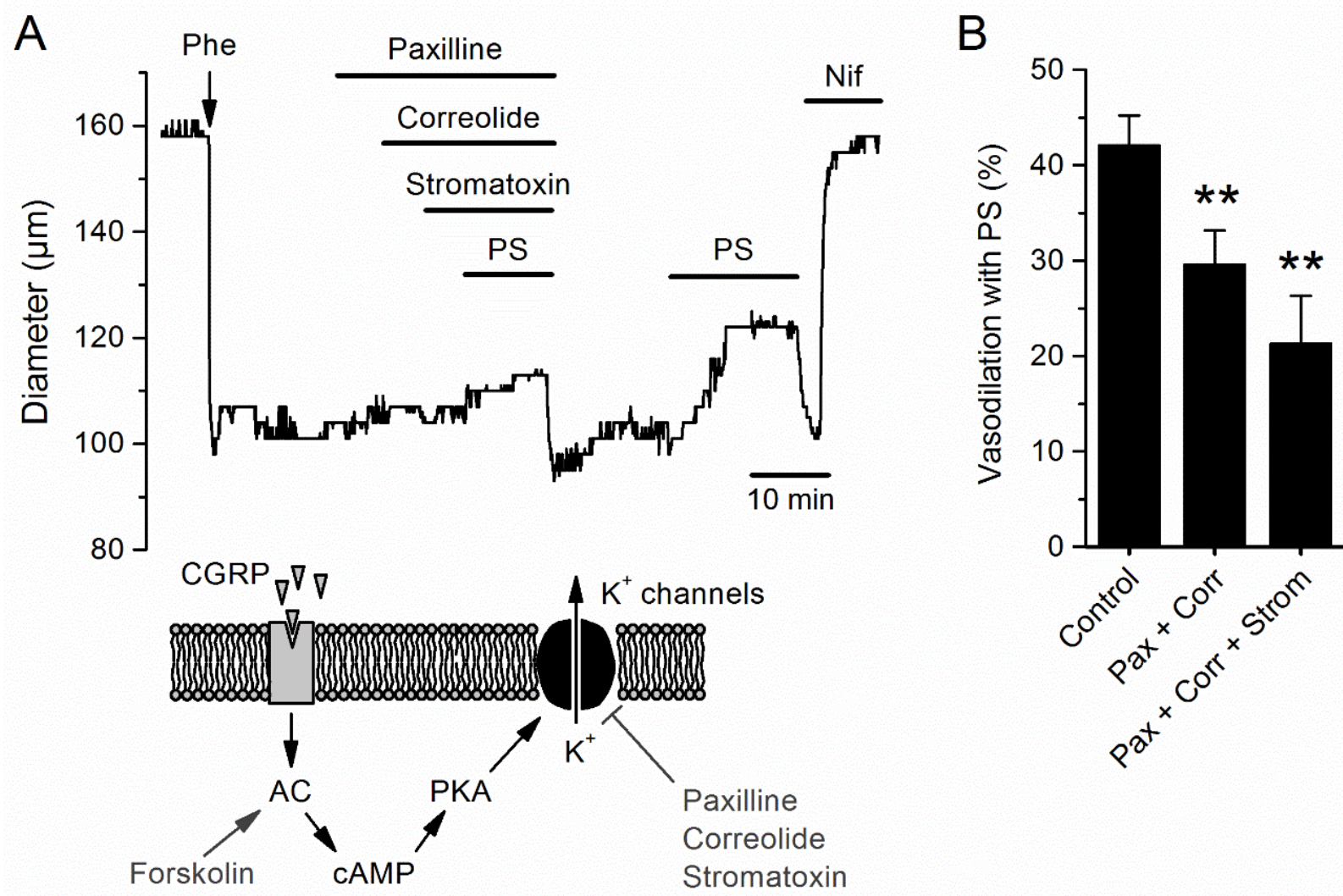

Figure 4.13. TRPM3-dependent vasodilation is partly mediated by activation of $\mathrm{K}^{+}$channels. $(A)$ Comparison of the effects of pregnenolone sulfate (PS, $10 \mu \mathrm{M}$ ) in control conditions and in the presence of the $\mathrm{K}^{+}$channel blockers paxilline $(500 \mathrm{nM})$, correolide $(10 \mu \mathrm{M})$ and stromatoxin $(50 \mathrm{nM})$. The scheme shows the CGRP signaling cascade leading to activation of $\mathrm{K}^{+}$channels via stimulation of adenylate cyclase (AC) and protein kinase A (PKA) in VSMC. (B) Average vasodilator effects of $10 \mu \mathrm{M}$ PS on $c 57 \mathrm{bl} / 6 \mathrm{j}$ mesenteric arteries in control conditions $(n=14)$, in the presence of paxilline and correolide $(n=8)$ and in the presence of paxilline, correolide and stromatoxin $(n=5) . * *$ indicate $P<$ 0.05 for the comparison with the data obtained in control, unpaired t-test. Phe = Phenylephrine; Nif = nifedipine.

None of these compounds affected the responses of HEK293T cells stably transfected with mouse TRPM3 to PS (Figure 4.14A), indicating that they did not target TRPM3 in the arterial preparations. We also probed for direct modulatory effects of PS on $\mathrm{K}_{v}$ channels in whole-cell patch-clamp experiments performed in mesenteric VSMC freshly isolated from c57bl/6j and Trpm3 KO mice. Current-voltage relationships elicited in c57bl/6j and in Trpm3 KO VSMC by depolarizing pulses were unaffected by application of PS (10 and $30 \mu \mathrm{M}$; Figure 4.14B and C). 

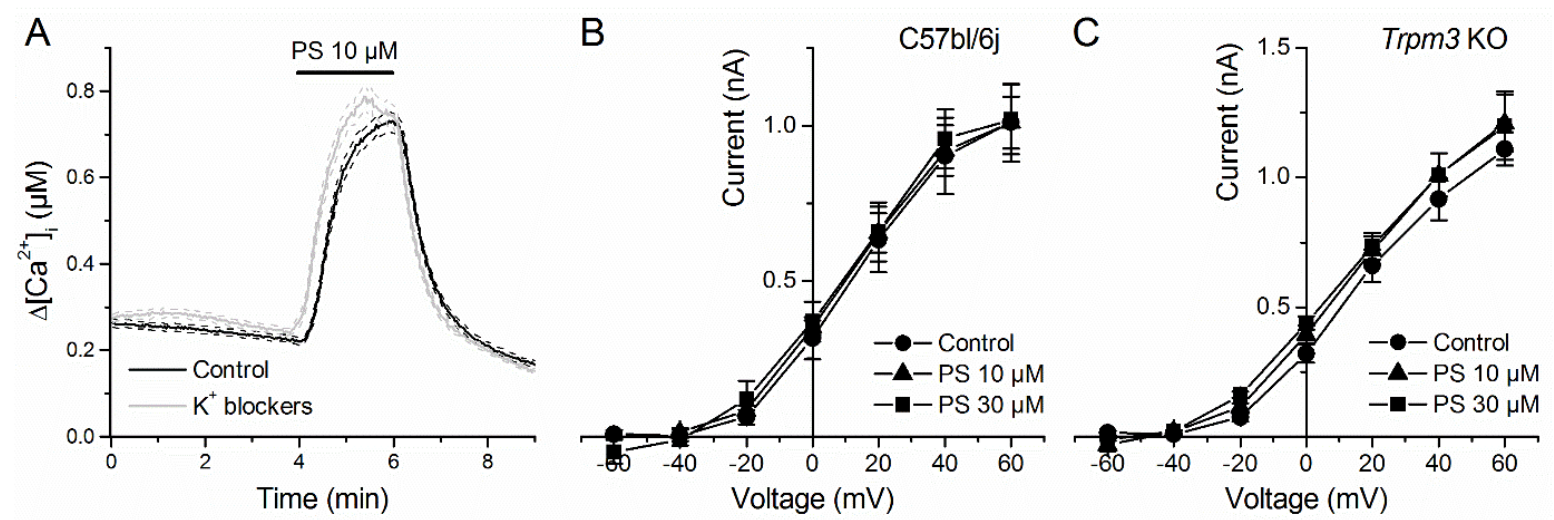

Figure 4.14. Absence of non-specific effects of $\mathrm{K}^{+}$channel blockers on TRPM 3 channels or PS on $\mathrm{K}^{+}$ channels. $(A)$ Intracellular $\mathrm{Ca}^{2+}$ signals in HEK293T-TRPM3 cells stimulated with $10 \mu \mathrm{M}$ PS in control ( $\mathrm{n}$ = 25) was not affected by the presence of a cocktail of $\mathrm{K}^{+}$channel blockers including paxilline, correolide and stromatoxin $(n=25)$. Voltage-dependence of the amplitude of $K^{+}$currents recorded VSMC isolated from c57bl/6j $(n=10)(B)$ and $\operatorname{Trpm} 3 \mathrm{KO}(\mathrm{n}=10)(C)$ mice in control and in the presence of 10 or $30 \mu \mathrm{M}$ PS.

We further assessed the implication of the CAMP pathway and $\mathrm{K}^{+}$channels by testing the effects of forskolin, a direct activator of AC, both in the absence and in the presence of paxilline, correolide and stromatoxin. We found $1 \mu \mathrm{M}$ forskolin induce strong vasodilation, an effect that was significantly attenuated in the presence of the $\mathrm{K}^{+}$channel blockers (Figure 4.15A and B). Taken together, these results indicate that the TRPM3-mediated dilation of mesenteric arteries is at least partly mediated by the activation of $\mathrm{K}_{\mathrm{V}}$ channels in VSMC.

\subsection{Sympathetic nerves are not implicated in TRPM3-mediated vasodilation}

The tone of mesenteric arteries is regulated by sympathetic innervation through the release of noradrenaline. The dominant effect of noradrenaline on mesenteric arteries is $\alpha_{1}$ adrenoreceptor-mediated vasoconstriction, but mesenteric VSMC also express $\beta_{2^{-}}$ adrenoreceptors, whose activation could induce vasodilation. If TRPM3 channels were also expressed in sympathetic nerve endings, PS-induced dilation of mesenteric arteries could be partly mediated by the activation of $\beta_{2}$-adrenoreceptors. We used several approaches to assess this possibility. First, we studied the functional contribution of $\beta_{2}$-adrenoreceptors to the sympathetic response. The application of $20 \mu \mathrm{M}$ noradrenaline led to a vasoconstriction that was not affected by the application of the selective $\beta_{2}$ - antagonist propranolol ( $1 \mu \mathrm{M}$ and $5 \mu \mathrm{M}$, Figure 4.16A). This suggests that the effects of sympathetic stimulation on mesenteric vessels are exclusively mediated by noradrenaline acting on $\alpha_{1}$-adrenoreceptors. Consistently with this, we failed to find any vasodilation in response to noradrenaline application in Phe- 
pre-contracted arteries (Figure 4.16B). In another series of experiments, we found that PS (10 $\mu \mathrm{M}$ ) induced vasodilation in the presence of $5 \mu \mathrm{M}$ propranolol (Figure 4.16C, $40.3 \pm 1.2 \mu \mathrm{M}, \mathrm{n}$ $=3$ ), further indicating that $\beta_{2}$-adrenoreceptors are not involved in the vasodilation induced by PS.
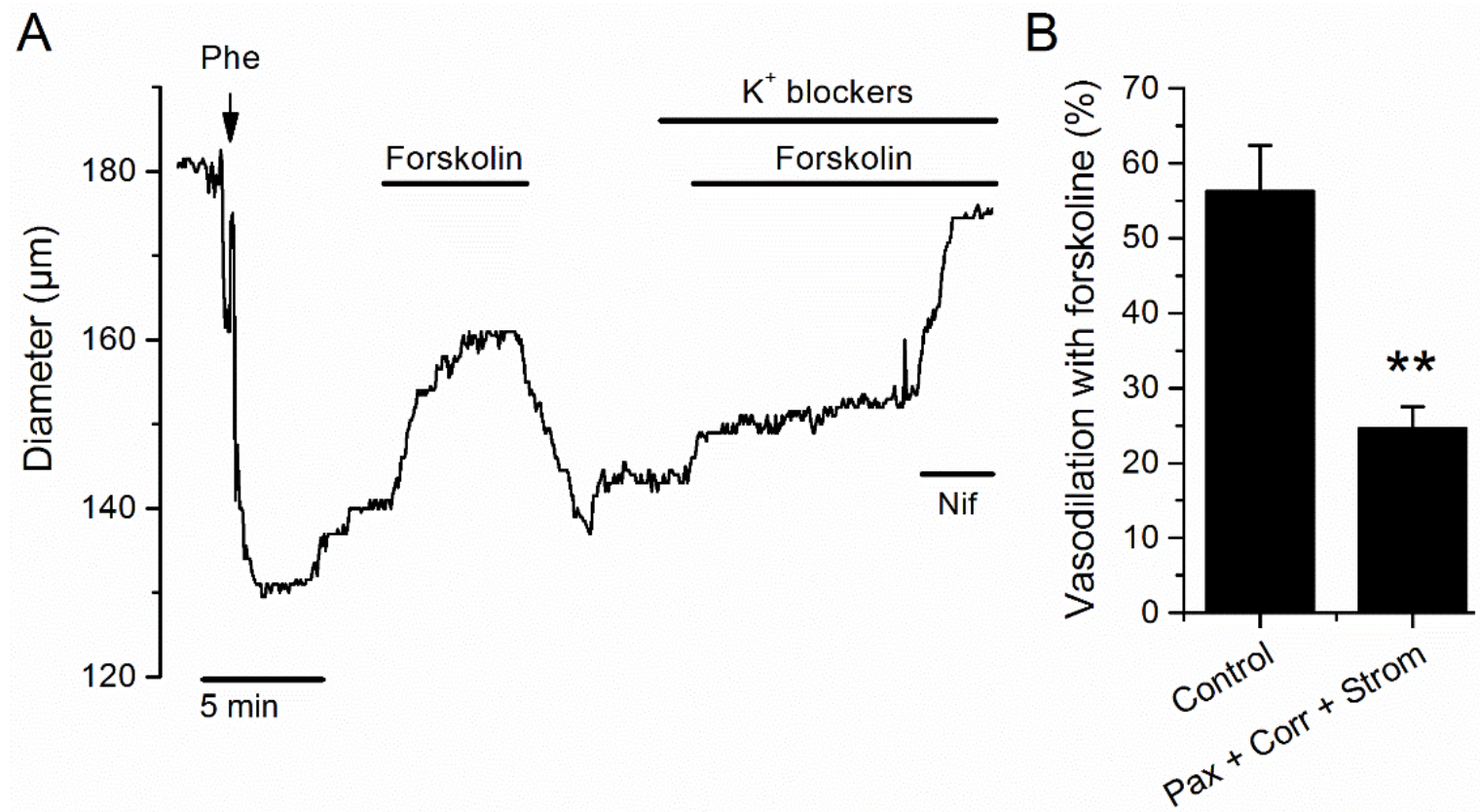

Figure 4.15. Stimulation of adenylate cyclase induces a strong vasodilation attenuated in the presence of $\mathrm{K}^{+}$channels. (A) Representative example of the effect of $1 \mu \mathrm{M}$ forskolin on a c57bl/6j mouse mesentery artery in control and in the presence of the $\mathrm{K}^{+}$channel blockers paxilline $(500 \mathrm{nM})$, correolide $(10 \mu \mathrm{M})$ and stromatoxin $(50 \mathrm{nM})$. (B) Average vasodilator effect of $1 \mu \mathrm{M}$ forskolin in the absence $(n=4)$ and in the presence of $K^{+}$channel blockers $(n=4)$. Phe $=$ Phenylephrine; Nif $=$ nifedipine. ** indicates $P<0.05$ versus control, paired t-test.

In addition, we performed double immunostainings of intact mesenteric arteries of Trpm3 KO mice using anti- $\beta$-gal and anti-Tyrosine hydroxylase (TH) antibodies. We found $\beta$-gal positive structures in the adventitia that were clearly not stained for TH (Figure 4.17), white arrowheads in the bottom-left panel). We did observe some overlap between $\beta$-gal- and THpositive structures, but it seems that this was due to their close proximity and not to an actual co-expression of $\beta$-gal and TH in the same nerve endings (Figure 4.16, adventitia, left panels). The reason for this is that the $\beta$-gal labeling, but not the TH one, was progressively lost, as the images were taken closer to the medial layer (Figure 4.17, advent. + VSMC, right panels). Note that it seems highly unlikely that $\beta$-gal and TH would localize in the same fibres and that only the expression of the former, being an exogenous and probably unregulated protein, suddenly stops at the points of fibres entry into the medial layer. Altogether, our functional and 
anatomical experiments point to a lack of involvement of sympathetic fibres in TRPM3mediated vasodilation.
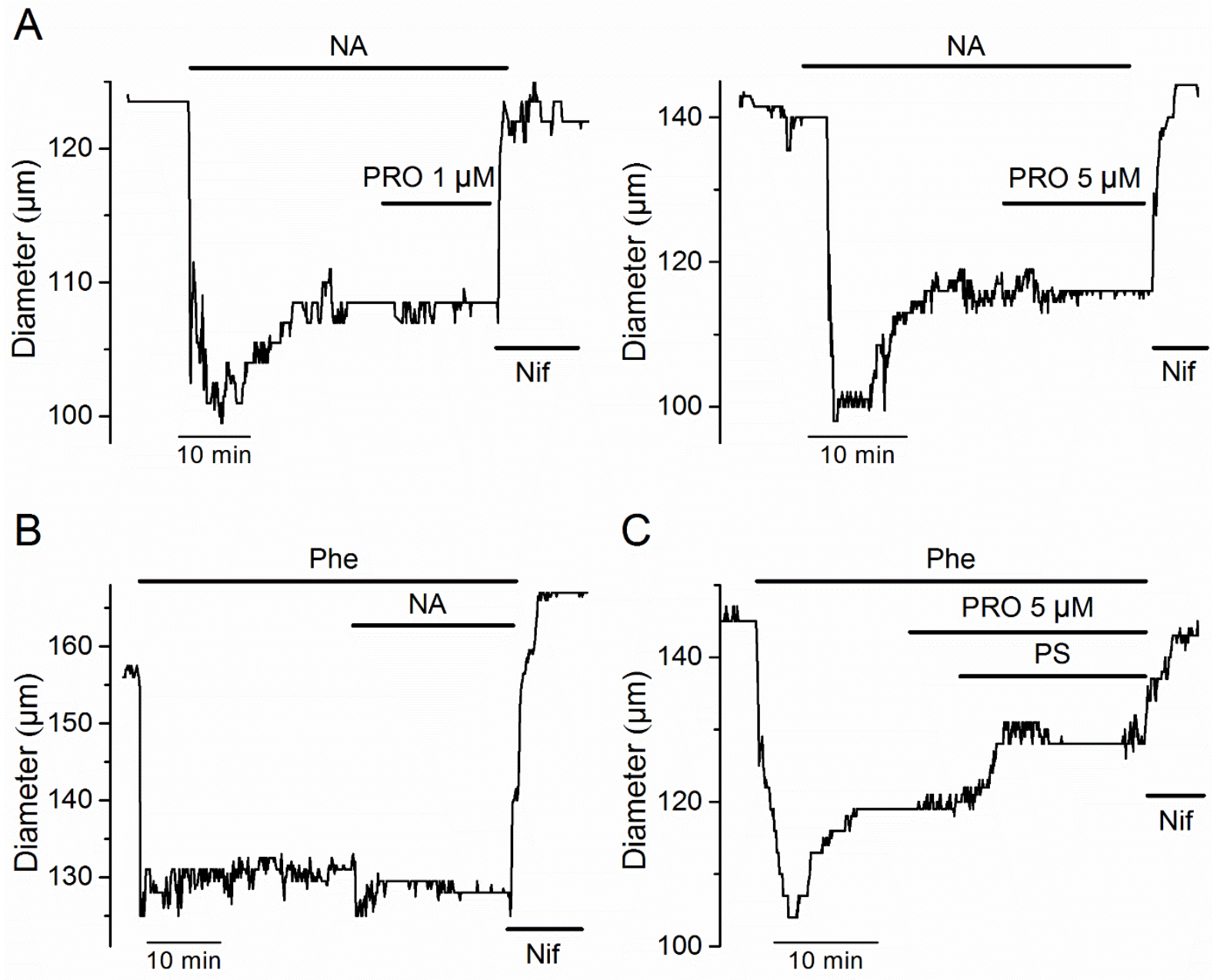

Figure 4.16. Sympathetic perivascular nerves are not involved in TRPM3-induced vasodilation of mesenteric arteries. (A) Representative example of the effect of noradrenaline (NA, $20 \mu \mathrm{M}$ ) on a $\mathrm{c} 57 \mathrm{bl} / 6 \mathrm{j}$ mesentery artery in the presence of the $\beta$-adrenoreceptor blocker propranolol (PRO, $1 \mu \mathrm{M}$ in the left panel and $5 \mu \mathrm{M}$ in the right panel). (B) Effect of $10 \mu \mathrm{M}$ noradrenaline on $\mathrm{c} 57 \mathrm{bl} / 6 \mathrm{j}$ arteries precontracted with phenylephrine (Phe, $20 \mu \mathrm{M})$. (C) Effect of PS $(10 \mu \mathrm{M})$ in the presence of propranolol $(5 \mu \mathrm{M})$. Nifedipine $(10 \mu \mathrm{M})$ was applied at the end of each experiment. 


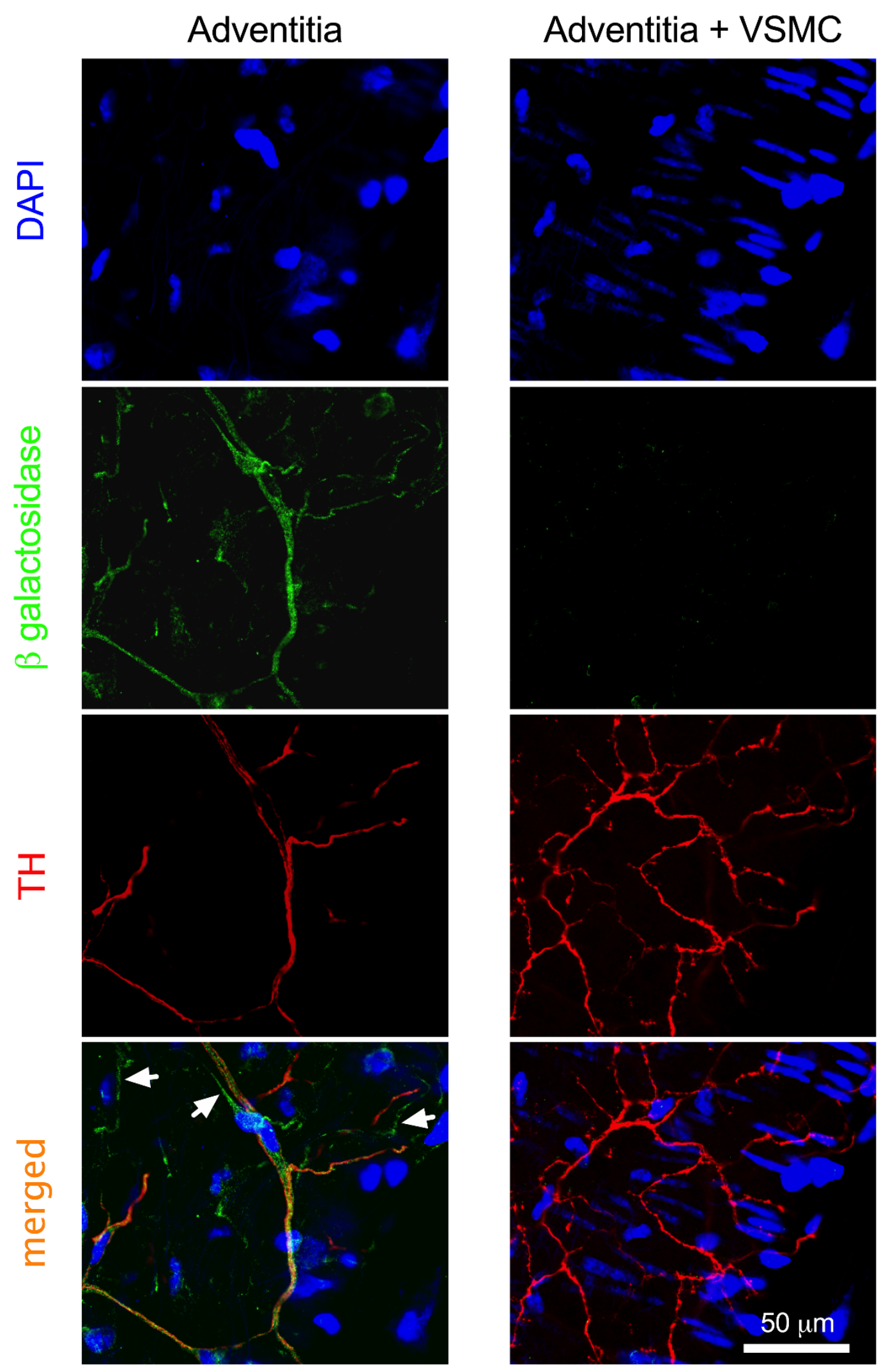

Figure 4.17. Confocal images of intact Trpm3 KO mouse mesenteric arteries at the level of the adventitial (left panels) and closer to the medial (right panels) layers, labeled with nuclear DAPI staining (blue), $\beta$-galactosidase (green) and Tyrosine hydroxylase (red). Images are representative of at least 3 independent experiments. The position was set according to nuclei morphology, 40X. 


\section{Role of TRPA1 and TRPM8 channels in intrinsic vascular responses to cold}

Lucía Alonso-Carbajo ${ }^{1,2}$, José Ramón López-López ${ }^{2}$, María Teresa Pérez-García ${ }^{2}$ and Karel Talavera $^{1}$

${ }^{1}$ Department of Cellular and Molecular Medicine, Laboratory of Ion Channel Research, KU Leuven; VIB Center for Brain \& Disease Research, Leuven Belgium.

${ }^{2}$ Departamento de Bioquímica y Biología Molecular y Fisiología, Instituto de Biología y Genética Molecular, Universidad de Valladolid y CSIC, Valladolid, Spain.

Manuscript in preparation; Lucía Alonso-Carbajo performed all the experiments and analyzed all data. 


\subsection{TRPA1 and TRPM8 channels are involved in intrinsic vascular responses to cold}

It is well known that cold induces vasoconstriction in skin blood vessels as a protective response against heat loss. This phenomenon is thought to be mediated by an efferent physiological reflex in response to the activation of cold-sensitive afferent nerves [213]. The mechanism underlying this process has been described to be mediated by activation of TRPA1 and TRPM8 channels in sensory neurons. On the other hand, the control of regional circulations and the local vasomotor responses to thermal stimulus has also been proposed by Bowell [214] ; however, the mechanisms underlying and the molecular players involved in these responses are still unclear.

In order to determine whether TRPA1 and TRPM8 channels are involved in the intrinsic coldinduced vascular response, we performed pressure myography experiments in isolated plantar arteries from male mice. Following baseline measurements under physiological solution with $1 \mu \mathrm{M}$ Phe at $37{ }^{\circ} \mathrm{C}$, the arteries were exposed to cold solution $\left(10^{\circ} \mathrm{C}\right.$ for at least $5 \mathrm{~min})$ and finally to the L-type $\mathrm{Ca}^{2+}$ channel blocker nifepidine $(10 \mu \mathrm{M})$ in order to determine the maximum arterial diameter.

We found that cold solution $\left(10^{\circ} \mathrm{C}\right)$ induced a contraction in endothelium-denuded plantar arteries dissected from c57bl/6j mice $(29 \pm 2.8 \%, \mathrm{n}=6$; Figure $5.1 \mathrm{~A}$ and $\mathrm{C})$. In previous experiments, if the myogenic tone was not induced to the arteries, they did not response to cold temperature. Therefore, all the arteries were precontrated with $1 \mu \mathrm{M}$ Phe before starting the experiment. In order to explore if the cold-induced effect was only restricted to cutaneous vascular beds, we exposed internal arteries of the body such as mesenteric arteries to cold solution and we observed that in contrast to plantar arteries, the vasoconstrictor responses by low temperatures were not observed in these arteries $(1.6 \pm 1.02 \%$, Figure $5.1 B$ and $C)$. 
A

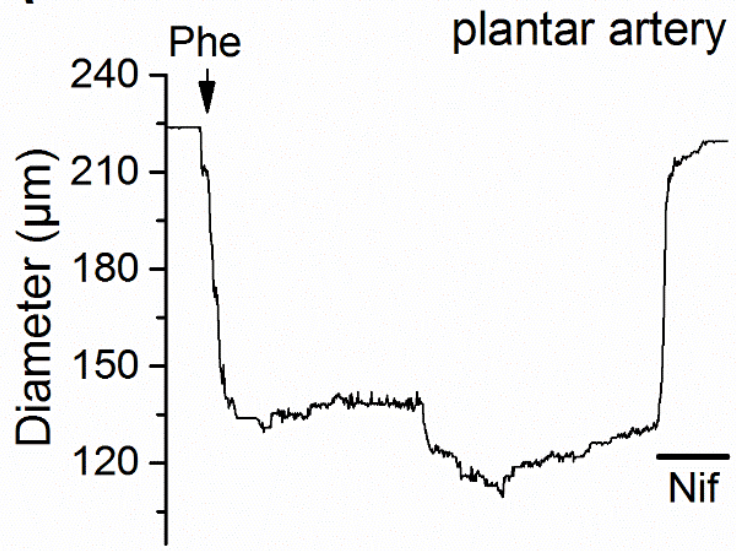

B
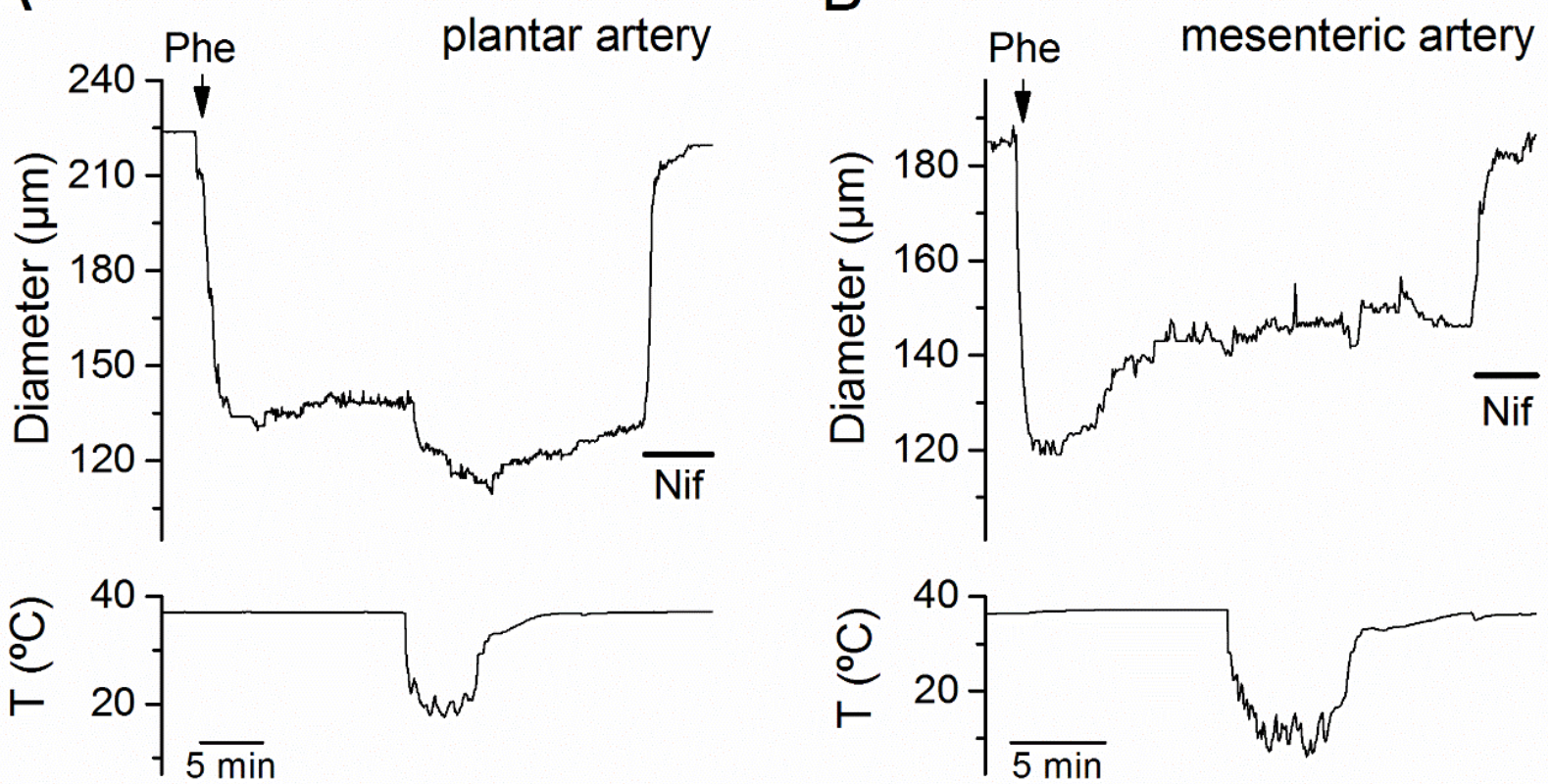

C

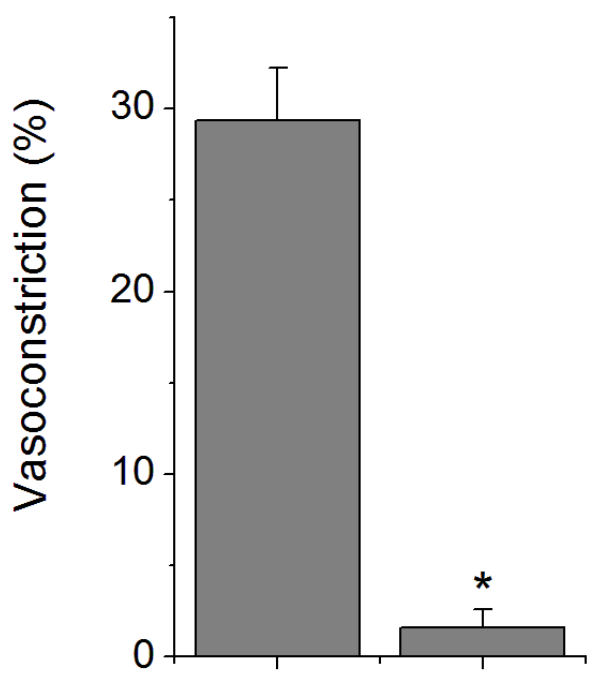

Figure 5.1. Vascular response to cold in different types of vascular beds. (A) Representative example of a precontracted plantar artery which is modulated by low temperatures $\left(18{ }^{\circ} \mathrm{C}\right)$. (B) Example of a pre-contracted mesenteric artery showing the lack of reaction against cold stimulation $\left(10^{\circ} \mathrm{C}\right) .(\mathrm{C})$ Mean values of the effect in response to cold in plantar arteries $(n=6)$ and mesenteric arteries from c57bl/6j $\left(n=4 ;{ }^{*} p<0.005\right.$, one-way ANOVA).

In plantar arteries, the maximum vasoconstriction was observed at 1 to 4 min following local cooling and was determined as the percentage decrease in diameter from the baseline before cold and the maximum diameter of the artery using Eq. 3.4. Exposure of plantar arteries from c57bl/6j mice to $10{ }^{\circ} \mathrm{C}$ revealed a significantly vasoconstriction $(28 \pm 3.01 \%, \mathrm{n}=9$; Figure $5.2 \mathrm{~A}$ and $D$ ) that was significantly less in plantar arteries dissected from Trpa1 KO mice (11.04 \pm $2.78 \%, \mathrm{n}=11,{ }^{*} \mathrm{p}<0.005$; Figure $5.2 B$ and $\left.D\right)$. 

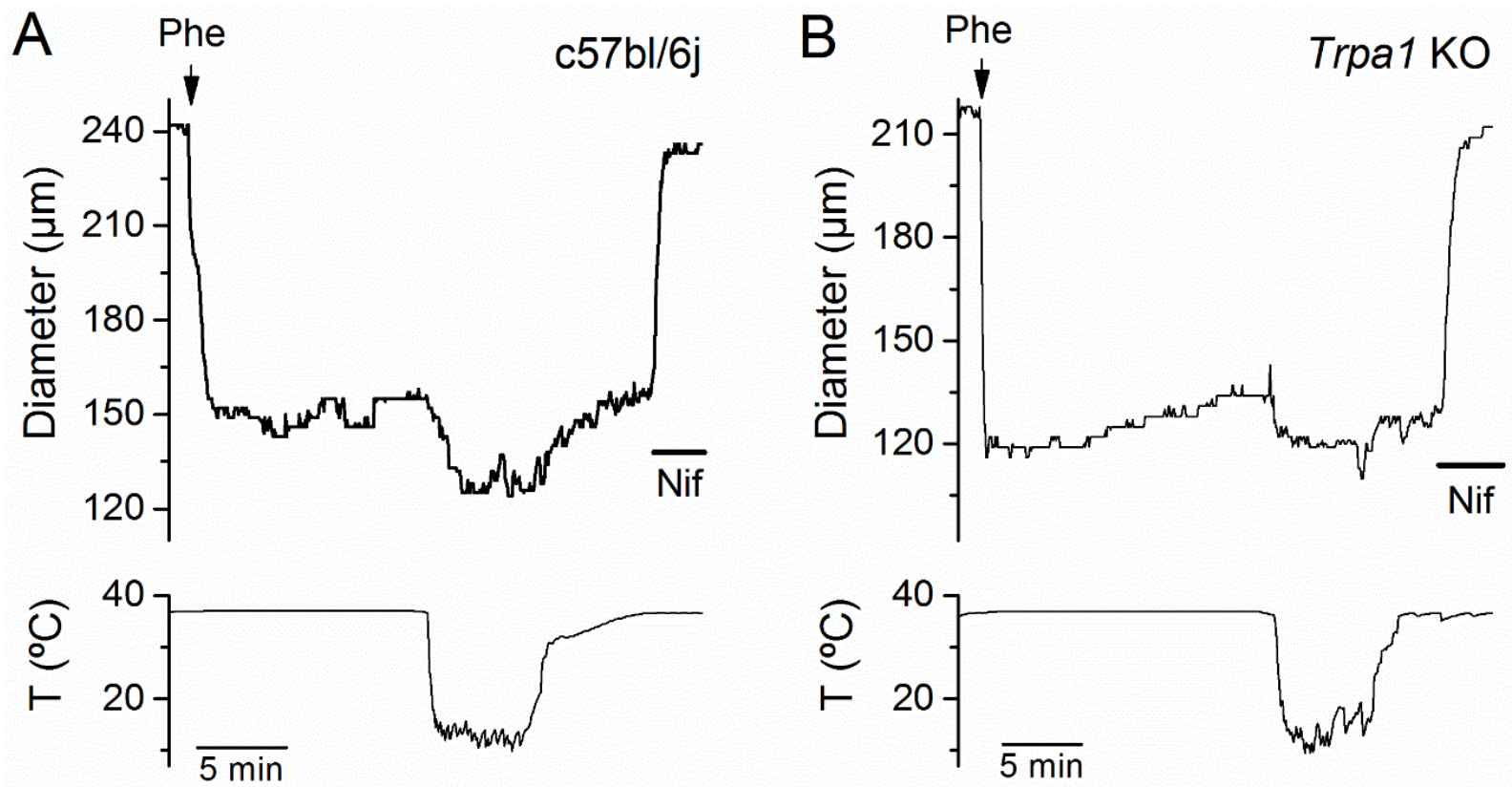

C
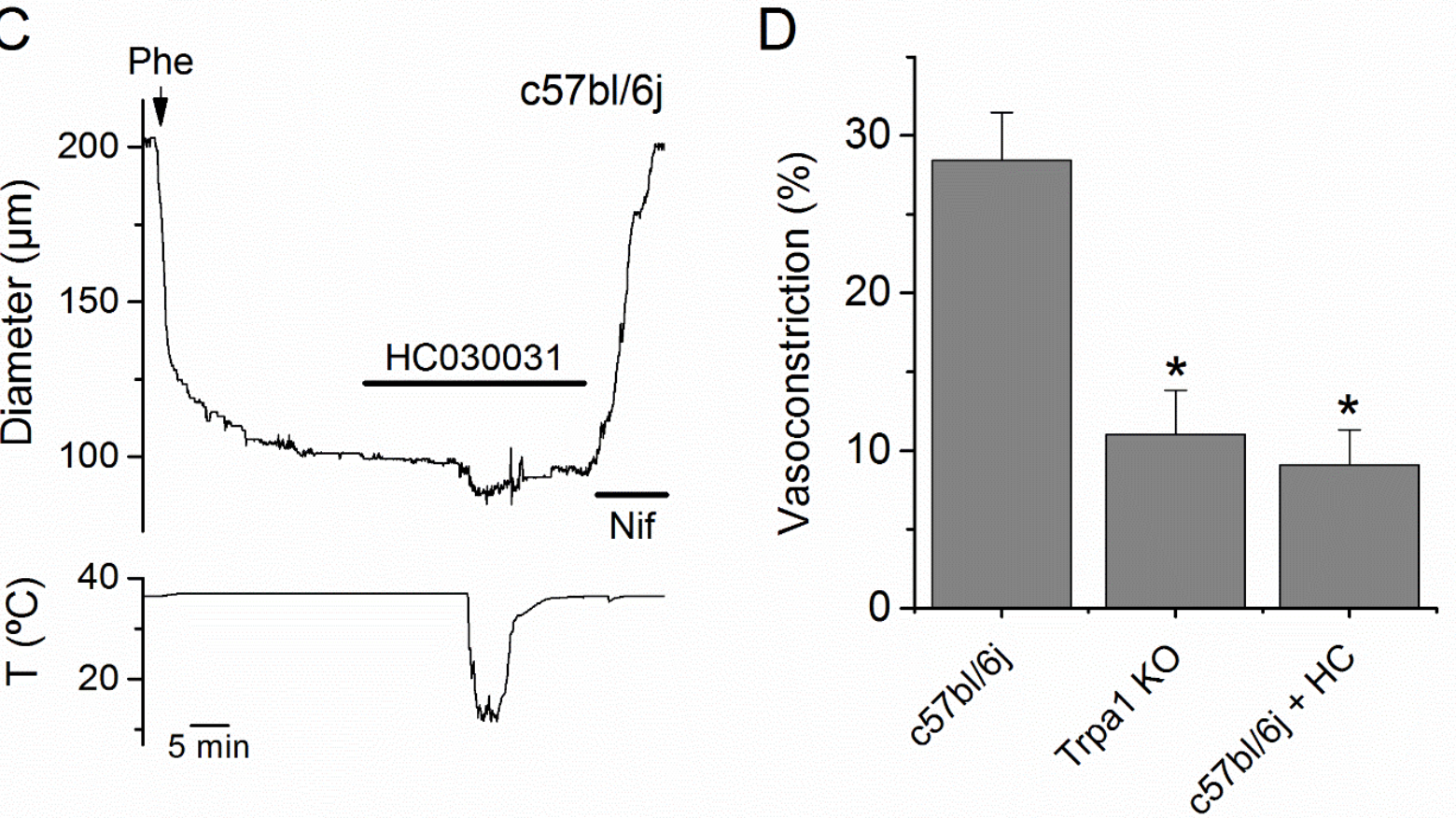

Figure 5.2. TRPA 1 channel as a cold sensor in plantar artery. $(A)$ In c57bl/6j plantar arteries, cold stimulation induces vasoconstriction. $(B)$ Low temperature does not produce any effect on Trpa1 KO plantar arteries. (C) Reduced effect of cold in the presence of the TRPA1 blocker HC030031 (10 $\mu \mathrm{M})$. (D) Mean values of the effect in response to cold in plantar arteries from c57bl/6j $(n=9)$, Trpa1 KO ( $=11$ ) and in the presence of HC030031 ( $n=6 ;{ }^{*} p<0.005$, one-way ANOVA).

The responses of plantar arteries to cold were also significantly smaller in c57bl/6j mice pretreated with the TRPA1 antagonist HC030031 $\left(9 \pm 2.23 \%, n=6,{ }^{*} p<0.005\right.$; Figure $5.2 C$ and $D)$, while removal of the inhibitor cold-induced response was restored to control ( $31 \pm 2.02 \%$, 
$n=6)$. Thus, TRPA1 channels partially mediates the vasoconstrictor response of the local coldinduced vascular response.

A
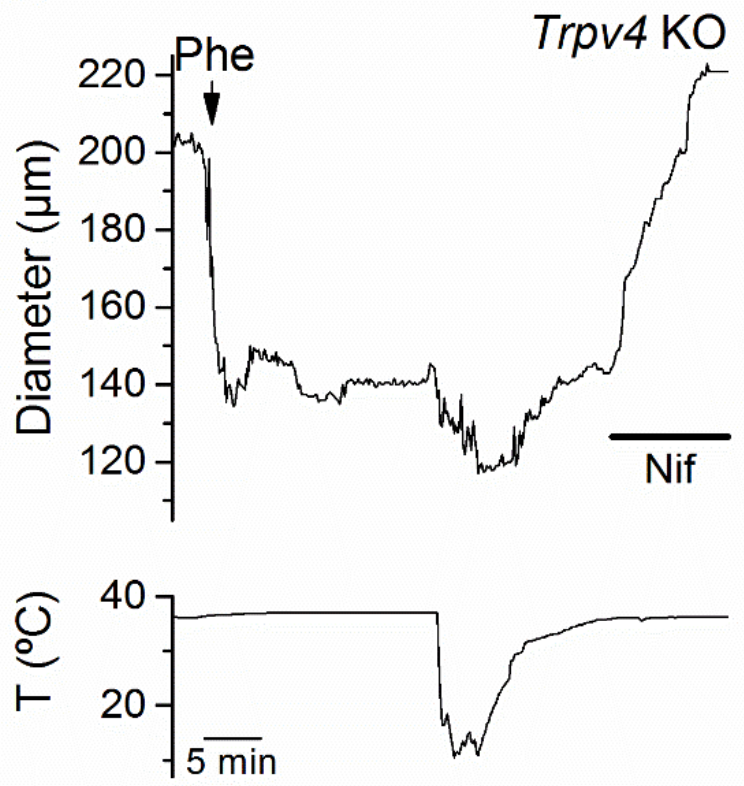

C
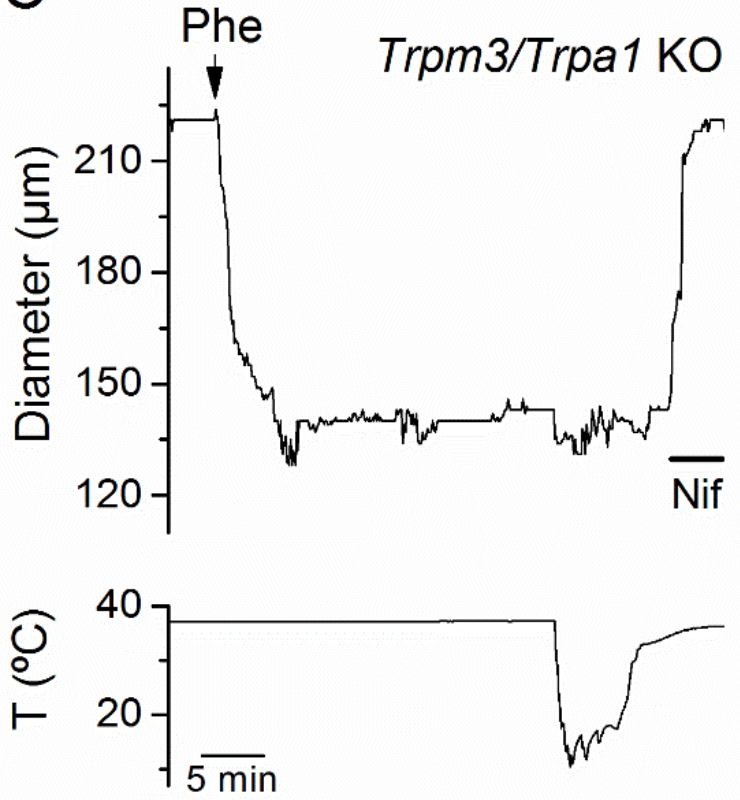

B
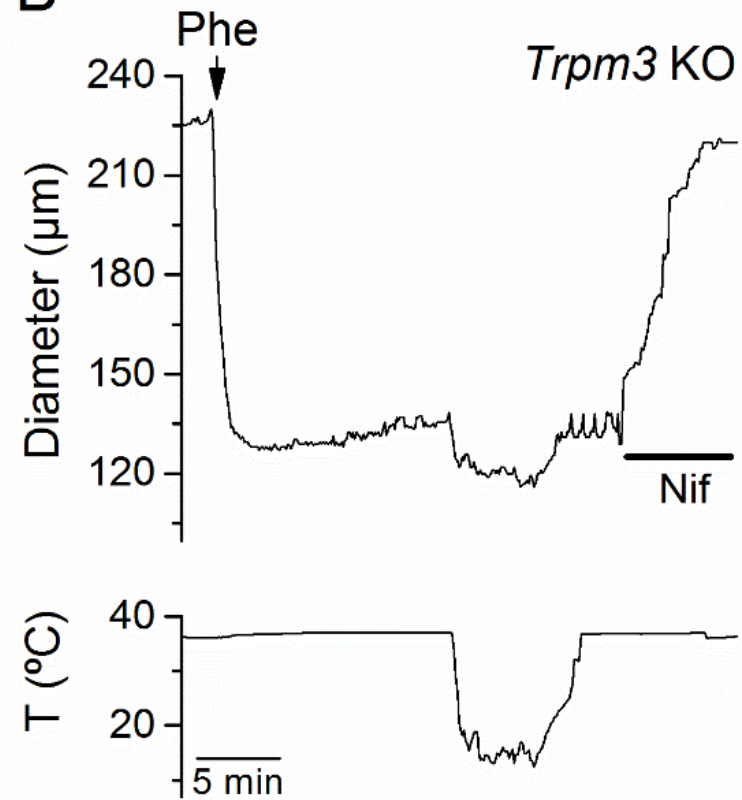

D
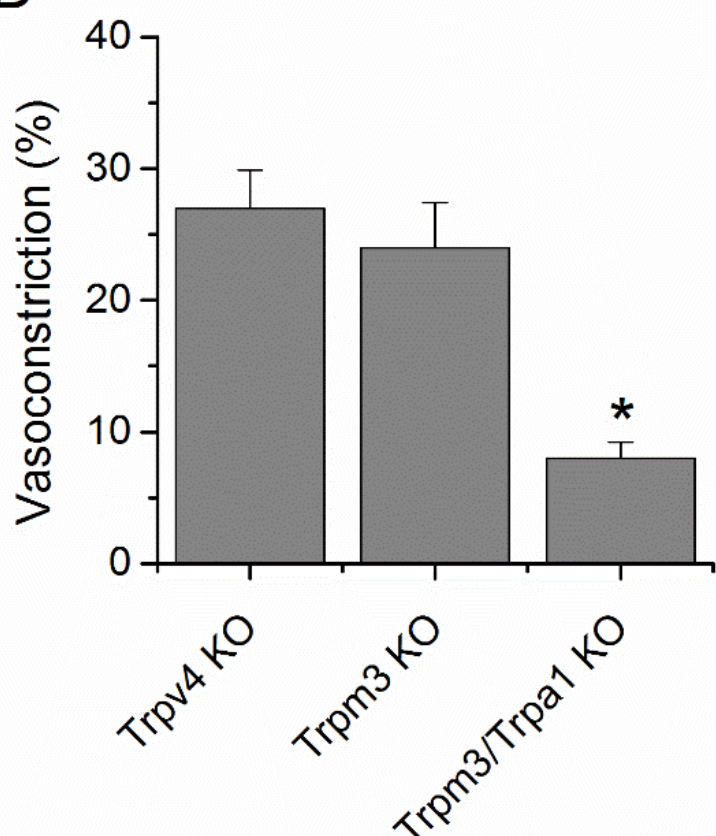

Figure 5.3. Cold-induced vascular responses are not dependent on TRPV4 or TRPM3. (A) Cold stimulation induces vasoconstriction in Trpv4 KO (A) and Trpm3 KO (B) plantar arteries. (C) Reduced effect of cold in Trpm3/Trpa1 KO plantar arteries. (D) Mean values of the effect in response to cold in plantar arteries from Trpv4 KO $(n=4), \operatorname{Trpm} 3 \mathrm{KO}(\mathrm{n}=4)$ and Trpm3/Trpa1 KO $\left(\mathrm{n}=7 ;{ }^{*} \mathrm{p}<0.005\right)$. 
On the other hand, gene deletion of Trpv4 or Trpm3 (two predicted warm temperature sensors from 25 to $\left.34{ }^{\circ} \mathrm{C}[62,215]\right)$ did not alter cold-induced vascular responses with contraction amplitudes similar to that of c57bl/6j arteries $(27 \pm 2.9 \%, \mathrm{n}=4$ for Trpv4 KO, $24 \pm$ 3.4\%, $\mathrm{n}=4$ for Trpm3 KO; Figure 5.3A, B and D). Finally, experiments in plantar arteries from Trpm3/Trpa1 KO mice yielded similar results as in Trpa1 KO mice (8 $\pm 1.2 \%, \mathrm{n}=7$; Figure 5.3C and $D$ ) confirming the essential role of TRPA1 in the cold response.
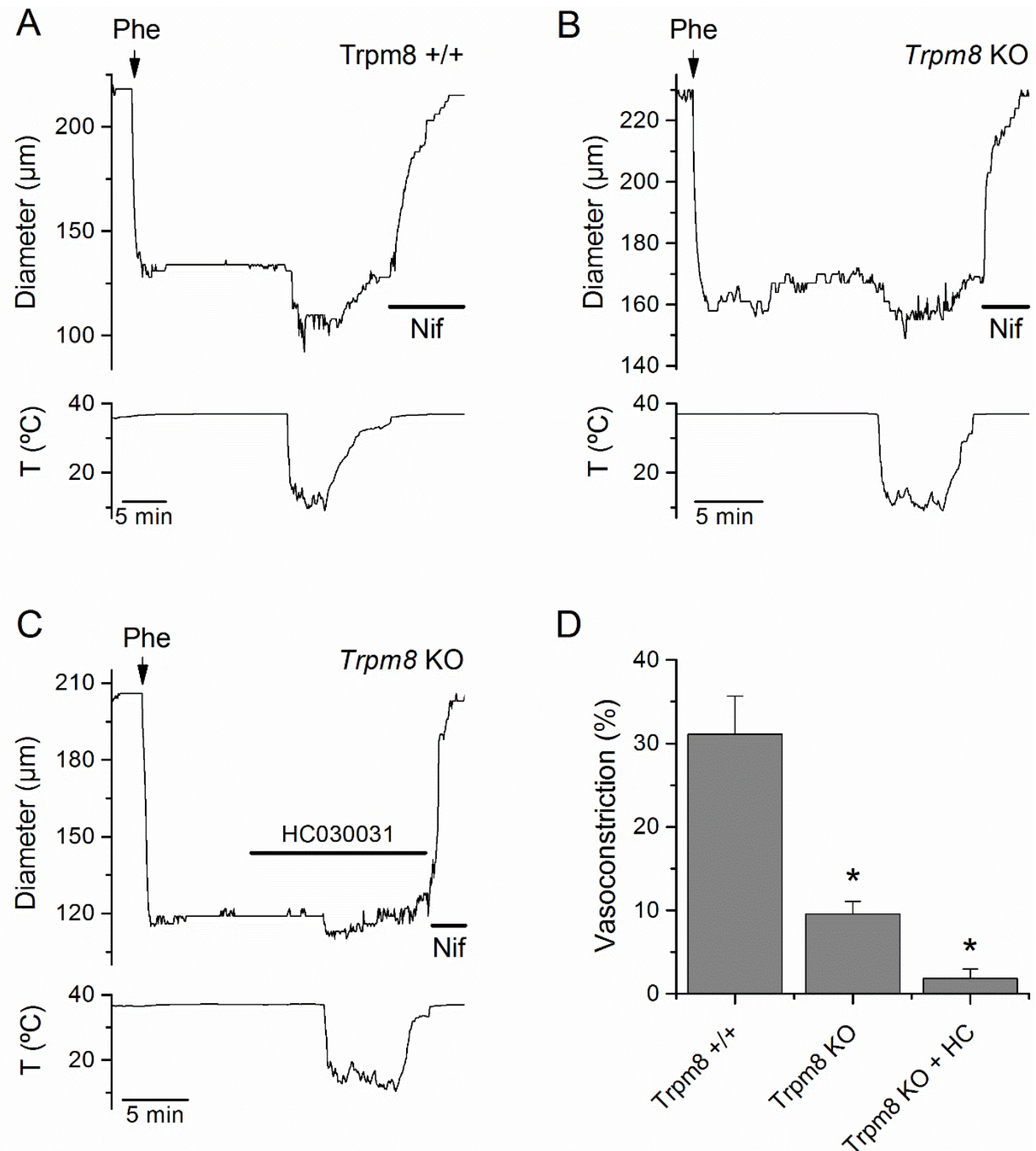

Figure 5.4. Role of TRPM 8 in the effects of cold. (A) Plantar artery from Trpm8 mice shows vasoconstriction in response to cold. (B) Cold-induced vasoconstriction is reduced in Trpm8 $\mathrm{KO}$ arteries. (C) The cold-induced effect is completely abolished in Trpm8 KO plantar arteries in the presence of $\mathrm{HC030031}(10 \mu \mathrm{M})$. (D) Mean values of the effect in response to cold in plantar arteries from Trpm $8+/+(n=6), \operatorname{Trpm} 8 \mathrm{KO}(n=6)$ and Trpm8 KO in the presence of HC030031 $\left(n=5 ;{ }^{*} p<0.005\right.$, one-way ANOVA). 
TRPM8 rather than TRPA1 is considered to have an important role in cold sensation. The responses of Trpm8 KO plantar arteries were reduced in comparison to those of TRPM $8+/+$ arteries $(31 \pm 3.9 \%, \mathrm{n}=6$ for Trpm8 +/+ and $10 \pm 1.5 \%, \mathrm{n}=6$ for Trpm8 KO; Figure 5.4A, $B$ and D). In addition, application of HC030031 to plantar arteries from Trpm8 KO mice virtually abolished the responses to cold $(2 \pm 1.1 \%, \mathrm{n}=5$; Figure $5.4 \mathrm{C}$ and $\mathrm{D})$ indicating that the combined, additive activation of TRPA1 and TRPM 8 channels accounts for the whole coldinduced vasoconstrictor response.

\subsection{Implication of the perivascular innervation in intrinsic vascular responses to cold}

Next, we explored the mechanism(s) through which TRPA1 and TRPM8 mediate cold-induced vascular response. Neither TRPA1 nor TRPM8 channels could be detected in VSMC from plantar arteries, suggesting that the response is mediated by activation of these channels in perivascular sensory or sympathetic nerves via neuropeptides or catecholamine release, respectively. If these cold-sensitive channels were present in sensory nerves, their activation may lead to vasodilation. We observed that in c57bl/6j plantar arteries cold-induced vasoconstriction was potentiated in the presence of the CGRP receptor inhibitor BIBN (40 \pm $4.26 \%, \mathrm{n}=8$; Figure 5.5A and $D$ ), consistent with the idea of a subpopulation of these channels being expressed in sensory terminals.

On the other hand, if the TRPA1 or TRPM8 channels participating in this response were also expressed in sympathetic terminals, their activation is expected to produce vasoconstriction. Indeed, we found that guanethidine-induced depletion $(10 \mu \mathrm{M})$ of the peripheral sympathetic nerves caused a significant vasodilation on the cold-induced response $(9 \pm 2.6 \%, \mathrm{n}=7$; Figure $5.5 B$ and $D)$. No effect on vessel diameter was elicited by cold when arteries were incubated with both guanethidine $(10 \mu \mathrm{M})$ and BIBN $4096(1 \mu \mathrm{M})(2 \pm 1.1 \%, \mathrm{n}=5$; Figure 5.5C and D). These data indicate that cold activates TRPA1 and/or TRPM8 channels expressed in sensory and sympathetic neurons. The cold-induced vascular response in the presence of BIBN $(1 \mu \mathrm{M})$ and/or guanethidine $(10 \mu \mathrm{M})$ in Trpm8 +/+ mice did not differ from the one found in c57bl/6j (Figure 5.6). 
A
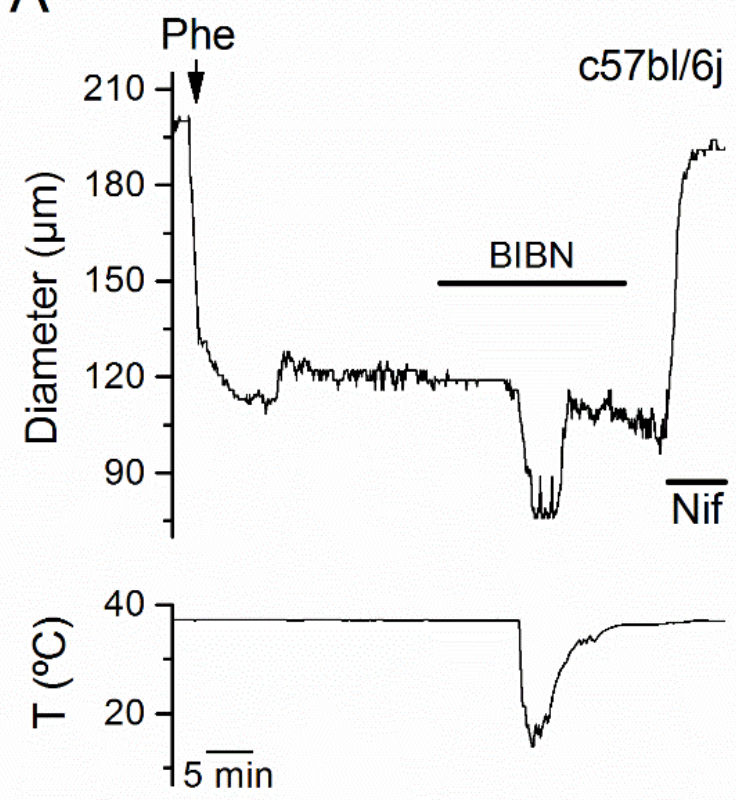

C
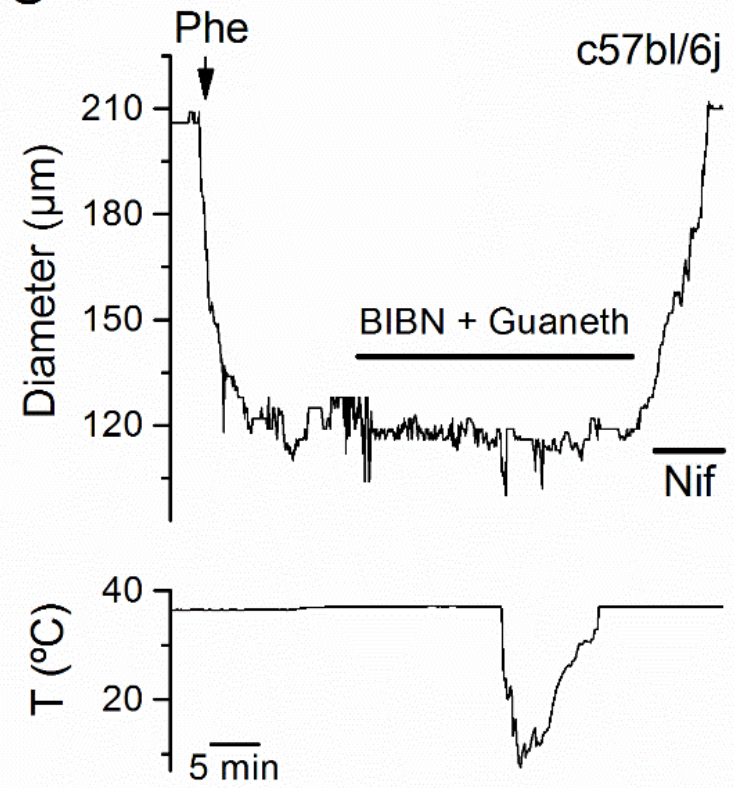

B
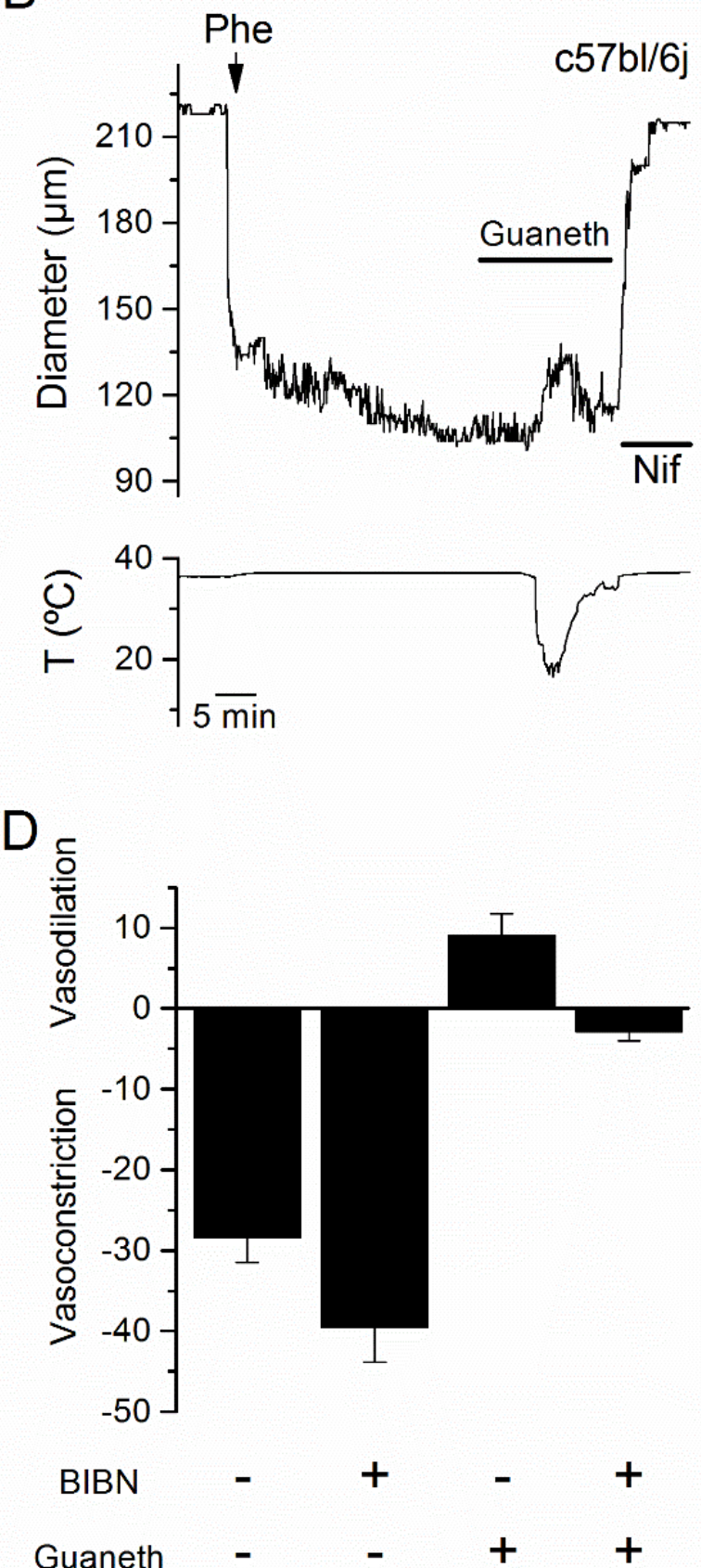

Figure 5.5. Cold-induced vascular response involves sensory and sympathetic nerves activation. (A) Cold induces relaxation in the presence of NA release blocker guanethidine (10 $\mu \mathrm{M})$. (B) Colddependent vasoconstriction is increased in the presence of CGRP antagonist (BIBN) $(1 \mu M)$. (C) The mixture of both blockers almost completely abolished the cold effect. (D) Mean values of the effect in response to cold in plantar arteries from $\operatorname{Trpm} 8+/+(n=6)$, in the presence of $\operatorname{BIBN}(n=5)$, guanethidine $(n=4)$ and both blockers $(n=5)$. 
A
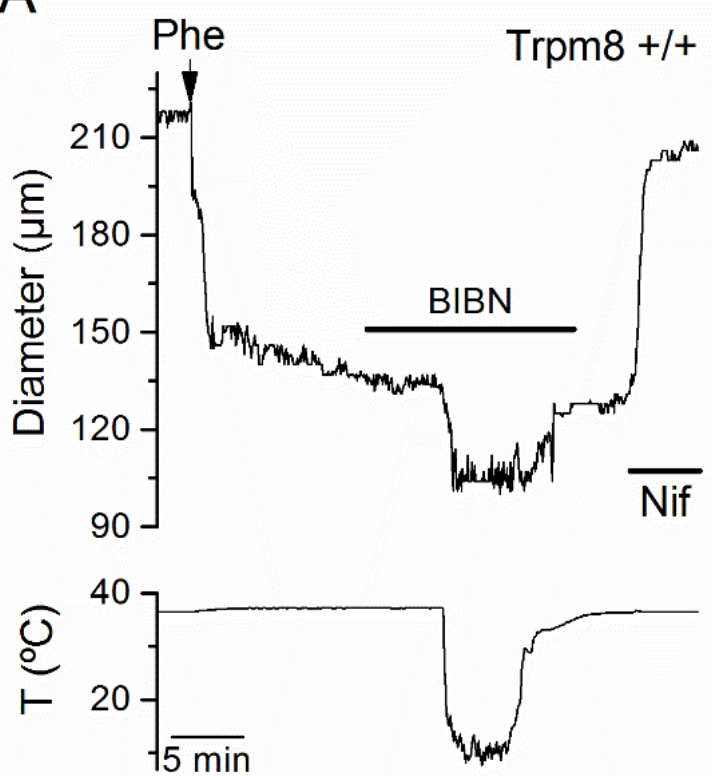

C
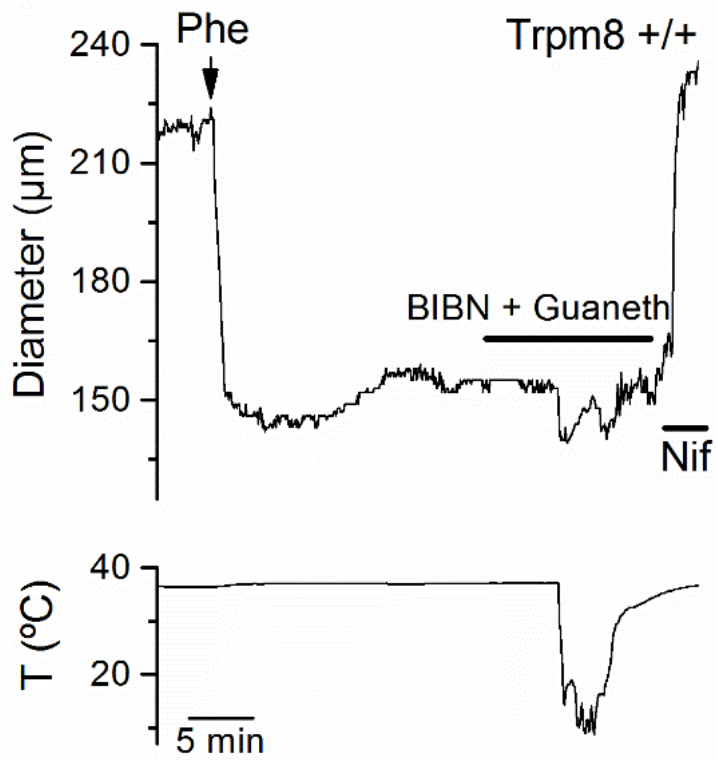

B
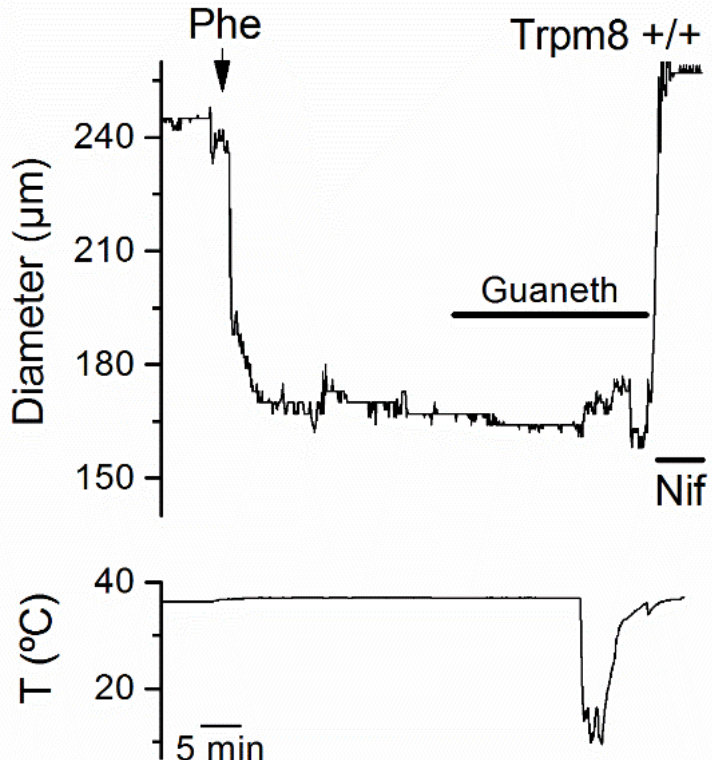

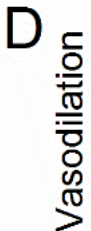

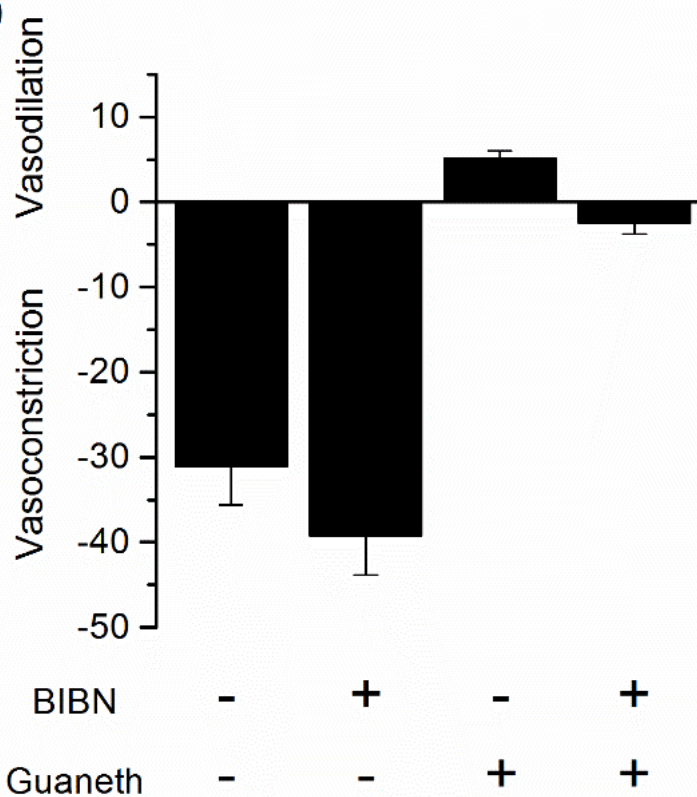

Figure 5.6. Cold-induced vascular response also involves sensory and sympathetic terminals in Trpm8 $+/+$ mice. (A) Cold induces relaxation in the presence of NA release blocker guanethidine $(10 \mu \mathrm{M})$. (B) Cold-dependent vasoconstriction is increased in the presence of CGRP antagonist (BIBN, $10 \mu \mathrm{M})$. (C) The mixture of both blockers inhibits the cold effect. $(D)$ Mean values of the effect in response to cold in plantar arteries from $\operatorname{Trpm} 8+/+(n=6)$, in the presence of BIBN $(n=5)$, guanethidine $(n=4)$ and both blockers $(n=5)$.

Furthermore, we confirmed that TRPA1 and TRPM8 channels are present in sympathetic neurons by detecting mRNA of both channels in isolated superior cervical sympathetic ganglia (SCG) from c57bl/6j mice (Figure 5.7A). We could also confirm the presence of perivascular 
sympathetic nerves in confocal images of plantar arteries labelled with an anti-TH antibody (Figure 5.7B). However, we could not detect mRNA expression of TRPA1 and TRPM8 channels in isolated VSMC from plantar arteries.

These data suggest that cold has a dual action, a modest vasodilatory response through activation of TRPA1 and TRPM8 channels in sensory nerve endings, and a dominant, more potent vasoconstrictor response via the activation of TRPA1 and TRPM8 channels in sympathetic nerve endings. Our results represent the first evidence for an intrinsic response to cold in cutaneous arteries and for the functional role of sensory TRP channels in sympathetic nerve fibres.

A

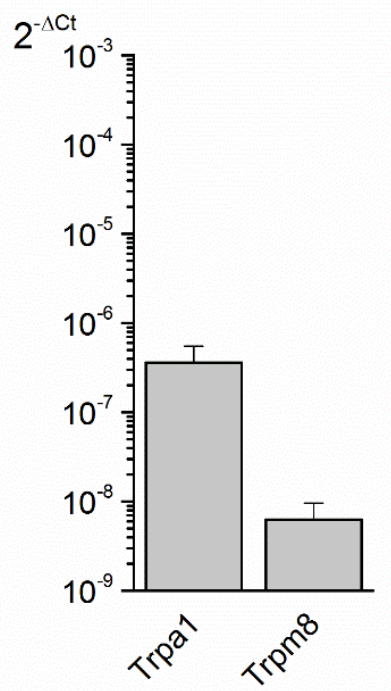

B Tyrosine Hydroxylase

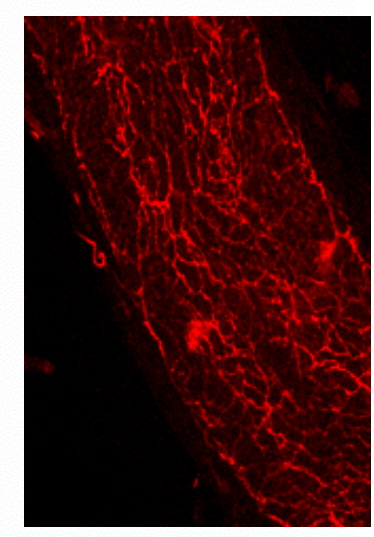

Figure 5.7. Trpa1 and Trpm8 mRNA are present in superior cervical sympathetic ganglia from $\mathrm{c} 57 \mathrm{bl} / 6 \mathrm{j}$ plantar arteries. (A) Expression of TRPA1 and TRPM8 channels in cervical sympathetic neurons (B) Confocal images from c57bl/6j plantar artery demonstrate the presence of sympathetic fibres by the localization of Tyrosine hydroxylase, 20x. 


\section{Discussion, General Conclusions and Future Perspectives}




\subsection{Discussion}

The deregulation of the vascular function under external stimuli is an essential process that can lead to cause severe vascular disorders and even sudden death. Resistance arteries can influence intracellular $\mathrm{Ca}^{2+}$ signaling in response to chemical and thermal challenges resulting in the modulation of vascular tone. Recent findings indicate that sensory TRP proteins play important roles in the arterial function due to their chemo- and thermosensory properties, but their actual relevance to vascular physiology remains fully unknown. Here, we provide evidence that sensory TRP channels are highly expressed in perivascular nerves and can regulate the vascular tone of resistance arteries in response to external stimuli.

\subsubsection{Activation of the cation channel TRPM3 in perivascular nerves induces vasodilation of resistance arteries}

TRP channels have been found in all cell types relevant for vascular function. Endothelial sensory TRP channels regulate angiogenesis and vascular tone and permeability, whereas in VSMC they are implicated in the regulation of contraction and proliferation [216]. Sensory TRP channels are also expressed in perivascular cells, such as TRPV4 in astrocytes and TRPV1 and TRPA1 in sensory neurons [217]. Therefore, we investigated the expression and functional role of the emerging sensory TRPM 3 channel in resistance arteries.

Although we found Trpm3 mRNA in VSMC and adventitia layer, our anatomical and functional data are consistent with an expression of TRPM3 protein restricted to perivascular nerve endings. A previous report showed functional expression of TRPM3 channels in proliferating human VSMC and in freshly isolated mouse aortic myocytes [128], but we did not find responses to PS in freshly isolated mouse mesenteric myocytes. Whether these differences may reflect vascular bed-dependent functional expression of TRPM3 requires further investigation. Our qPCR studies show that Trpm3 mRNA expression is almost 40 times higher in aorta than in mesenteric arteries. This is in line with a functional expression of TRPM3 in aortic VSMC, whose activation leads to $\mathrm{Ca}^{2+}$ entry and vasoconstriction [128].

In contrast to these previous observations in aorta [128], we found that PS induced vasodilation of mesenteric arteries. The maximal effect of this compound was comparable to the maximal dilation induced by nifedipine or $\mathrm{Ca}^{2+}$ free solution. A previous report showed that genetic ablation of Trpm3 abolished CGRP release from skin and insulin secretion from 
isolated pancreatic islets in response to $100 \mu \mathrm{M}$ PS [135]. However, we found that PS induced dilation via two components, the high $E C_{50}$ component still present in arteries isolated from Trpm3 KO mice. Our results show that PS has TRPM3-independent effects in mesenteric arteries at concentrations higher than $10 \mu \mathrm{M}$. This compound has been shown to act on other targets, such as the gamma-aminobutyric acid receptor $\mathrm{A}$ and the $\mathrm{N}$-methyl-D-aspartate receptor [133], but it is not yet clear whether these bare any relevance in mesenteric arteries. Of note, the values of the $E C_{50}$ and Hill coefficient corresponding to the TRPM3-independent PS responses were different in $\mathrm{c57bl} / 6 \mathrm{j}$ and Trpm3 $\mathrm{KO}$ arteries. This may be due to the fact that in $\mathrm{c} 57 \mathrm{bl} / 6 \mathrm{j}$ arteries the TRPM3-dependent processes may affect the TRPM3-independent vasodilation observed at higher PS concentrations. The nonlinear and integrative character of the vasodilation process may preclude that the effects of individual PS-dependent components add up in a simple arithmetic way. Further assessment of this observation will be possible once the mechanism underlying the latter component is clarified. Nevertheless, the data fitting results indicate that the TRPM3-dependent component contributes to an important fraction of the total PS induced dilation of c57bl/6j arteries ( $60 \%)$, and its occurrence in the lower concentration range suggest that it is the most relevant component in physiological conditions.

In regard to the mechanism underlying TRPM3-dependent vasodilation, the overlap we observed between the PS dose response curves obtained for Trpm3 KO arteries and for c57bl/6j arteries in the presence of the CGRP receptor inhibitor suggests that PS-induced CGRP effects are fully mediated by TRPM3. Previous studies demonstrated CGRP release upon TRP channel activation in mouse trachea [218] and hind paw skin [135]. However, we were unable to detect CGRP release from mesenteric using similar experimental procedures (data not shown), most likely because our preparation is between 200 and 2000 fold smaller than the trachea and skin ones, respectively.

The mechanisms proposed for the vasodilating effects of CGRP include an endotheliumdependent component, whereby activation of the endothelial CGRP receptor results in a rise in CAMP, NO production and guanylate cyclase-mediated vasodilation [219]. In addition, there are two effects mediated by AC and PKA stimulation in VSMC: activation of $\mathrm{K}^{+}$channels and stimulation of myosin light chain phosphatase $[19,220]$. Our data are consistent with all these mechanisms, as we found that the responses to PS were enhanced in the presence of endothelium and were partially inhibited by a cocktail of $\mathrm{K}^{+}$channel blockers. 
Furthermore, we could exclude a possible contribution of sympathetic fibres to the PS induced vasodilation mechanisms. Our data exclude both the functional contribution of $\beta_{2^{-}}$ adrenoreceptors to the sympathetic response and the involvement of these receptors in the vasodilation induced by PS application, as no differences were observed when we applied the $\beta$-blocker propranolol. Moreover, the absence of colocalization of TH and $\beta$-gal in the nerve fibres innervating the media layer indicates a lack of expression of TRPM3 in sympathetic nerve endings.

Notably, it was previously shown that CGRP-containing fibres and TH-containing fibres can be found in close proximity of each other in mouse mesenteric arteries, and that the former run further away from the VSMC [221], as we found for TRPM3- and $\beta$-gal-expressing nerves.

Notably, the vasodilation we report here is the most sensitive TRPM3-mediated tissue response to PS reported so far. Previous studies showed TRPM3-dependent effects of PS in the range of tens to hundreds of micromolar, e.g., insulin release from pancreatic islets [130], CGRP release from mouse skin preparations [135] and rat ductus arteriosus contraction [222]. In contrast, we found significant TRPM3-dependent vasodilation with an $E C_{50}$ of $7.7 \mu \mathrm{M}$ in intact arteries. This value is similar to those reported for stimulation of TRPM3 currents in vitro $(5-23 \mu \mathrm{M})[124,134]$. Most likely, this is a consequence of the technical approach in myography experiments since PS has direct access to the TRPM3-expressing nerve endings, which contrasts with other preparations in which structural barriers are expected to interfere with PS diffusion. This suggests the mesenteric artery preparation as very instrumental for studies on TRPM3 modulation and pharmacology, including the testing of the specificity of previously described channel inhibitors [129, 139], in a close-to-physiological context. In addition, our results shed light on the long-standing question of whether PS is after all a physiological endogenous agonist of TRPM3 [130]. We observed TRPM3-dependent vasodilation induced by PS in the low micromolar range, which matches PS concentrations that may be present in living tissues [130]. The physiological or pathological contexts in which PS reaches such concentrations in mesenteric tissue remain, however, elusive.

The idea that TRPM3 activation induces vasodilation in mesenteric arteries is further supported by the potent effect of the synthetic agonist CIM0216. However, this compound proved to be not fully specific for this channel, as it also produced vasodilation in arteries dissected from Trpm3 KO mice at concentrations above $\sim 0.1 \mu \mathrm{M}$. We argue, therefore, that CIM0216 may be used as a pharmacological tool to further investigate the role of TRPM3 in 
mesenteric arteries below these concentrations. Because this is a synthetic compound with no obvious similarity to any other known TRPM3 modulator [135], we consider that investigating the TRPM3-independent effects is interesting. Nevertheless, reporting for the first time that CIM0216 has off-target effects is of great value for future research on TRPM3 pathophysiology.

The functional expression of TRPM3 in nociceptive neurons and its contribution to noxious heat sensing [62] may suggest that the function of this channel in mesenteric arteries is the detection of noxious stimuli generated in pathological conditions. The high temperatures required for TRPM3 activation [62] strongly indicate that heat may not be a relevant stimulus of TRPM3 in mesenteric preparations. Nevertheless, it would be interesting to determine whether this channel is implicated in responses of skin resistance arteries to heat. However, in contrast to previous reports in skin and trachea [62], we show here a role of TRPM3 in sensory fibres innervating a tissue that is not directly accessible to external stimuli. This may indicate that TRPM3 functions as detector of endogenous compounds released upon tissue damage and/or metabolic deregulation. Two other sensory TRP channels, TRPV1 and TRPA1 are proposed to act as receptors of danger- and pathogen-associated molecular patterns during tissue injury and inflammatory diseases [223], by detecting acidosis, reactive oxygen and nitrogen species, electrophilic compounds and bacterial endotoxins [101, 224-226]. However, TRPV1 and TRPA1 have also been reported in VSMC and endothelial cells of resistance arteries, respectively [99, 113, 114, 227, 228]. Thus, according to our findings, TRPM3 is the only one of these sensory TRP channels exclusively functional in perivascular nerves. This suggests TRPM3 as the most specific target to trigger resistance artery vasodilation via stimulation of the perivascular sensory innervation.

We conclude that in contrast to what was previously reported in aorta [128], in mesenteric arteries TRPM3 is functionally expressed mainly in perivascular nerve endings and its activation leads to vasodilation rather than contraction (Figure 6.1). Our data is consistent with a model in which activation of TRPM3 triggers CGRP release, leading to vasodilation via endothelium-dependent and endothelium-independent pathways. We propose that, together with TRPV1 and TRPA1, TRPM3 allows mesenteric arteries to react to a wide range of damageassociated molecules, leading to vasodilation, via the common pathway of CGRP release from perivascular sensory nerve endings. 


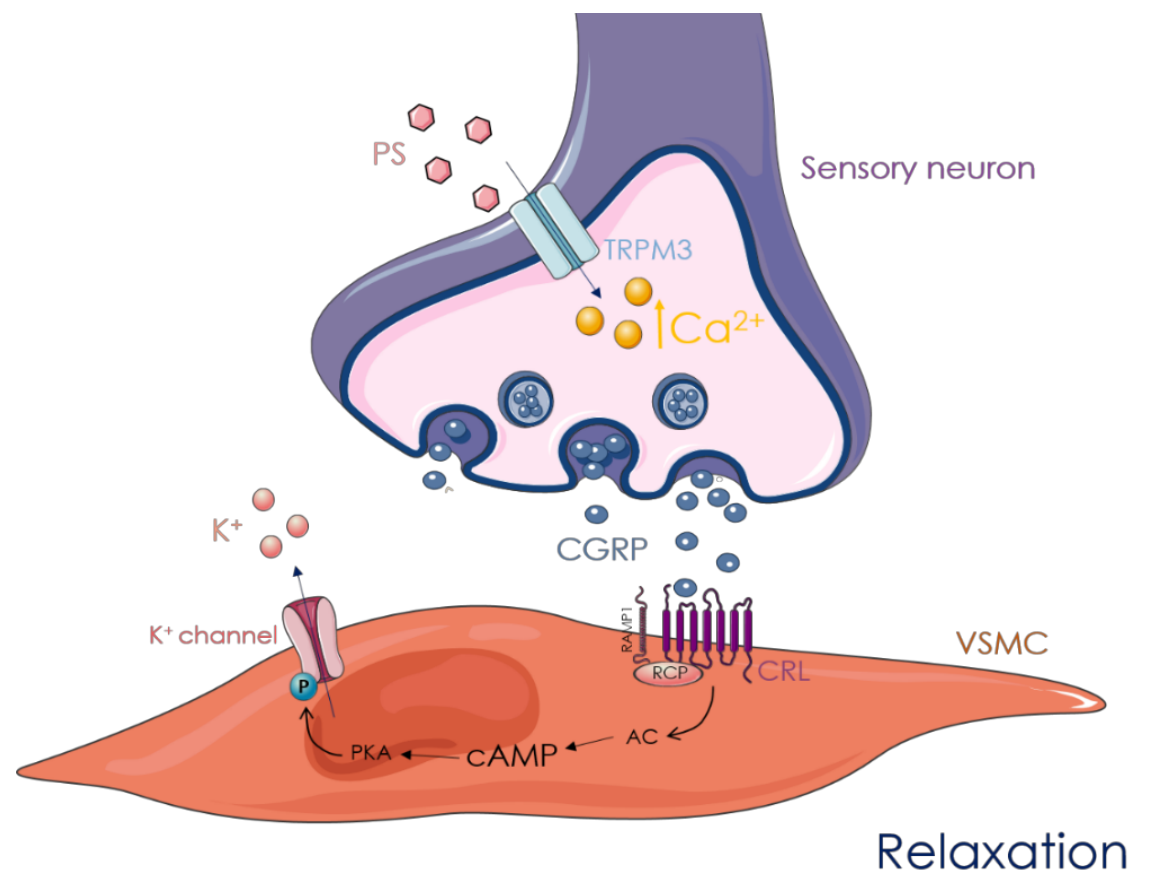

Figure 6.1. Proposed vasodilation-induced TRPM3 pathway in resistance arteries. Activation of TRPM3 channels by PS leads to $\mathrm{K}^{+}$channel activation causing vasodilation, via the common pathway of CGRP release from perivascular sensory nerve endings.

\subsubsection{Role of TRPA1 and TRPM8 channels in intrinsic vascular responses to cold}

Our results provide evidence that TRPA1 and TRPM 8 channels are essential vascular sensors of cold, playing a significant role in a local vascular response to cold temperatures in isolated arteries when the CNS is not mediating the response. Human studies have already described a cold systemic reflex at very low temperatures $\left(<15{ }^{\circ} \mathrm{C}\right)$; however, if these channels are present in peripheral nerves endings in the adventitia of the vessels, they could also modulate the cutaneous circulation in response to local temperature changes as a protective mechanism. Therefore, in this study, we used a cold exposure model to measure intrinsic responses after local cooling exposure of isolated plantar arteries from $\mathrm{KO}$ mice and their corresponding controls. Immediately after local cold exposure of plantar arteries from c57bl/6j mice ( $10^{\circ} \mathrm{C}$ for at least $\left.5 \mathrm{~min}\right)$, a decreased in the diameter was observed, that was fully reverted back to baseline levels when returning to the initial temperature $\left(37^{\circ} \mathrm{C}\right)$.

The local cold-induced vascular response found in this study did not involve the reported Hunting reaction, which induces vasodilation in response to cold stimulus and is characterized by subsequent vasoconstrictions and vasodilations during the cooling phase [176]. 
Deletion of both TRPV4 and TRPM3 channels, suggested as environmental thermosensors, did not affect the response to cold, consistent with the knowledge that these channels have been reported to play a role as heat sensors $[62,215]$. However ${ }_{2}$ the role of TRPA1 in cold sensation is still a big debate in literature. Several studies have shown an indirect activation of TRPA1 channel under low temperatures [180]. The first evidence of the role of TRPA1 in cold sensitivity was shown in $\mathrm{CHO}$ cells transfected with TRPA1 [91], but other groups were unable to reproduce similar data in HEK293 cells [77]. Also, while Munns et al. showed no correlation between TRPA1 and cold temperature in cultured DRG neurons [229], other groups have demonstrated that DRG neurons are cold sensitive via TRPA1 activation [92]. Several reasons have been proposed for the different findings between studies, such as different cell types and methodologies. In spite of some discrepancies, it seems that there is substantial evidence for a role of TRPA1 as a cold sensor. However, we do not know many details regarding the mechanisms and the functional contribution of this channel in physiological and pathophysiological situations. In particular, the knowledge and understanding of TRPA1 contribution to cold-induced vascular response is still at an early stage. Karashima et al. showed that cold-induced nociceptive behavior in mice is TRPA1-dependent [181]. TRPA1 has been proposed to play an essential role in the mechanisms involved in systemic vascular responses induced by cold [190]. In this line, we demonstrated that algo the intrinsic vascular response to cold is modulated by TRPA1 activation. The cold-induced vasoconstriction found in precontracted isolated plantar arteries, exposed to low temperatures $\left(10^{\circ} \mathrm{C}\right)$, was substantially reduced in Trpa1 KO plantar arteries and in c57bl/6j pretreated with the TRPA1 antagonist $\mathrm{HCO30031.}$

Another interesting point of debate is the comparison of the responses to cold of TRPA1 and TRPM8 channels. It has been suggested that both are cold sensors that could differ on the range of activating temperatures: while TRPA1 would be responsible for sensing a noxious cold range, TRPM8 is suggested to sense innocuous cool temperature [230]. In addition, it has been reported that TRPM8 expressed on sensory neurons mediates the response to deep body cooling which is associated with cutaneous vasoconstriction [191]. In this line, Reimundez et al. showed that Trpm8 $\mathrm{KO}$ mice exhibited a fall of $0.7^{\circ} \mathrm{C}$ in core body temperature when housed at cold temperatures and TRPM 8 deficiency induced an increase in tail heat loss demonstrating that TRPM8 is required for a precise thermoregulation in response to cold [231]. Although, the control of body temperature in the systemic response 
is thought to be the main factor regulating blood flow in the cutaneous circulation. We decided to investigate the contribution of TRPM8 channels to the local vascular response to cold. We found that in TRPM $8+/+$ mice, cold was able to produce a significant vasoconstriction in isolated plantar arteries, and this response was decreased in Trpm8 KO plantar arteries. This suggests that TRPM8 is also involved in the intrinsic cold-induced vascular response affecting the cutaneous circulation without the presence of the CNS. Our ex vivo approach does not allow us to explore the relative contribution of these channels (TRPA1 and TRPM8) to both intrinsic and systemic responses to cold, so that we cannot determine the effect of TRPA1 and TRPM8 channels in thermoregulatory responses. However, we provided evidence of a local role of these channels in cold-induced vascular responses. The fact that the outcome of this local response is also a vasoconstriction suggest that both local and global contributions of TRPA1 and TRPM8 to cold-induced vascular response are additive, so that local mechanisms can reinforce systemic reflexes, contributing in a more direct and fast way to vascular reactivity in response to temperature changes.

TRPM8 and TRPA1 channels have been proposed to be coexpressed in the same neurons. For instance, in cultured DRG, a population of cells has been reported to be sensitive to the TRPA1 and TRPM8 channels agonists mustard oil and menthol [232]. In another study, 20\% of TRPA1positive neurons also express TRPM8 [233]. Using Trpm8 KO plantar arteries in the presence of the TRPA1 antagonist (HC030031), we showed that the cold-induced vasoconstriction was completely abolished. Altogether, these results confirmed that local cold-induced vascular response is mediated via both cold thermo-TRP channels TRPA1 and TRPM 8 located in a subset of perivascular nerves, and that these channels work in a synergistic manner to mediate vasoconstriction. This additive effect could be explained by the activation of these channels in a different range of low temperatures, so that TRPM8 would be activated under mild cooling temperatures, while lower temperatures would recruit TRPA1 channels. In agreement with this, Winter et $a l$. [196] found that these channels are responsible to influence the behavioral responses over the entire cold temperature range. However, it is not clear whether TRPM8 is involved in both innocuous and/or noxious cold transduction. Bautista et al. showed that Trpm8 KO mice display no preference for warm surface under temperatures down to $15{ }^{\circ} \mathrm{C}$, suggesting that mice lacking TRPM 8 channels cannot discriminate between warm and innocuous cool temperature; however, when the temperature drop into a noxious range $\left(5-10^{\circ} \mathrm{C}\right), \operatorname{Trpm} 8 \mathrm{KO}$ mice display a preference for the warm side [150]. These findings 
were supported by Dhaka et al. suggesting not only a TRPM8-dependent mechanism for innocuous cold sensation, but also the presence of a TRPM8-independent mechanism for noxious cold transduction [152]. In accordance with this, Pan et al. have reported that activation of both channels by using a copper cold probe $\left(5^{\circ} \mathrm{C}\right)$ affect the blood flow under localized cold conditions; however, neither the TRPA1 antagonist nor the TRPM8 antagonist had effects in modulating the blood flow response induced by the localized cooling of the mouse paw. Intriguingly, other cold- sensing mechanisms have been suggested that are independent of TRPA1 and TRPM8 [229].

In contrast, we showed a prominent role of TRPA1 and TRPM8 expressed in sympathetic neurons in mediating the cold vascular response at a noxious cold range. However, whether TRPM8 channels can also be influenced in the innocuous cold range requires further investigation. The study in our experimental conditions of the effect of vessel diameter of a range of temperatures between 25 and $10{ }^{\circ} \mathrm{C}$, to determine the degree of activation of both TRPM8 and TRPA1 channels could help to check this hypothesis.

The relative contribution of sensory and sympathetic nerves to this local response to cold is difficult to determine. It is known that they have a similar distribution pattern around blood vessels in the adventitial layer and an influence between the two nerve subsets was shown after denervation of sensory peptide- or catecholamine-containing sympathetic nerves [234]. In this study, we pretreated c57bl/6j plantar arteries with BIBN, a well-known CGRP receptor antagonist used for the treatment of migraine [23] and we found a significantly increase of the vasoconstrictor response, implying the involvement of sensory perivascular nerves in the local vascular response to cold. On the other hand, superficial vessels exhibit $\alpha$ adrenoreceptors mediated peripheral vasoconstriction during exposures to low temperatures $[235,236]$. Using a pharmacological blockade of catecholamine release by guanethidine caused not only an inhibitory effect on the cold-induced vasoconstriction but also a significant vasodilation in response to cold.

Altogether, our data indicated the contribution of TRPM8 and TRPA1 channels located in both sensory and sympathetic nerve endings to this intrinsic cold-induced response.

While the expression of these channels in sensory nerve endings is well documented, the data in the literature regarding the presence of TRPA1 and TRPM8 in sympathetic nerves and their functional role is scarce and far from being conclusive. Smith et al. suggested that 
the sympathetic nervous system mediated by the activation of TRPA1 may play a direct role in sympathetic responses to cold temperatures. Using $\mathrm{Ca}^{2+}$ imaging, they have revealed a population of neurons in the SCG of the mouse that respond to cooling but were insensitive to menthol [237]. In another study from the same group, they found that cold sensitivity in sympathetic neurons and sensory neurons can be generated in the absence of TRPM 8 and TRPA1 after the followed application of agonists [229]. In our study, we were able to detect Trpa1 and Trpm8 mRNA expression in isolated SCG from c57bl/6j. In addition, we demonstrated that the local vasoconstrictor response is dominated by the activation by cold of TRPA1 and TRPM8 channels located in the sympathetic nerve endings. Finally, we showed that this cold-induced vascular response was fully abolished when blocking both TRPA1 and TRPM8 receptors. Altogether, these data suggest that sympathetic fibers play an essential role into the intrinsic cold-induced vascular reactivity and that TRPA1 and TRPM8 channels are fully mediating this vascular response.

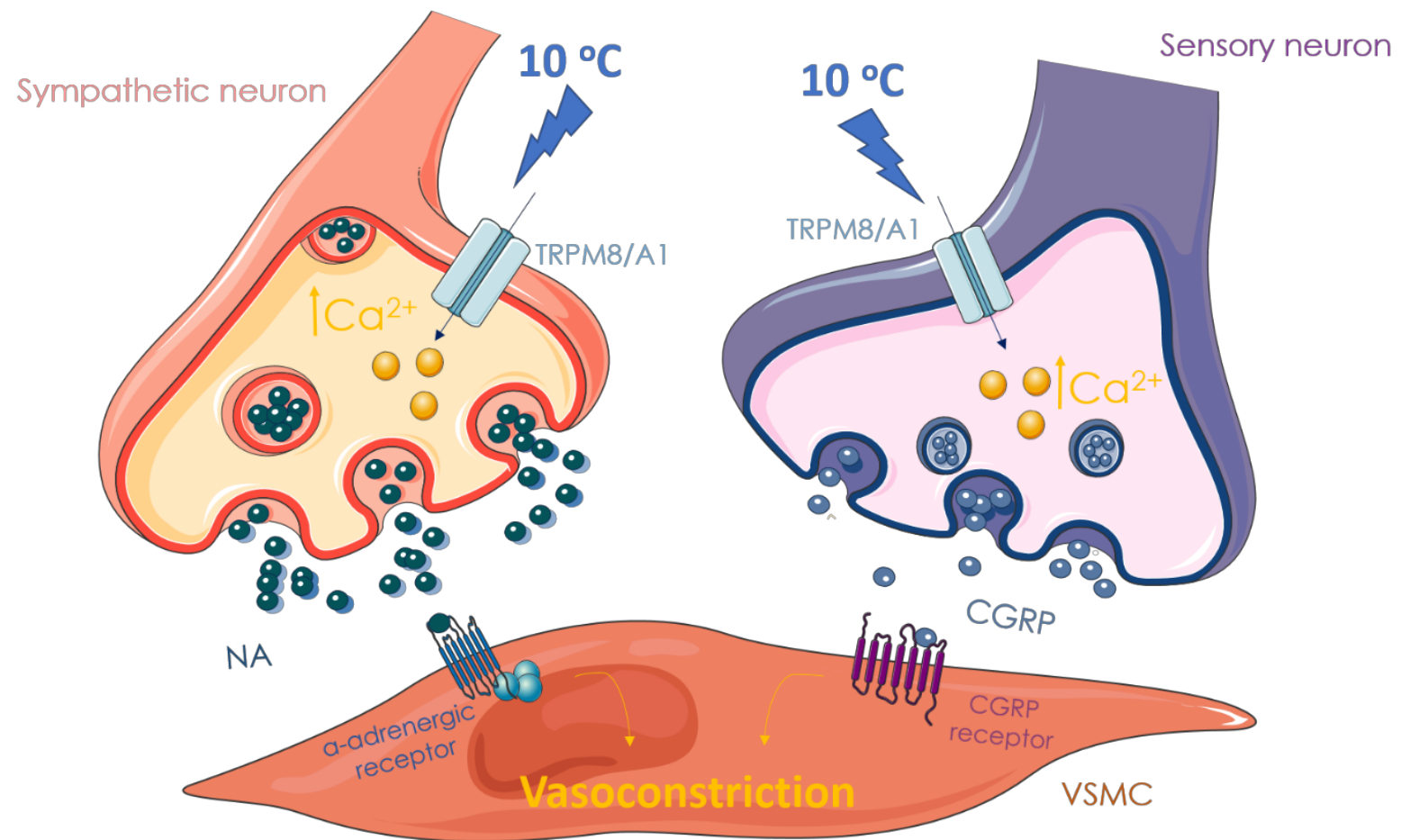

Figure 6.2. Proposed cold-induced vascular response pathway in plantar arteries. Activation of TRPA1 and TRPM8 channels by cold stimulus leads to vasoconstriction of plantar arteries via the release of neuropeptides by sensory and sympathetic nerves. 
Collectively, these novel evidences revealed an important role of TRPA1 and TRPM8 channels in mediating the vascular response to local cold exposure in isolated cutaneous arteries without the involvement of the CNS. Their activation by low temperatures is compatible with a dual mechanism in which TRPA1 and TRPM 8 channels are expressed in both sympathetic and sensory neurons and lead to antagonist effects through the release of neurotransmitters from both types of fibres and resulting in a global vasocontriction of the artery (Figure 6.2). Our results demonstrate the presence of TRPA1 and TRPM 8 channels not only in afferent (sensory) fibres but also in efferent (sympathetic) nerve fibres and provide the first direct evidence for an intrinsic local response to cold in isolated cutaneous arteries. Due to their location in sympathetic nerve endings and their relevant contribution to coldinduced vasoconstriction it is tempting to speculate that these channels may be relevant in the development of diseases such as Raynaud's disease in which low temperatures induces a robust vasoconstriction of the peripheral blood vessels via activation of sympathetic nerves leading to hypoxia tissue [238].

Since, avoiding sudden cold and rapid changes in temperature are the primary therapies recommended until now [239]. TRPA1 and TRPM8 channels could represent novel targets for the development of therapies directed to attenuate sympathetic vasoconstriction.

\subsection{General conclusions}

Although several sensory TRP channels have been implicated in the regulation of the vascular tone via multiple mechanisms, the role of the sensory TRPM3 channel in vascular function remains unknown. We found that, contrary to what was reported for aorta [128], PS induces vasodilation of mesenteric arteries, and that these effects are partly mediated by activation of TRPM3, via release of CGRP and subsequent activation of $\mathrm{K}^{+}$channels in VSMC. Our data support a contribution of TRPM3 as a potential therapeutic target for the modulation of the tone of resistance arteries and as a plausible effector of endogenous damage-associated molecules mediating protective responses in these vascular beds.

On the other hand, vascular functions of the sensory TRP channels have been also associated with their thermo-properties [179]. In this sense, we found that TRPA1 and TRPM8 channels have a key role in the local cold-induced vascular response. Using myography experiments to determine changes in vessel diameter, we could identify these channels both in sensory and sympathetic nerve endings, and we could conclude that the global cold-induced 
vasoconstriction observed in the plantar artery is the sum of a small vasodilatory response (due to activation of these channels in sensory nerve endings) and a large vasoconstrictor response (due to the activation of the channels present in sympathetic nerve endings). These results represent the first evidence for an intrinsic response to cold in cutaneous arteries and for the presence and the functional role of sensory TRP channels in efferent nerve fibers.

\subsection{Future perspectives}

\subsubsection{Role of TRPM3 in hypertension.}

Hypertension remains an important public health concern and a major-medical challenge, being one of the most important risk factor for heart disease and stroke and carrying significant mortality and morbidity. It has been found that this disease is associated to alterations in the function of the vasculature (myogenic response) [240, 241]. However, the mechanisms underlying blood pressure deregulation are not fully understood.

The contribution of TRPM3 to systemic blood pressure regulation is difficult to predict, since activation of TRPM3 was also reported to increase the tone of mouse aorta [128]. Preliminary data demonstrated that Trpm3 KO mice to be slightly but consistently hypotensive compared to $\mathrm{c57bl} / 6 \mathrm{j}$ mice (Figure 6.3), indicating that TRPM3 contributes to mean blood pressure regulation and that the overall contribution of TRPM3 channels in physiological conditions is to increase the vascular tone. Using a mouse model of Angll-induced hypertension we found that Angll infusion induced an overt hypertensive phenotype in c57bl/6j mice but very much surprisingly, not in Trpm3 KO mice (Figure 6.4).

Notably, TRPM3 is abundantly expressed in the kidneys [123], where are known to regulate the hypertensive effects of AngIl. Therefore, this suggest a role of this channel in mesenteric and renal arteries in the pathogenesis of hypertension. The future perspectives aim at understanding the role of TRPM 3 in hypertension.

To accomplish this, we propose to determine the expression profile of TRP and especially TRPM3 in renal arteries from c57bl/6j and Trpm3 KO mice. This should be done in mice in which hypertension will be induced via osmotic minipumps loaded with Angll (or saline for producing control animals for comparison). Real-time qPCR experiments and anatomical localization studies using immunofluorescence confocal microscopy could help to assess the levels of TRPM3 functional expression in renal arteries. 

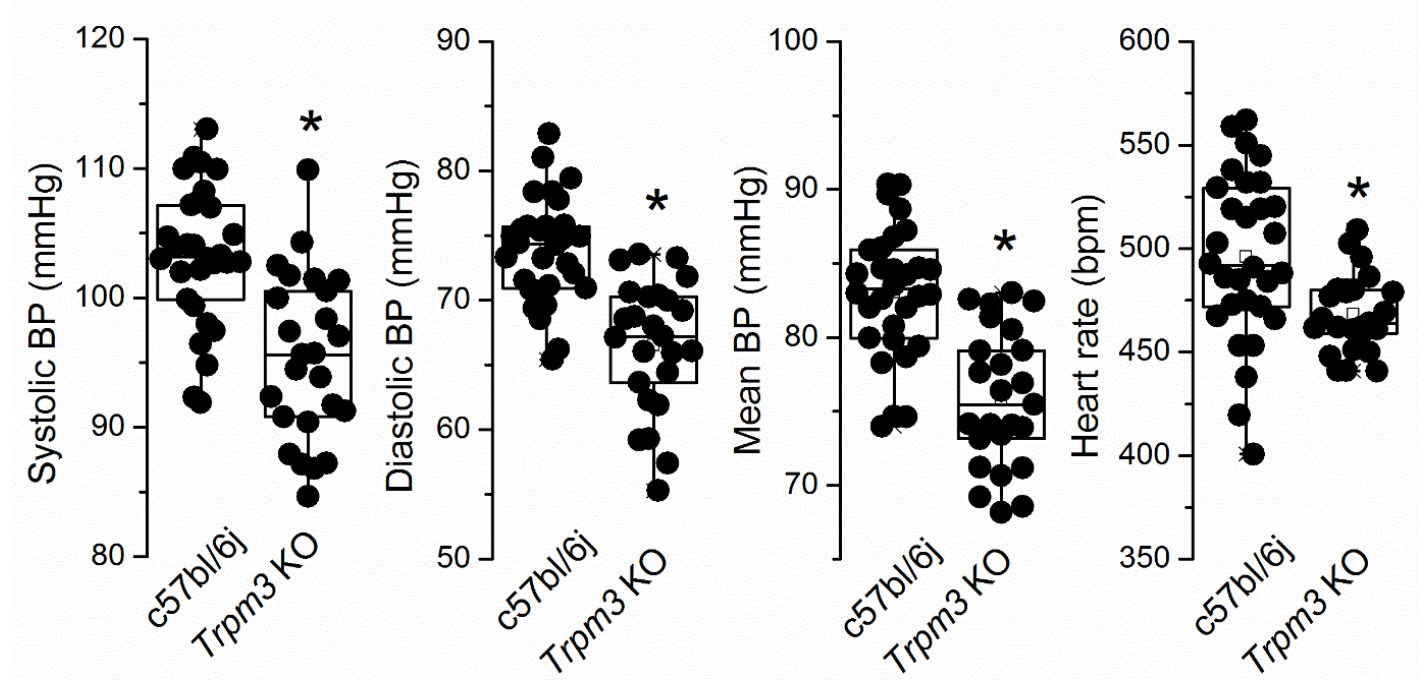

Figure 6.3. Box plot and individual systolic, diastolic, mean blood pressure and heart rate measurements in $\mathrm{c} 57 \mathrm{bl} / 6 \mathrm{j}$ mice $(\mathrm{n}=30)$ and Trpm3 KO mice $(n=25)$. Each data point is the average of value obtained in different sessions with 30 measurements per session. ${ }^{*}$ indicates $P<0.05$ for the comparison between groups.

Patch-clamp experiments in VSMC using TRPM3 agonists could serve to confirm the localization data at a functional level. In order to characterize the mechanism underlying in the effect of TRPM3-induced under hypertensive conditions by Angll, pressure myography experiments in renal arteries isolated from c57bl/6j and Trpm3 KO mice need to be performed in normal condition and after Angll-induced hypertension. This would help us to elucidate the mechanism underlying the key role of TRPM3 and test whether this channel serves as a novel therapeutic target for the treatment of high blood pressure.

Another important aspect is the expression of TRP channels in the context of remodelling of VSMC in Angll-induced hypertension. During the progression of hypertension VSMC undergo processes of structural remodelling, characterized by a transition from contractile to proliferative phenotypes [242]. Therefore, qPCR experiments in freshly isolated renal VSMC (contractile phenotype) and cultured renal VSMC (proliferative phenotype) would determine the TRP channel expression pattern in hypertensive and control mice giving us an idea whether other TRP channels are involved in the process of vascular remodelling induced by hypertension and therefore to establish new lines of research. 

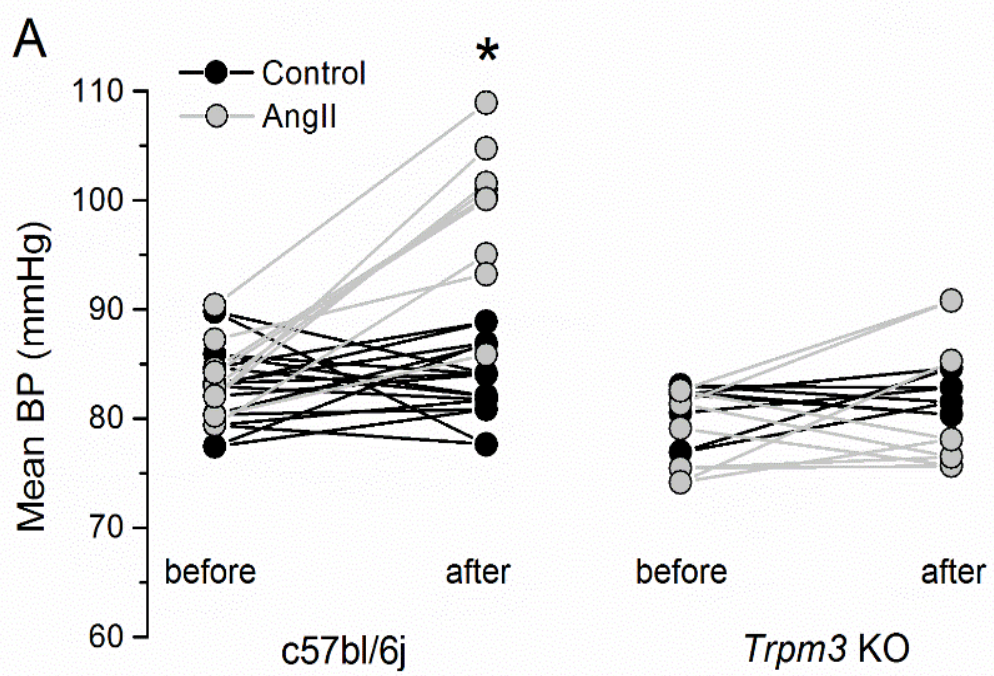

Figure 6.4. Individual mean blood pressure measurements before and after 7 days' treatment with control saline or angiotensin in c57bl/6j $(n=9)$ and Trpm3 KO $(n=5)$ mice. * indicates $P<0.05$ for the comparison of the data collected before and after treatment, paired t-test.

\subsubsection{Cold shock response in 'in vivo' models: role of TRPA1 and TRPM8 channels}

Cold shock response is the physiological response to sudden cold, especially cold water. It lasts for only 1 minute after entering the water. In humans, cold sock response is the most common cause of death from accidentally falling or voluntary immersion in very cold water. The first reactions after the immediate shock of cold are an automatic gasp reflex in response to rapid skin cooling and hyperventilation [243]. It also induces heart attack, especially in people with existing cardiovascular diseases, due to the heart has to pump the same volume of blood through the body. Cold shock response has been associated with a robust vasoconstriction of the peripheral arteries [244], however, the molecular targets and the mechanisms underlying this cold-induced systemic response are not fully understood.

TRPM8 has been identified as an essential receptor for initiating the cold shock response in testes [245]. These results together with our preliminary data showing a role of TRPA1 and TRPM8 in response to cold in isolated peripheral arteries suggest that these sensory TRP channels could be also involved in the vascular cold shock response. Therefore, the second aim of the future perspectives would be to investigate the influence of TRPA1 and TRPM 8 channels in the hemodynamic responses to fast cold application in 'in vivo' models.

First, we propose to challenge Trpa1 KO, Trpm8 KO mice and their corresponding controls to noxious cold water $\left(10^{\circ} \mathrm{C}\right)$, by immersion during 30 seconds while heart rate and arterial 
pressure are measured by telemetry system in conscious mice. This system requires a surgical implantation of a catheter into the left carotid artery of the mouse which is attached to a combination pressure transducer, transmitter and battery, all encapsulated in an implantable microminiaturized electronic monitor which is placed subcutaneously in the mouse. We would also use different pharmacological tools including channel antagonists and blockers of both sympathetic and sensory nerves that could serve to confirm the implication of both nervous systems.

Next, we could also determine whether noxious cold stimulus 'in vivo' activates TRPA1 and TRPM8 channels by using the non-invasively, computerized tail-cuff system. Measurements of systolic and diastolic pressure and heart rate of trpa1 $\mathrm{KO}$, trpm8 $\mathrm{KO}$ mice and their corresponding controls would be acquired while awake animals are placed in plastic restrainers. A cuff with a pneumatic pulse sensor would be attached to the tail. First, they will be kept warm in a platform heated to $32-35^{\circ} \mathrm{C}$ for 5 minutes and afterwards on frozen blocks during 1 minute. To obtain appropriate blood pressure and heart rate estimations, the measurements would be carried out in at least three different days, and 20-40 consecutive measurements would be obtained in each session from each animal. We would use pharmacological approaches to elucidate the mechanism underlying the cold response.

Altogether, these future perspectives will give us some ideas about the mechanism underlying the fast-cold application response and whether TRPA1 and TRPM8 channels are involved in the hemodynamic responses 'in vivo' induced by noxious cold application. 
References 
[1] J.R. Levick, An Introduction to Cardiovascular Physiology, (2003).

[2] M.T. Nelson, J.M. Quayle, Physiological roles and properties of potassium channels in arterial smooth muscle, Am J Physiol 268(4 Pt 1) (1995) C799-822.

[3] W.C. Aird, Phenotypic heterogeneity of the endothelium: II. Representative vascular beds, Circ Res 100(2) (2007) 174-90.

[4] J.C. McGrath, C. Deighan, A.M. Briones, M.M. Shafaroudi, M. McBride, J. Adler, S.M. Arribas, E. Vila, C.J. Daly, New aspects of vascular remodelling: the involvement of all vascular cell types, Exp Physiol 90(4) (2005) 469-75.

[5] J.R. Levick, C.C. Michel, Microvascular fluid exchange and the revised Starling principle, Cardiovasc Res 87(2) (2010) 198-210.

[6] E.B. Westcott, S.S. Segal, Perivascular innervation: a multiplicity of roles in vasomotor control and myoendothelial signaling, Microcirculation 20(3) (2013) 217-38.

[7] L.J. Heller, D.E. Mohrman, J.A. Smith, K.B. Wallace, Multitrack system for superfusing isolated cardiac myocytes, Am J Physiol Heart Circ Physiol 284(5) (2003) H1872-8.

[8] Y. Sheng, L. Zhu, The crosstalk between autonomic nervous system and blood vessels, Int J Physiol Pathophysiol Pharmacol 10(1) (2018) 17-28.

[9] G.E. Torres, R.R. Gainetdinov, M.G. Caron, Plasma membrane monoamine transporters: structure, regulation and function, Nat Rev Neurosci 4(1) (2003) 13-25.

[10] V. Lo Martire, A. Silvani, S. Alvente, S. Bastianini, C. Berteotti, A. Valli, G. Zoccoli, Modulation of sympathetic vasoconstriction is critical for the effects of sleep on arterial pressure in mice, J Physiol (2017).

[11] Y. Tanaka, T. Horinouchi, K. Koike, New insights into beta-adrenoceptors in smooth muscle: distribution of receptor subtypes and molecular mechanisms triggering muscle relaxation, Clin Exp Pharmacol Physiol 32(7) (2005) 503-14.

[12] J.P. Cooke, J.M. Marshall, Mechanisms of Raynaud's disease, Vasc Med 10(4) (2005) 293307.

[13] D.S. Goldstein, Arterial baroreflex sensitivity, plasma catecholamines, and pressor responsiveness in essential hypertension, Circulation 68(2) (1983) 234-40.

[14] L. Landsberg, Insulin sensitivity in the pathogenesis of hypertension and hypertensive complications, Clin Exp Hypertens 18(3-4) (1996) 337-46.

[15] S. Sorota, The sympathetic nervous system as a target for the treatment of hypertension and cardiometabolic diseases, J Cardiovasc Pharmacol 63(5) (2014) 466-76.

[16] F. Mahfoud, M. Schlaich, I. Kindermann, C. Ukena, B. Cremers, M.C. Brandt, U.C. Hoppe, O. Vonend, L.C. Rump, P.A. Sobotka, H. Krum, M. Esler, M. Bohm, Effect of renal sympathetic denervation on glucose metabolism in patients with resistant hypertension: a pilot study, Circulation 123(18) (2011) 1940-6.

[17] D. Francis, J. Diorio, D. Liu, M.J. Meaney, Nongenomic transmission across generations of maternal behavior and stress responses in the rat, Science 286(5442) (1999) 1155-8.

[18] Y.S. Mukouyama, D. Shin, S. Britsch, M. Taniguchi, D.J. Anderson, Sensory nerves determine the pattern of arterial differentiation and blood vessel branching in the skin, Cell 109(6) (2002) 693-705.

[19] S.D. Brain, A.D. Grant, Vascular actions of calcitonin gene-related peptide and adrenomedullin, Physiol Rev 84(3) (2004) 903-34.

[20] F. Van Valen, G. Piechot, H. Jurgens, Calcitonin gene-related peptide (CGRP) receptors are linked to cyclic adenosine monophosphate production in SK-N-MC human neuroblastoma cells, Neurosci Lett 119(2) (1990) 195-8. 
[21] J. Li, C.V. Vause, P.L. Durham, Calcitonin gene-related peptide stimulation of nitric oxide synthesis and release from trigeminal ganglion glial cells, Brain Res 1196 (2008) 22-32.

[22] F.A. Russell, R. King, S.J. Smillie, X. Kodji, S.D. Brain, Calcitonin gene-related peptide: physiology and pathophysiology, Physiol Rev 94(4) (2014) 1099-142.

[23] J. Olesen, H.C. Diener, I.W. Husstedt, P.J. Goadsby, D. Hall, U. Meier, S. Pollentier, L.M. Lesko, B.B.C.P.o.C.S. Group, Calcitonin gene-related peptide receptor antagonist BIBN 4096 BS for the acute treatment of migraine, N Engl J Med 350(11) (2004) 1104-10.

[24] A.P. Ho, C.G. Dahlof, S.D. Silberstein, J.R. Saper, M. Ashina, J.T. Kost, S. Froman, H. Leibensperger, C.R. Lines, T.W. Ho, Randomized, controlled trial of telcagepant over four migraine attacks, Cephalalgia 30(12) (2010) 1443-57.

[25] R. Huang, A. Karve, I. Shah, M.C. Bowers, D.J. DiPette, S.C. Supowit, G.S. Abela, Deletion of the mouse alpha-calcitonin gene-related peptide gene increases the vulnerability of the heart to ischemia-reperfusion injury, Am J Physiol Heart Circ Physiol 294(3) (2008) H1291-7.

[26] Y. Li, R.R. Fiscus, J. Wu, L. Yang, X. Wang, The antiproliferative effects of calcitonin generelated peptide in different passages of cultured vascular smooth muscle cells, Neuropeptides 31(5) (1997) 503-9.

[27] W. Wang, W. Sun, X. Wang, Intramuscular gene transfer of CGRP inhibits neointimal hyperplasia after balloon injury in the rat abdominal aorta, Am J Physiol Heart Circ Physiol 287(4) (2004) H1582-9.

[28] X.P. Qin, F. Ye, C.P. Hu, D.F. Liao, H.W. Deng, Y.J. Li, Effect of calcitonin gene-related peptide on angiotensin II-induced proliferation of rat vascular smooth muscle cells, Eur J Pharmacol 488(1-3) (2004) 45-9.

[29] Z. Zhou, C.P. Hu, C.J. Wang, T.T. Li, J. Peng, Y.J. Li, Calcitonin gene-related peptide inhibits angiotensin II-induced endothelial progenitor cells senescence through up-regulation of klotho expression, Atherosclerosis 213(1) (2010) 92-101.

[30] L. Edvinsson, Calcitonin gene-related peptide (CGRP) and the pathophysiology of headache: therapeutic implications, CNS Drugs 15(10) (2001) 745-53.

[31] P.J. Goadsby, Neurovascular headache and a midbrain vascular malformation: evidence for a role of the brainstem in chronic migraine, Cephalalgia 22(2) (2002) 107-11.

[32] L. Edvinsson, R. Alm, D. Shaw, R.Z. Rutledge, K.S. Koblan, J. Longmore, S.A. Kane, Effect of the CGRP receptor antagonist BIBN4096BS in human cerebral, coronary and omental arteries and in SK-N-MC cells, Eur J Pharmacol 434(1-2) (2002) 49-53.

[33] U. Arulmani, M.P. Schuijt, J.P. Heiligers, E.W. Willems, C.M. Villalon, P.R. Saxena, Effects of the calcitonin gene-related peptide (CGRP) receptor antagonist BIBN4096BS on alphaCGRP-induced regional haemodynamic changes in anaesthetised rats, Basic Clin Pharmacol Toxicol 94(6) (2004) 291-7.

[34] S.J. Smillie, R. King, X. Kodji, E. Outzen, G. Pozsgai, E. Fernandes, N. Marshall, P. de Winter, R.J. Heads, C. Dessapt-Baradez, L. Gnudi, A. Sams, A.M. Shah, R.C. Siow, S.D. Brain, An ongoing role of alpha-calcitonin gene-related peptide as part of a protective network against hypertension, vascular hypertrophy, and oxidative stress, Hypertension 63(5) (2014) 1056-62. [35] W.F. Jackson, lon channels and vascular tone, Hypertension 35(1 Pt 2) (2000) 173-8.

[36] L. Alonso-Carbajo, M. Kecskes, G. Jacobs, A. Pironet, N. Syam, K. Talavera, R. Vennekens, Muscling in on TRP channels in vascular smooth muscle cells and cardiomyocytes, Cell Calcium 66 (2017) 48-61.

[37] Y. Pan, D. Thapa, L. Baldissera, Jr., F. Argunhan, A.A. Aubdool, S.D. Brain, Relevance of TRPA1 and TRPM8 channels as vascular sensors of cold in the cutaneous microvasculature, Pflugers Arch (2017). 
[38] Y. Takahashi, H. Watanabe, M. Murakami, T. Ohba, M. Radovanovic, K. Ono, T. Iijima, H. Ito, Involvement of transient receptor potential canonical 1 (TRPC1) in angiotensin II-induced vascular smooth muscle cell hypertrophy, Atherosclerosis 195(2) (2007) 287-96.

[39] S.I. Yeon, J.Y. Kim, D.S. Yeon, J. Abramowitz, L. Birnbaumer, S. Muallem, Y.H. Lee, Transient receptor potential canonical type 3 channels control the vascular contractility of mouse mesenteric arteries, PLoS One 9(10) (2014) e110413.

[40] R.A. Fernandez, J. Wan, S. Song, K.A. Smith, Y. Gu, M. Tauseef, H. Tang, A. Makino, D. Mehta, J.X. Yuan, Upregulated expression of STIM2, TRPC6, and Orai2 contributes to the transition of pulmonary arterial smooth muscle cells from a contractile to proliferative phenotype, Am J Physiol Cell Physiol 308(8) (2015) C581-93.

[41] A. Di, A.B. Malik, TRP channels and the control of vascular function, Curr Opin Pharmacol 10(2) (2010) 127-32.

[42] C. Guibert, T. Ducret, J.P. Savineau, Voltage-independent calcium influx in smooth muscle, Prog Biophys Mol Biol 98(1) (2008) 10-23.

[43] G.C. Amberg, M.F. Navedo, Calcium dynamics in vascular smooth muscle, Microcirculation 20(4) (2013) 281-9.

[44] S. Earley, J.E. Brayden, Transient receptor potential channels and vascular function, Clin Sci (Lond) 119(1) (2010) 19-36.

[45] B. Nilius, T. Voets, TRP channels: a TR(I)P through a world of multifunctional cation channels, Pflugers Arch 451(1) (2005) 1-10.

[46] C. Montell, G.M. Rubin, Molecular characterization of the Drosophila trp locus: a putative integral membrane protein required for phototransduction, Neuron 2(4) (1989) 1313-23.

[47] B. Nilius, F. Mahieu, Y. Karashima, T. Voets, Regulation of TRP channels: a voltage-lipid connection, Biochem Soc Trans 35(Pt 1) (2007) 105-8.

[48] D.E. Clapham, D. Julius, C. Montell, G. Schultz, International Union of Pharmacology. XLIX. Nomenclature and structure-function relationships of transient receptor potential channels, Pharmacol Rev 57(4) (2005) 427-50.

[49] B. Nilius, A. Szallasi, Transient receptor potential channels as drug targets: from the science of basic research to the art of medicine, Pharmacol Rev 66(3) (2014) 676-814.

[50] M. Liao, E. Cao, D. Julius, Y. Cheng, Structure of the TRPV1 ion channel determined by electron cryo-microscopy, Nature 504(7478) (2013) 107-12.

[51] E. Cao, M. Liao, Y. Cheng, D. Julius, TRPV1 structures in distinct conformations reveal activation mechanisms, Nature 504(7478) (2013) 113-8.

[52] C.E. Paulsen, J.P. Armache, Y. Gao, Y. Cheng, D. Julius, Structure of the TRPA1 ion channel suggests regulatory mechanisms, Nature 525(7570) (2015) 552.

[53] Y. Yin, M. Wu, L. Zubcevic, W.F. Borschel, G.C. Lander, S.Y. Lee, Structure of the cold- and menthol-sensing ion channel TRPM8, Science 359(6372) (2018) 237-241.

[54] K. Saotome, A.K. Singh, M.V. Yelshanskaya, A.I. Sobolevsky, Crystal structure of the epithelial calcium channel TRPV6, Nature 534(7608) (2016) 506-11.

[55] A. Dietrich, V. Chubanov, H. Kalwa, B.R. Rost, T. Gudermann, Cation channels of the transient receptor potential superfamily: their role in physiological and pathophysiological processes of smooth muscle cells, Pharmacol Ther 112(3) (2006) 744-60.

[56] C. Montell, TRP channels in Drosophila photoreceptor cells, J Physiol 567(Pt 1) (2005) 4551.

[57] L.J. Wu, T.B. Sweet, D.E. Clapham, International Union of Basic and Clinical Pharmacology. LXXVI. Current progress in the mammalian TRP ion channel family, Pharmacol Rev 62(3) (2010) 381-404. 
[58] B. Nilius, Transient receptor potential (TRP) cation channels: rewarding unique proteins, Bull Mem Acad R Med Belg 162(3-4) (2007) 244-53.

[59] B. Nilius, G. Owsianik, The transient receptor potential family of ion channels, Genome Biol 12(3) (2011) 218.

[60] M. Gees, B. Colsoul, B. Nilius, The role of transient receptor potential cation channels in Ca2+ signaling, Cold Spring Harb Perspect Biol 2(10) (2010) a003962.

[61] M.M. Moran, M.A. McAlexander, T. Biro, A. Szallasi, Transient receptor potential channels as therapeutic targets, Nat Rev Drug Discov 10(8) (2011) 601-20.

[62] J. Vriens, G. Owsianik, T. Hofmann, S.E. Philipp, J. Stab, X. Chen, M. Benoit, F. Xue, A. Janssens, S. Kerselaers, J. Oberwinkler, R. Vennekens, T. Gudermann, B. Nilius, T. Voets, TRPM3 is a nociceptor channel involved in the detection of noxious heat, Neuron 70(3) (2011) 482-94.

[63] J. Vriens, T. Voets, A cellular pathway controlling functional plasma membrane incorporation of the cold sensor TRPM8, Temperature (Austin) 3(4) (2016) 521-523.

[64] V. Chubanov, S. Kubanek, S. Fiedler, L. Mittermeier, T. Gudermann, A. Dietrich, Renal Functions of TRP Channels in Health and Disease, in: nd, T.L.R. Emir (Eds.), Neurobiology of TRP Channels, Boca Raton (FL), 2017, pp. 187-212.

[65] H. Wallace, Airway Pathogenesis Is Linked to TRP Channels, in: nd, T.L.R. Emir (Eds.), Neurobiology of TRP Channels, Boca Raton (FL), 2017, pp. 251-264.

[66] K. Philippaert, R. Vennekens, The Role of TRP Channels in the Pancreatic Beta-Cell, in: nd, T.L.R. Emir (Eds.), Neurobiology of TRP Channels, Boca Raton (FL), 2017, pp. 229-250.

[67] A.D. Mickle, A.J. Shepherd, D.P. Mohapatra, Sensory TRP channels: the key transducers of nociception and pain, Prog Mol Biol Transl Sci 131 (2015) 73-118.

[68] K.J. Zappia, C.L. O'Hara, F. Moehring, K.Y. Kwan, C.L. Stucky, Sensory Neuron-Specific Deletion of TRPA1 Results in Mechanical Cutaneous Sensory Deficits, eNeuro 4(1) (2017).

[69] J.E. Brayden, S. Earley, M.T. Nelson, S. Reading, Transient receptor potential (TRP) channels, vascular tone and autoregulation of cerebral blood flow, Clin Exp Pharmacol Physiol 35(9) (2008) 1116-20.

[70] C. Moore, R. Gupta, S.E. Jordt, Y. Chen, W.B. Liedtke, Regulation of Pain and Itch by TRP Channels, Neurosci Bull (2017).

[71] M. Naziroglu, N. Braidy, Thermo-Sensitive TRP Channels: Novel Targets for Treating Chemotherapy-Induced Peripheral Pain, Front Physiol 8 (2017) 1040.

[72] R.H. Straub, TRPV1, TRPA1, and TRPM 8 channels in inflammation, energy redirection, and water retention: role in chronic inflammatory diseases with an evolutionary perspective, J Mol Med (Berl) 92(9) (2014) 925-37.

[73] O. Gouin, K. L'Herondelle, N. Lebonvallet, C. Le Gall-lanotto, M. Sakka, V. Buhe, E. PleeGautier, J.L. Carre, L. Lefeuvre, L. Misery, R. Le Garrec, TRPV1 and TRPA1 in cutaneous neurogenic and chronic inflammation: pro-inflammatory response induced by their activation and their sensitization, Protein Cell 8(9) (2017) 644-661.

[74] L. Vay, C. Gu, P.A. McNaughton, The thermo-TRP ion channel family: properties and therapeutic implications, Br J Pharmacol 165(4) (2012) 787-801.

[75] C.H. Tan, P.A. McNaughton, The TRPM2 ion channel is required for sensitivity to warmth, Nature 536(7617) (2016) 460-3.

[76] M.J. Caterina, A. Leffler, A.B. Malmberg, W.J. Martin, J. Trafton, K.R. Petersen-Zeitz, M. Koltzenburg, A.I. Basbaum, D. Julius, Impaired nociception and pain sensation in mice lacking the capsaicin receptor, Science 288(5464) (2000) 306-13. 
[77] S.E. Jordt, D.M. Bautista, H.H. Chuang, D.D. McKemy, P.M. Zygmunt, E.D. Hogestatt, I.D. Meng, D. Julius, Mustard oils and cannabinoids excite sensory nerve fibres through the TRP channel ANKTM1, Nature 427(6971) (2004) 260-5.

[78] D.M. Bautista, S.E. Jordt, T. Nikai, P.R. Tsuruda, A.J. Read, J. Poblete, E.N. Yamoah, A.I. Basbaum, D. Julius, TRPA1 mediates the inflammatory actions of environmental irritants and proalgesic agents, Cell 124(6) (2006) 1269-82.

[79] B.Y. Liu, Y. Tai, A.I. Caceres, S. Achanta, S. Balakrishna, X.M. Shao, J.F. Fang, S.E. Jordt, Oxidized Phospholipid OxPAPC Activates TRPA1 and Contributes to Chronic Inflammatory Pain in Mice, Plos One 11(11) (2016).

[80] N. Imamachi, G.H. Park, H. Lee, D.J. Anderson, M.I. Simon, A.I. Basbaum, S.K. Han, TRPV1expressing primary afferents generate behavioral responses to pruritogens via multiple mechanisms, Proc Natl Acad Sci U S A 106(27) (2009) 11330-5.

[81] D.F. Silva, M.M. de Almeida, C.G. Chaves, A.L. Braz, M.A. Gomes, L. Pinho-da-Silva, J.L. Pesquero, V.A. Andrade, F. Leite Mde, J.G. de Albuquerque, I.G. Araujo, X.P. Nunes, J.M. Barbosa-Filho, S. Cruz Jdos, A. Correia Nde, I.A. de Medeiros, TRPM8 Channel Activation Induced by Monoterpenoid Rotundifolone Underlies Mesenteric Artery Relaxation, PLoS One 10(11) (2015) e0143171.

[82] R. Vennekens, Emerging concepts for the role of TRP channels in the cardiovascular system, J Physiol 589(Pt 7) (2011) 1527-34.

[83] D. Jaquemar, T. Schenker, B. Trueb, An ankyrin-like protein with transmembrane domains is specifically lost after oncogenic transformation of human fibroblasts, J Biol Chem 274(11) (1999) 7325-33.

[84] Y. Karashima, J. Prenen, K. Talavera, A. Janssens, T. Voets, B. Nilius, Agonist-induced changes in $\mathrm{Ca}(2+)$ permeation through the nociceptor cation channel TRPA1, Biophys J 98(5) (2010) 773-83.

[85] J.A. Matta, P.M. Cornett, R.L. Miyares, K. Abe, N. Sahibzada, G.P. Ahern, General anesthetics activate a nociceptive ion channel to enhance pain and inflammation, Proc Natl Acad Sci U S A 105(25) (2008) 8784-9.

[86] K. Talavera, M. Gees, Y. Karashima, V.M. Meseguer, J.A. Vanoirbeek, N. Damann, W. Everaerts, M. Benoit, A. Janssens, R. Vennekens, F. Viana, B. Nemery, B. Nilius, T. Voets, Nicotine activates the chemosensory cation channel TRPA1, Nat Neurosci 12(10) (2009) 12939.

[87] M. Bandell, G.M. Story, S.W. Hwang, V. Viswanath, S.R. Eid, M.J. Petrus, T.J. Earley, A. Patapoutian, Noxious cold ion channel TRPA1 is activated by pungent compounds and bradykinin, Neuron 41(6) (2004) 849-57.

[88] N. Takahashi, Y. Mizuno, D. Kozai, S. Yamamoto, S. Kiyonaka, T. Shibata, K. Uchida, Y. Mori, Molecular characterization of TRPA1 channel activation by cysteine-reactive inflammatory mediators, Channels (Austin) 2(4) (2008) 287-98.

[89] D.A. Andersson, C. Gentry, S. Moss, S. Bevan, Transient receptor potential A1 is a sensory receptor for multiple products of oxidative stress, J Neurosci 28(10) (2008) 2485-94.

[90] Y. Sawada, H. Hosokawa, K. Matsumura, S. Kobayashi, Activation of transient receptor potential ankyrin 1 by hydrogen peroxide, Eur J Neurosci 27(5) (2008) 1131-42.

[91] G.M. Story, A.M. Peier, A.J. Reeve, S.R. Eid, J. Mosbacher, T.R. Hricik, T.J. Earley, A.C. Hergarden, D.A. Andersson, S.W. Hwang, P. Mclntyre, T. Jegla, S. Bevan, A. Patapoutian, ANKTM1, a TRP-like channel expressed in nociceptive neurons, is activated by cold temperatures, Cell 112(6) (2003) 819-29. 
[92] Y. Sawada, H. Hosokawa, A. Hori, K. Matsumura, S. Kobayashi, Cold sensitivity of recombinant TRPA1 channels, Brain Res 1160 (2007) 39-46.

[93] O. Fajardo, V. Meseguer, C. Belmonte, F. Viana, TRPA1 channels mediate cold temperature sensing in mammalian vagal sensory neurons: pharmacological and genetic evidence, J Neurosci 28(31) (2008) 7863-75.

[94] S. Zurborg, B. Yurgionas, J.A. Jira, O. Caspani, P.A. Heppenstall, Direct activation of the ion channel TRPA1 by Ca2+, Nat Neurosci 10(3) (2007) 277-9.

[95] J. Chen, D. Kang, J. Xu, M. Lake, J.O. Hogan, C. Sun, K. Walter, B. Yao, D. Kim, Species differences and molecular determinant of TRPA1 cold sensitivity, Nat Commun 4 (2013) 2501. [96] (!!! INVALID CITATION !!! [96]).

[97] Y. Jang, Y. Lee, S.M. Kim, Y.D. Yang, J. Jung, U. Oh, Quantitative analysis of TRP channel genes in mouse organs, Arch Pharm Res 35(10) (2012) 1823-30.

[98] C. Kunert-Keil, F. Bisping, J. Kruger, H. Brinkmeier, Tissue-specific expression of TRP channel genes in the mouse and its variation in three different mouse strains, BMC Genomics 7 (2006) 159.

[99] S. Earley, A.L. Gonzales, R. Crnich, Endothelium-dependent cerebral artery dilation mediated by TRPA1 and Ca2+-Activated K+ channels, Circ Res 104(8) (2009) 987-94.

[100] S. Earley, TRPA1 channels in the vasculature, Br J Pharmacol 167(1) (2012) 13-22.

[101] M.N. Sullivan, A.L. Gonzales, P.W. Pires, A. Bruhl, M.D. Leo, W. Li, A. Oulidi, F.A. Boop, Y. Feng, J.H. Jaggar, D.G. Welsh, S. Earley, Localized TRPA1 channel Ca2+ signals stimulated by reactive oxygen species promote cerebral artery dilation, Sci Signal 8(358) (2015) ra2.

[102] D.M. Bautista, P. Movahed, A. Hinman, H.E. Axelsson, O. Sterner, E.D. Hogestatt, D. Julius, S.E. Jordt, P.M. Zygmunt, Pungent products from garlic activate the sensory ion channel TRPA1, Proc Natl Acad Sci U S A 102(34) (2005) 12248-52.

[103] E.Y. Cheah, P.C. Burcham, T.S. Mann, P.J. Henry, Acrolein relaxes mouse isolated tracheal smooth muscle via a TRPA1-dependent mechanism, Biochem Pharmacol 89(1) (2014) 148-56. [104] A. Yanaga, H. Goto, T. Nakagawa, H. Hikiami, N. Shibahara, Y. Shimada, Cinnamaldehyde induces endothelium-dependent and -independent vasorelaxant action on isolated rat aorta, Biol Pharm Bull 29(12) (2006) 2415-8.

[105] J. Alvarez-Collazo, L. Alonso-Carbajo, A.I. Lopez-Medina, Y.A. Alpizar, S. Tajada, B. Nilius, T. Voets, J.R. Lopez-Lopez, K. Talavera, M.T. Perez-Garcia, J.L. Alvarez, Cinnamaldehyde inhibits L-type calcium channels in mouse ventricular cardiomyocytes and vascular smooth muscle cells, Pflugers Arch 466(11) (2014) 2089-99.

[106] M. Tominaga, M.J. Caterina, A.B. Malmberg, T.A. Rosen, H. Gilbert, K. Skinner, B.E. Raumann, A.I. Basbaum, D. Julius, The cloned capsaicin receptor integrates multiple painproducing stimuli, Neuron 21(3) (1998) 531-43.

[107] M.J. Caterina, M.A. Schumacher, M. Tominaga, T.A. Rosen, J.D. Levine, D. Julius, The capsaicin receptor: a heat-activated ion channel in the pain pathway, Nature 389(6653) (1997) 816-24.

[108] S.E. Jordt, M. Tominaga, D. Julius, Acid potentiation of the capsaicin receptor determined by a key extracellular site, Proc Natl Acad Sci U S A 97(14) (2000) 8134-9.

[109] T. Voets, G. Droogmans, U. Wissenbach, A. Janssens, V. Flockerzi, B. Nilius, The principle of temperature-dependent gating in cold- and heat-sensitive TRP channels, Nature 430(7001) (2004) 748-54.

[110] Y. Kajihara, M. Murakami, T. Imagawa, K. Otsuguro, S. Ito, T. Ohta, Histamine potentiates acid-induced responses mediating transient receptor potential V1 in mouse primary sensory neurons, Neuroscience 166(1) (2010) 292-304. 
[111] J.B. Davis, J. Gray, M.J. Gunthorpe, J.P. Hatcher, P.T. Davey, P. Overend, M.H. Harries, J. Latcham, C. Clapham, K. Atkinson, S.A. Hughes, K. Rance, E. Grau, A.J. Harper, P.L. Pugh, D.C. Rogers, S. Bingham, A. Randall, S.A. Sheardown, Vanilloid receptor-1 is essential for inflammatory thermal hyperalgesia, Nature 405(6783) (2000) 183-7.

[112] A. Toth, A. Czikora, E.T. Pasztor, B. Dienes, P. Bai, L. Csernoch, I. Rutkai, V. Csato, I.S. Manyine, R. Porszasz, I. Edes, Z. Papp, J. Boczan, Vanilloid receptor-1 (TRPV1) expression and function in the vasculature of the rat, J Histochem Cytochem 62(2) (2014) 129-44.

[113] T. Kark, Z. Bagi, E. Lizanecz, E.T. Pasztor, N. Erdei, A. Czikora, Z. Papp, I. Edes, R. Porszasz, A. Toth, Tissue-specific regulation of microvascular diameter: opposite functional roles of neuronal and smooth muscle located vanilloid receptor-1, Mol Pharmacol 73(5) (2008) 140512.

[114] D.J. Cavanaugh, A.T. Chesler, A.C. Jackson, Y.M. Sigal, H. Yamanaka, R. Grant, D. O'Donnell, R.A. Nicoll, N.M. Shah, D. Julius, A.I. Basbaum, Trpv1 reporter mice reveal highly restricted brain distribution and functional expression in arteriolar smooth muscle cells, J Neurosci 31(13) (2011) 5067-77.

[115] I.N. Bratz, G.M. Dick, J.D. Tune, J.M. Edwards, Z.P. Neeb, U.D. Dincer, M. Sturek, Impaired capsaicin-induced relaxation of coronary arteries in a porcine model of the metabolic syndrome, Am J Physiol Heart Circ Physiol 294(6) (2008) H2489-96.

[116] G. Guarini, V.A. Ohanyan, J.G. Kmetz, D.J. DelloStritto, R.J. Thoppil, C.K. Thodeti, J.G. Meszaros, D.S. Damron, I.N. Bratz, Disruption of TRPV1-mediated coupling of coronary blood flow to cardiac metabolism in diabetic mice: role of nitric oxide and BK channels, Am J Physiol Heart Circ Physiol 303(2) (2012) H216-23.

[117] J. Sun, Y. Pu, P. Wang, S. Chen, Y. Zhao, C. Liu, Q. Shang, Z. Zhu, D. Liu, TRPV1-mediated UCP2 upregulation ameliorates hyperglycemia-induced endothelial dysfunction, Cardiovasc Diabetol 12 (2013) 69.

[118] D. Yang, Z. Luo, S. Ma, W.T. Wong, L. Ma, J. Zhong, H. He, Z. Zhao, T. Cao, Z. Yan, D. Liu, W.J. Arendshorst, Y. Huang, M. Tepel, Z. Zhu, Activation of TRPV1 by dietary capsaicin improves endothelium-dependent vasorelaxation and prevents hypertension, Cell Metab 12(2) (2010) 130-41.

[119] J.C. Torres-Narvaez, V. Mondragon Ldel, E. Varela Lopez, I. Perez-Torres, J.A. Diaz Juarez, J. Suarez, G.P. Hernandez, Role of the transient receptor potential vanilloid type 1 receptor and stretch-activated ion channels in nitric oxide release from endothelial cells of the aorta and heart in rats, Exp Clin Cardiol 17(3) (2012) 89-94.

[120] C.A. Sand, A.D. Grant, M. Nandi, Vascular Expression of Transient Receptor Potential Vanilloid 1 (TRPV1), J Histochem Cytochem 63(6) (2015) 449-53.

[121] R. Inoue, L.J. Jensen, J. Shi, H. Morita, M. Nishida, A. Honda, Y. Ito, Transient receptor potential channels in cardiovascular function and disease, Circ Res 99(2) (2006) 119-31.

[122] J. Fruhwald, J. Camacho Londono, S. Dembla, S. Mannebach, A. Lis, A. Drews, U. Wissenbach, J. Oberwinkler, S.E. Philipp, Alternative splicing of a protein domain indispensable for function of transient receptor potential melastatin 3 (TRPM3) ion channels, J Biol Chem 287(44) (2012) 36663-72.

[123] C. Grimm, R. Kraft, S. Sauerbruch, G. Schultz, C. Harteneck, Molecular and functional characterization of the melastatin-related cation channel TRPM3, J Biol Chem 278(24) (2003) 21493-501.

[124] J. Oberwinkler, A. Lis, K.M. Giehl, V. Flockerzi, S.E. Philipp, Alternative splicing switches the divalent cation selectivity of TRPM3 channels, J Biol Chem 280(23) (2005) 22540-8. 
[125] N. Lee, J. Chen, L. Sun, S. Wu, K.R. Gray, A. Rich, M. Huang, J.H. Lin, J.N. Feder, E.B. Janovitz, P.C. Levesque, M.A. Blanar, Expression and characterization of human transient receptor potential melastatin 3 (hTRPM3), J Biol Chem 278(23) (2003) 20890-7.

[126] K. Held, T. Voets, J. Vriens, TRPM3 in temperature sensing and beyond, Temperature (Austin) 2(2) (2015) 201-13.

[127] C. Grimm, R. Kraft, G. Schultz, C. Harteneck, Activation of the melastatin-related cation channel TRPM3 by D-erythro-sphingosine [corrected], Mol Pharmacol 67(3) (2005) 798-805.

[128] J. Naylor, J. Li, C.J. Milligan, F. Zeng, P. Sukumar, B. Hou, A. Sedo, N. Yuldasheva, Y. Majeed, D. Beri, S. Jiang, V.A. Seymour, L. McKeown, B. Kumar, C. Harteneck, D. O'Regan, S.B. Wheatcroft, M.T. Kearney, C. Jones, K.E. Porter, D.J. Beech, Pregnenolone sulphate- and cholesterol-regulated TRPM3 channels coupled to vascular smooth muscle secretion and contraction, Circ Res 106(9) (2010) 1507-15.

[129] I. Straub, U. Krugel, F. Mohr, J. Teichert, O. Rizun, M. Konrad, J. Oberwinkler, M. Schaefer, Flavanones that selectively inhibit TRPM3 attenuate thermal nociception in vivo, Mol Pharmacol 84(5) (2013) 736-50.

[130] T.F. Wagner, S. Loch, S. Lambert, I. Straub, S. Mannebach, I. Mathar, M. Dufer, A. Lis, V. Flockerzi, S.E. Philipp, J. Oberwinkler, Transient receptor potential M3 channels are ionotropic steroid receptors in pancreatic beta cells, Nat Cell Biol 10(12) (2008) 1421-30.

[131] C. Klose, I. Straub, M. Riehle, F. Ranta, D. Krautwurst, S. Ullrich, W. Meyerhof, C. Harteneck, Fenamates as TRP channel blockers: mefenamic acid selectively blocks TRPM3, Br J Pharmacol 162(8) (2011) 1757-69.

[132] H. Havlikova, M. Hill, R. Hampl, L. Starka, Sex- and age-related changes in epitestosterone in relation to pregnenolone sulfate and testosterone in normal subjects, J Clin Endocrinol Metab 87(5) (2002) 2225-31.

[133] C. Harteneck, Pregnenolone sulfate: from steroid metabolite to TRP channel ligand, Molecules 18(10) (2013) 12012-28.

[134] A. Drews, F. Mohr, O. Rizun, T.F. Wagner, S. Dembla, S. Rudolph, S. Lambert, M. Konrad, S.E. Philipp, M. Behrendt, S. Marchais-Oberwinkler, D.F. Covey, J. Oberwinkler, Structural requirements of steroidal agonists of transient receptor potential melastatin 3 (TRPM3) cation channels, Br J Pharmacol 171(4) (2014) 1019-32.

[135] K. Held, T. Kichko, K. De Clercq, H. Klaassen, R. Van Bree, J.C. Vanherck, A. Marchand, P.W. Reeh, P. Chaltin, T. Voets, J. Vriens, Activation of TRPM3 by a potent synthetic ligand reveals a role in peptide release, Proc Natl Acad Sci U S A 112(11) (2015) E1363-72.

[136] Y. Majeed, S. Tumova, B.L. Green, V.A. Seymour, D.M. Woods, A.K. Agarwal, J. Naylor, S. Jiang, H.M. Picton, K.E. Porter, D.J. O'Regan, K. Muraki, C.W. Fishwick, D.J. Beech, Pregnenolone sulphate-independent inhibition of TRPM3 channels by progesterone, Cell Calcium 51(1) (2012) 1-11.

[137] I. Straub, F. Mohr, J. Stab, M. Konrad, S.E. Philipp, J. Oberwinkler, M. Schaefer, Citrus fruit and fabacea secondary metabolites potently and selectively block TRPM3, Br J Pharmacol 168(8) (2013) 1835-50.

[138] H. Suzuki, E. Sasaki, A. Nakagawa, Y. Muraki, N. Hatano, K. Muraki, Diclofenac, a nonsteroidal anti-inflammatory drug, is an antagonist of human TRPM3 isoforms, Pharmacol Res Perspect 4(3) (2016) e00232.

[139] U. Krugel, I. Straub, H. Beckmann, M. Schaefer, Primidone inhibits TRPM3 and attenuates thermal nociception in vivo, Pain 158(5) (2017) 856-867. 
[140] H. Xu, N.T. Blair, D.E. Clapham, Camphor activates and strongly desensitizes the transient receptor potential vanilloid subtype 1 channel in a vanilloid-independent mechanism, J Neurosci 25(39) (2005) 8924-37.

[141] E. Fonfria, P.R. Murdock, F.S. Cusdin, C.D. Benham, R.E. Kelsell, S. McNulty, Tissue distribution profiles of the human TRPM cation channel family, J Recept Signal Transduct Res 26(3) (2006) 159-78.

[142] S. Hughes, C.A. Pothecary, A. Jagannath, R.G. Foster, M.W. Hankins, S.N. Peirson, Profound defects in pupillary responses to light in TRPM-channel null mice: a role for TRPM channels in non-image-forming photoreception, Eur J Neurosci 35(1) (2012) 34-43.

[143] E. Kastenhuber, M. Gesemann, M. Mickoleit, S.C. Neuhauss, Phylogenetic analysis and expression of zebrafish transient receptor potential melastatin family genes, Dev Dyn 242(11) (2013) 1236-49.

[144] M. Karali, I. Peluso, V. Marigo, S. Banfi, Identification and characterization of microRNAs expressed in the mouse eye, Invest Ophthalmol Vis Sci 48(2) (2007) 509-15.

[145] S.L. Li, X.H. Wang, H.P. Wang, Z.H. Yang, W.C. Gao, X.Y. Pu, [Expression of TRPM and TRPV channel family mRNA in rat spermatogenic cells], Nan Fang Yi Ke Da Xue Xue Bao 28(12) (2008) 2150-3.

[146] W. Yu, W.G. Hill, G. Apodaca, M.L. Zeidel, Expression and distribution of transient receptor potential (TRP) channels in bladder epithelium, Am J Physiol Renal Physiol 300(1) (2011) F49-59.

[147] J. Naylor, C.J. Milligan, F. Zeng, C. Jones, D.J. Beech, Production of a specific extracellular inhibitor of TRPM3 channels, Br J Pharmacol 155(4) (2008) 567-73.

[148] J. Oberwinkler, S.E. Philipp, Trpm3, Handb Exp Pharmacol 222 (2014) 427-59.

[149] A.M. Peier, A. Moqrich, A.C. Hergarden, A.J. Reeve, D.A. Andersson, G.M. Story, T.J. Earley, I. Dragoni, P. Mclntyre, S. Bevan, A. Patapoutian, A TRP channel that senses cold stimuli and menthol, Cell 108(5) (2002) 705-15.

[150] D.M. Bautista, J. Siemens, J.M. Glazer, P.R. Tsuruda, A.I. Basbaum, C.L. Stucky, S.E. Jordt, D. Julius, The menthol receptor TRPM8 is the principal detector of environmental cold, Nature 448(7150) (2007) 204-8.

[151] R.W. Colburn, M.L. Lubin, D.J. Stone, Jr., Y. Wang, D. Lawrence, M.R. D'Andrea, M.R. Brandt, Y. Liu, C.M. Flores, N. Qin, Attenuated cold sensitivity in TRPM8 null mice, Neuron 54(3) (2007) 379-86.

[152] A. Dhaka, A.N. Murray, J. Mathur, T.J. Earley, M.J. Petrus, A. Patapoutian, TRPM8 is required for cold sensation in mice, Neuron 54(3) (2007) 371-8.

[153] I. Sarria, J. Ling, M.X. Zhu, J.G. Gu, TRPM8 acute desensitization is mediated by calmodulin and requires PIP(2): distinction from tachyphylaxis, J Neurophysiol 106(6) (2011) 3056-66.

[154] E. Bas, M. Naziroglu, L. Pecze, ADP-Ribose and oxidative stress activate TRPM8 channel in prostate cancer and kidney cells, Sci Rep 9(1) (2019) 4100.

[155] J.M. Yang, F. Li, Q. Liu, M. Ruedi, E.T. Wei, M. Lentsman, H.S. Lee, W. Choi, S.J. Kim, K.C. Yoon, A novel TRPM8 agonist relieves dry eye discomfort, BMC Ophthalmol 17(1) (2017) 101. [156] N. Aizawa, H. Ohshiro, S. Watanabe, H. Kume, Y. Homma, Y. Igawa, RQ-00434739, a novel TRPM8 antagonist, inhibits prostaglandin E2-induced hyperactivity of the primary bladder afferent nerves in rats, Life Sci 218 (2019) 89-95.

[157] H. Kume, M. Tsukimoto, TRPM8 channel inhibitor AMTB suppresses murine T-cell activation induced by T-cell receptor stimulation, concanavalin A, or external antigen restimulation, Biochem Biophys Res Commun 509(4) (2019) 918-924. 
[158] N. Aizawa, Y. Fujimori, J.I. Kobayashi, O. Nakanishi, H. Hirasawa, H. Kume, Y. Homma, Y. Igawa, KPR-2579, a novel TRPM8 antagonist, inhibits acetic acid-induced bladder afferent hyperactivity in rats, Neurourol Urodyn 37(5) (2018) 1633-1640.

[159] L. Misery, A. Santerre, A. Batardiere, N. Hornez, A.S. Nedelec, F. Le Caer, P. Bourgeois, F. Huet, G. Neufang, Real-life study of anti-itching effects of a cream containing menthoxypropanediol, a TRPM8 agonist, in atopic dermatitis patients, J Eur Acad Dermatol Venereol 33(2) (2019) e67-e69.

[160] C.D. Johnson, D. Melanaphy, A. Purse, S.A. Stokesberry, P. Dickson, A.V. Zholos, Transient receptor potential melastatin 8 channel involvement in the regulation of vascular tone, Am J Physiol Heart Circ Physiol 296(6) (2009) H1868-77.

[161] T. Kotaka, S. Kimura, M. Kashiwayanagi, J. Iwamoto, Camphor induces cold and warm sensations with increases in skin and muscle blood flow in human, Biol Pharm Bull 37(12) (2014) 1913-8.

[162] J. Sun, T. Yang, P. Wang, S. Ma, Z. Zhu, Y. Pu, L. Li, Y. Zhao, S. Xiong, D. Liu, Z. Zhu, Activation of cold-sensing transient receptor potential melastatin subtype 8 antagonizes vasoconstriction and hypertension through attenuating RhoA/Rho kinase pathway, Hypertension 63(6) (2014) 1354-63.

[163] M.K. McGahon, J.A. Fernandez, D.P. Dash, J. McKee, D.A. Simpson, A.V. Zholos, J.G. McGeown, T.M. Curtis, TRPV2 Channels Contribute to Stretch-Activated Cation Currents and Myogenic Constriction in Retinal Arterioles, Invest Ophthalmol Vis Sci 57(13) (2016) 56375647.

[164] P.W. Pires, M.N. Sullivan, H.A. Pritchard, J.J. Robinson, S. Earley, Unitary TRPV3 channel $\mathrm{Ca} 2+$ influx events elicit endothelium-dependent dilation of cerebral parenchymal arterioles, Am J Physiol Heart Circ Physiol 309(12) (2015) H2031-41.

[165] T.V. Murphy, A. Kanagarajah, S. Toemoe, P.P. Bertrand, T.H. Grayson, F.C. Britton, L. Leader, S. Senadheera, S.L. Sandow, TRPV3 expression and vasodilator function in isolated uterine radial arteries from non-pregnant and pregnant rats, Vascul Pharmacol 83 (2016) 6677.

[166] S. Earley, A.L. Gonzales, Z.I. Garcia, A dietary agonist of transient receptor potential cation channel V3 elicits endothelium-dependent vasodilation, Mol Pharmacol 77(4) (2010) 612-20.

[167] S. Earley, T.J. Heppner, M.T. Nelson, J.E. Brayden, TRPV4 forms a novel Ca2+ signaling complex with ryanodine receptors and BKCa channels, Circ Res 97(12) (2005) 1270-9.

[168] D. Peixoto-Neves, Q. Wang, J.H. Leal-Cardoso, L.V. Rossoni, J.H. Jaggar, Eugenol dilates mesenteric arteries and reduces systemic BP by activating endothelial cell TRPV4 channels, $\mathrm{Br}$ J Pharmacol 172(14) (2015) 3484-94.

[169] J. Mercado, R. Baylie, M.F. Navedo, C. Yuan, J.D. Scott, M.T. Nelson, J.E. Brayden, L.F. Santana, Local control of TRPV4 channels by AKAP150-targeted PKC in arterial smooth muscle, J Gen Physiol 143(5) (2014) 559-75.

[170] T. Ducret, C. Guibert, R. Marthan, J.P. Savineau, Serotonin-induced activation of TRPV4like current in rat intrapulmonary arterial smooth muscle cells, Cell Calcium 43(4) (2008) 31523.

[171] Y. Ma, P. Zhang, J. Li, J. Lu, J. Ge, Z. Zhao, X. Ma, S. Wan, X. Yao, B. Shen, Epoxyeicosatrienoic acids act through TRPV4-TRPC1-KCa1.1 complex to induce smooth muscle membrane hyperpolarization and relaxation in human internal mammary arteries, Biochim Biophys Acta 1852(3) (2015) 552-9. 
[172] T. Lewis, Observations on RESEARCH IN MEDICINE: ITS POSITION AND ITS NEEDS, Br Med J 1(3610) (1930) 479-83.

[173] R. Senaris, P. Ordas, A. Reimundez, F. Viana, Mammalian cold TRP channels: impact on thermoregulation and energy homeostasis, Pflugers Arch 470(5) (2018) 761-777.

[174] E.A. Tansey, C.D. Johnson, Recent advances in thermoregulation, Adv Physiol Educ 39(3) (2015) 139-48.

[175] J.M. Johnson, D.L. Kellogg, Jr., Local thermal control of the human cutaneous circulation, J Appl Physiol (1985) 109(4) (2010) 1229-38.

[176] H.A. Daanen, Finger cold-induced vasodilation: a review, Eur J Appl Physiol 89(5) (2003) 411-26.

[177] S.D. Brain, R.G. Petty, J.D. Lewis, T.J. Williams, Cutaneous blood flow responses in the forearms of Raynaud's patients induced by local cooling and intradermal injections of CGRP and histamine, Br J Clin Pharmacol 30(6) (1990) 853-9.

[178] J.M. Johnson, T.C. Yen, K. Zhao, W.A. Kosiba, Sympathetic, sensory, and nonneuronal contributions to the cutaneous vasoconstrictor response to local cooling, Am J Physiol Heart Circ Physiol 288(4) (2005) H1573-9.

[179] M.J. Caterina, Transient receptor potential ion channels as participants in thermosensation and thermoregulation, Am J Physiol Regul Integr Comp Physiol 292(1) (2007) R64-76.

[180] O. Caspani, P.A. Heppenstall, TRPA1 and cold transduction: an unresolved issue?, J Gen Physiol 133(3) (2009) 245-9.

[181] Y. Karashima, K. Talavera, W. Everaerts, A. Janssens, K.Y. Kwan, R. Vennekens, B. Nilius, T. Voets, TRPA1 acts as a cold sensor in vitro and in vivo, Proc Natl Acad Sci U S A 106(4) (2009) 1273-8.

[182] K.Y. Kwan, A.J. Allchorne, M.A. Vollrath, A.P. Christensen, D.S. Zhang, C.J. Woolf, D.P. Corey, TRPA1 contributes to cold, mechanical, and chemical nociception but is not essential for hair-cell transduction, Neuron 50(2) (2006) 277-89.

[183] C. Gentry, N. Stoakley, D.A. Andersson, S. Bevan, The roles of iPLA2, TRPM8 and TRPA1 in chemically induced cold hypersensitivity, Mol Pain 6 (2010) 4.

[184] A.A. Aubdool, X. Kodji, N. Abdul-Kader, R. Heads, E.S. Fernandes, S. Bevan, S.D. Brain, TRPA1 activation leads to neurogenic vasodilatation: involvement of reactive oxygen nitrogen species in addition to CGRP and NO, Br J Pharmacol 173(15) (2016) 2419-33.

[185] W.M. Knowlton, A. Bifolck-Fisher, D.M. Bautista, D.D. McKemy, TRPM8, but not TRPA1, is required for neural and behavioral responses to acute noxious cold temperatures and coldmimetics in vivo, Pain 150(2) (2010) 340-50.

[186] D. del Camino, S. Murphy, M. Heiry, L.B. Barrett, T.J. Earley, C.A. Cook, M.J. Petrus, M. Zhao, M. D'Amours, N. Deering, G.J. Brenner, M. Costigan, N.J. Hayward, J.A. Chong, C.M. Fanger, C.J. Woolf, A. Patapoutian, M.M. Moran, TRPA1 contributes to cold hypersensitivity, J Neurosci 30(45) (2010) 15165-74.

[187] L. Moparthi, T.I. Kichko, M. Eberhardt, E.D. Hogestatt, P. Kjellbom, U. Johanson, P.W. Reeh, A. Leffler, M.R. Filipovic, P.M. Zygmunt, Human TRPA1 is a heat sensor displaying intrinsic U-shaped thermosensitivity, Sci Rep 6 (2016) 28763.

[188] J. Chen, S.K. Joshi, S. DiDomenico, R.J. Perner, J.P. Mikusa, D.M. Gauvin, J.A. Segreti, P. Han, X.F. Zhang, W. Niforatos, B.R. Bianchi, S.J. Baker, C. Zhong, G.H. Simler, H.A. McDonald, R.G. Schmidt, S.P. McGaraughty, K.L. Chu, C.R. Faltynek, M.E. Kort, R.M. Reilly, P.R. Kym, Selective blockade of TRPA1 channel attenuates pathological pain without altering noxious cold sensation or body temperature regulation, Pain 152(5) (2011) 1165-72. 
[189] C. de Oliveira, A. Garami, S.G. Lehto, E. Pakai, V. Tekus, K. Pohoczky, B.D. Youngblood, W. Wang, M.E. Kort, P.R. Kym, E. Pinter, N.R. Gavva, A.A. Romanovsky, Transient receptor potential channel ankyrin-1 is not a cold sensor for autonomic thermoregulation in rodents, J Neurosci 34(13) (2014) 4445-52.

[190] A.A. Aubdool, R. Graepel, X. Kodji, K.M. Alawi, J.V. Bodkin, S. Srivastava, C. Gentry, R. Heads, A.D. Grant, E.S. Fernandes, S. Bevan, S.D. Brain, TRPA1 is essential for the vascular response to environmental cold exposure, Nat Commun 5 (2014) 5732.

[191] M.C. Almeida, T. Hew-Butler, R.N. Soriano, S. Rao, W. Wang, J. Wang, N. Tamayo, D.L. Oliveira, T.B. Nucci, P. Aryal, A. Garami, D. Bautista, N.R. Gavva, A.A. Romanovsky, Pharmacological blockade of the cold receptor TRPM8 attenuates autonomic and behavioral cold defenses and decreases deep body temperature, J Neurosci 32(6) (2012) 2086-99.

[192] W.M. Knowlton, R. Palkar, E.K. Lippoldt, D.D. McCoy, F. Baluch, J. Chen, D.D. McKemy, A sensory-labeled line for cold: TRPM8-expressing sensory neurons define the cellular basis for cold, cold pain, and cooling-mediated analgesia, J Neurosci 33(7) (2013) 2837-48.

[193] D.D. McKemy, TRPM8: The Cold and Menthol Receptor, in: W.B. Liedtke, S. Heller (Eds.), TRP Ion Channel Function in Sensory Transduction and Cellular Signaling Cascades, Boca Raton (FL), 2007.

[194] M.C. Almeida, A.A. Steiner, L.G. Branco, A.A. Romanovsky, Cold-seeking behavior as a thermoregulatory strategy in systemic inflammation, Eur J Neurosci 23(12) (2006) 3359-67.

[195] K. Tajino, K. Matsumura, K. Kosada, T. Shibakusa, K. Inoue, T. Fushiki, H. Hosokawa, S. Kobayashi, Application of menthol to the skin of whole trunk in mice induces autonomic and behavioral heat-gain responses, Am J Physiol Regul Integr Comp Physiol 293(5) (2007) R212835.

[196] Z. Winter, P. Gruschwitz, S. Eger, F. Touska, K. Zimmermann, Cold Temperature Encoding by Cutaneous TRPA1 and TRPM8-Carrying Fibers in the Mouse, Front Mol Neurosci 10 (2017) 209.

[197] J.O. Sosa-Pagan, E.S. Iversen, J. Grandl, TRPV1 temperature activation is specifically sensitive to strong decreases in amino acid hydrophobicity, Sci Rep 7(1) (2017) 549.

[198] A. Jancso-Gabor, J. Szolcsanyi, N. Jancso, Irreversible impairment of thermoregulation induced by capsaicin and similar pungent substances in rats and guinea-pigs, J Physiol 206(3) (1970) 495-507.

[199] Z. Szelenyi, Z. Hummel, J. Szolcsanyi, J.B. Davis, Daily body temperature rhythm and heat tolerance in TRPV1 knockout and capsaicin pretreated mice, Eur J Neurosci 19(5) (2004) 14214.

[200] S. McGaraughty, J.A. Segreti, R.M. Fryer, B.S. Brown, C.R. Faltynek, P.R. Kym, Antagonism of TRPV1 receptors indirectly modulates activity of thermoregulatory neurons in the medial preoptic area of rats, Brain Res 1268 (2009) 58-67.

[201] R.L. Baylie, J.E. Brayden, TRPV channels and vascular function, Acta Physiol (Oxf) 203(1) (2011) 99-116.

[202] M.J. Caterina, T.A. Rosen, M. Tominaga, A.J. Brake, D. Julius, A capsaicin-receptor homologue with a high threshold for noxious heat, Nature 398(6726) (1999) 436-41.

[203] U. Park, N. Vastani, Y. Guan, S.N. Raja, M. Koltzenburg, M.J. Caterina, TRP vanilloid 2 knock-out mice are susceptible to perinatal lethality but display normal thermal and mechanical nociception, J Neurosci 31(32) (2011) 11425-36.

[204] R.A. Nicholas, W.C. Watt, E.R. Lazarowski, Q. Li, K. Harden, Uridine nucleotide selectivity of three phospholipase C-activating P2 receptors: identification of a UDP-selective, a UTPselective, and an ATP- and UTP-specific receptor, Mol Pharmacol 50(2) (1996) 224-9. 
[205] L.H. Wang, M. Luo, Y. Wang, J.J. Galligan, D.H. Wang, Impaired vasodilation in response to perivascular nerve stimulation in mesenteric arteries of TRPV1-null mutant mice, J Hypertens 24(12) (2006) 2399-408.

[206] C.A. Maggi, Tachykinins and calcitonin gene-related peptide (CGRP) as co-transmitters released from peripheral endings of sensory nerves, Prog Neurobiol 45(1) (1995) 1-98.

[207] D. van Rossum, U.K. Hanisch, R. Quirion, Neuroanatomical localization, pharmacological characterization and functions of CGRP, related peptides and their receptors, Neurosci Biobehav Rev 21(5) (1997) 649-78.

[208] A. Fujimori, A. Saito, S. Kimura, T. Watanabe, Y. Uchiyama, H. Kawasaki, K. Goto, Neurogenic vasodilation and release of calcitonin gene-related peptide (CGRP) from perivascular nerves in the rat mesenteric artery, Biochem Biophys Res Commun 165(3) (1989) 1391-8.

[209] R. Uddman, T. Grunditz, F. Sundler, Calcitonin gene related peptide: a sensory transmitter in dental pulps?, Scand J Dent Res 94(3) (1986) 219-24.

[210] H.G. Knaus, O.B. McManus, S.H. Lee, W.A. Schmalhofer, M. Garcia-Calvo, L.M. Helms, M. Sanchez, K. Giangiacomo, J.P. Reuben, A.B. Smith, 3rd, et al., Tremorgenic indole alkaloids potently inhibit smooth muscle high-conductance calcium-activated potassium channels, Biochemistry 33(19) (1994) 5819-28.

[211] J.P. Felix, R.M. Bugianesi, W.A. Schmalhofer, R. Borris, M.A. Goetz, O.D. Hensens, J.M. Bao, F. Kayser, W.H. Parsons, K. Rupprecht, M.L. Garcia, G.J. Kaczorowski, R.S. Slaughter, Identification and biochemical characterization of a novel nortriterpene inhibitor of the human lymphocyte voltage-gated potassium channel, Kv1.3, Biochemistry 38(16) (1999) 4922-30.

[212] P. Escoubas, S. Diochot, M.L. Celerier, T. Nakajima, M. Lazdunski, Novel tarantula toxins for subtypes of voltage-dependent potassium channels in the Kv2 and Kv4 subfamilies, Mol Pharmacol 62(1) (2002) 48-57.

[213] M.P. Jankowski, K.M. Baumbauer, T. Wang, K.M. Albers, B.M. Davis, H.R. Koerber, Cutaneous neurturin overexpression alters mechanical, thermal, and cold responsiveness in physiologically identified primary afferents, J Neurophysiol 117(3) (2017) 1258-1265.

[214] L.B. Rowell, Reflex control of regional circulations in humans, J Auton Nerv Syst 11(2) (1984) 101-14.

[215] H. Watanabe, J. Vriens, S.H. Suh, C.D. Benham, G. Droogmans, B. Nilius, Heat-evoked activation of TRPV4 channels in a HEK293 cell expression system and in native mouse aorta endothelial cells, J Biol Chem 277(49) (2002) 47044-51.

[216] Z. Yue, J. Xie, A.S. Yu, J. Stock, J. Du, L. Yue, Role of TRP channels in the cardiovascular system, Am J Physiol Heart Circ Physiol 308(3) (2015) H157-82.

[217] S. Earley, J.E. Brayden, Transient receptor potential channels in the vasculature, Physiol Rev 95(2) (2015) 645-90.

[218] T.I. Kichko, P.W. Reeh, TRPV1 controls acid- and heat-induced calcitonin gene-related peptide release and sensitization by bradykinin in the isolated mouse trachea, Eur J Neurosci 29(9) (2009) 1896-904.

[219] D.W. Gray, I. Marshall, Human alpha-calcitonin gene-related peptide stimulates adenylate cyclase and guanylate cyclase and relaxes rat thoracic aorta by releasing nitric oxide, Br J Pharmacol 107(3) (1992) 691-6.

[220] X. Yu, F. Li, E. Klussmann, J.N. Stallone, G. Han, G protein-coupled estrogen receptor 1 mediates relaxation of coronary arteries via cAMP/PKA-dependent activation of MLCP, Am J Physiol Endocrinol Metab 307(4) (2014) E398-407. 
[221] J.G. De Mey, R. Megens, G.E. Fazzi, Functional antagonism between endogenous neuropeptide $Y$ and calcitonin gene-related peptide in mesenteric resistance arteries, J Pharmacol Exp Ther 324(3) (2008) 930-7.

[222] R. Aoki, U. Yokoyama, Y. Ichikawa, M. Taguri, S. Kumagaya, R. Ishiwata, C. Yanai, S. Fujita, M. Umemura, T. Fujita, S. Okumura, M. Sato, S. Minamisawa, T. Asou, M. Masuda, S. Iwasaki, S. Nishimaki, K. Seki, S. Yokota, Y. Ishikawa, Decreased serum osmolality promotes ductus arteriosus constriction, Cardiovasc Res 104(2) (2014) 326-36.

[223] G. Santoni, C. Cardinali, M.B. Morelli, M. Santoni, M. Nabissi, C. Amantini, Danger- and pathogen-associated molecular patterns recognition by pattern-recognition receptors and ion channels of the transient receptor potential family triggers the inflammasome activation in immune cells and sensory neurons, J Neuroinflammation 12 (2015) 21.

[224] V. Meseguer, Y.A. Alpizar, E. Luis, S. Tajada, B. Denlinger, O. Fajardo, J.A. Manenschijn, C. Fernandez-Pena, A. Talavera, T. Kichko, B. Navia, A. Sanchez, R. Senaris, P. Reeh, M.T. PerezGarcia, J.R. Lopez-Lopez, T. Voets, C. Belmonte, K. Talavera, F. Viana, TRPA1 channels mediate acute neurogenic inflammation and pain produced by bacterial endotoxins, Nat Commun 5 (2014) 3125.

[225] B. Boonen, Y.A. Alpizar, A. Sanchez, A. Lopez-Requena, T. Voets, K. Talavera, Differential effects of lipopolysaccharide on mouse sensory TRP channels, Cell Calcium 73 (2018) 72-81.

[226] F. Viana, TRPA1 channels: molecular sentinels of cellular stress and tissue damage, J Physiol 594(15) (2016) 4151-69.

[227] A. Czikora, I. Rutkai, E.T. Pasztor, A. Szalai, R. Porszasz, J. Boczan, I. Edes, Z. Papp, A. Toth, Different desensitization patterns for sensory and vascular TRPV1 populations in the rat: expression, localization and functional consequences, PLoS One 8(11) (2013) e78184.

[228] X. Qian, M. Francis, V. Solodushko, S. Earley, M.S. Taylor, Recruitment of dynamic endothelial $\mathrm{Ca} 2+$ signals by the TRPA1 channel activator AITC in rat cerebral arteries, Microcirculation 20(2) (2013) 138-48.

[229] C. Munns, M. AlQatari, M. Koltzenburg, Many cold sensitive peripheral neurons of the mouse do not express TRPM8 or TRPA1, Cell Calcium 41(4) (2007) 331-42.

[230] D.D. McKemy, The molecular and cellular basis of cold sensation, ACS Chem Neurosci $4(2)(2013)$ 238-47.

[231] A. Reimundez, C. Fernandez-Pena, G. Garcia, R. Fernandez, P. Ordas, R. Gallego, J.L. Pardo-Vazquez, V. Arce, F. Viana, R. Senaris, Deletion of the Cold Thermoreceptor TRPM8 Increases Heat Loss and Food Intake Leading to Reduced Body Temperature and Obesity in Mice, J Neurosci 38(15) (2018) 3643-3656.

[232] A. Babes, D. Zorzon, G. Reid, Two populations of cold-sensitive neurons in rat dorsal root ganglia and their modulation by nerve growth factor, Eur J Neurosci 20(9) (2004) 2276-82.

[233] K. Kobayashi, T. Fukuoka, K. Obata, H. Yamanaka, Y. Dai, A. Tokunaga, K. Noguchi, Distinct expression of TRPM8, TRPA1, and TRPV1 mRNAs in rat primary afferent neurons with adelta/c-fibers and colocalization with trk receptors, J Comp Neurol 493(4) (2005) 596-606.

[234] G. Terenghi, S.Q. Zhang, W.G. Unger, J.M. Polak, Morphological changes of sensory CGRP-immunoreactive and sympathetic nerves in peripheral tissues following chronic denervation, Histochemistry 86(1) (1986) 89-95.

[235] W.J. Janssens, T.J. Verbeuren, P.M. Vanhoutte, Effect of moderate cooling on adrenergic neuroeffector interaction in canine cutaneous veins, Blood Vessels 18(6) (1981) 281-95.

[236] C.A. Gardner, R.C. Webb, Cold-induced vasodilatation in isolated, perfused rat tail artery, Am J Physiol 251(1 Pt 2) (1986) H176-81. 
[237] M.P. Smith, D. Beacham, E. Ensor, M. Koltzenburg, Cold-sensitive, menthol-insensitive neurons in the murine sympathetic nervous system, Neuroreport 15(9) (2004) 1399-403.

[238] S.T. Valdovinos, G.J. Landry, Raynaud syndrome, Tech Vasc Interv Radiol 17(4) (2014) 241-6.

[239] K.K. Temprano, A Review of Raynaud's Disease, Mo Med 113(2) (2016) 123-6.

[240] J. Holmberg, A. Bhattachariya, A. Alajbegovic, C. Rippe, M. Ekman, D. Dahan, T.T. Hien, T. Boettger, T. Braun, K. Sward, P. Hellstrand, S. Albinsson, Loss of Vascular Myogenic Tone in miR-143/145 Knockout Mice Is Associated With Hypertension-Induced Vascular Lesions in Small Mesenteric Arteries, Arterioscler Thromb Vasc Biol 38(2) (2018) 414-424.

[241] P.W. Pires, W.F. Jackson, A.M. Dorrance, Regulation of myogenic tone and structure of parenchymal arterioles by hypertension and the mineralocorticoid receptor, Am J Physiol Heart Circ Physiol 309(1) (2015) H127-36.

[242] N.F. Renna, N. de Las Heras, R.M. Miatello, Pathophysiology of vascular remodeling in hypertension, Int J Hypertens 2013 (2013) 808353.

[243] M.J. Tipton, F.S. Golden, C. Higenbottam, I.B. Mekjavic, C.M. Eglin, Temperature dependence of habituation of the initial responses to cold-water immersion, Eur J Appl Physiol Occup Physiol 78(3) (1998) 253-7.

[244] M.J. Tipton, The initial responses to cold-water immersion in man, Clin Sci (Lond) 77(6) (1989) 581-8.

[245] A.S. Borowiec, B. Sion, F. Chalmel, D.R. A, L. Lemonnier, T. De Clerck, A. Bokhobza, S. Derouiche, E. Dewailly, C. Slomianny, C. Mauduit, M. Benahmed, M. Roudbaraki, B. Jegou, N. Prevarskaya, G. Bidaux, Cold/menthol TRPM8 receptors initiate the cold-shock response and protect germ cells from cold-shock-induced oxidation, FASEB J 30(9) (2016) 3155-70. 
ABSTRACt 


\subsection{Abstract}

The regulation of the blood pressure is an essential physiological process mainly regulated by the vascular tone that is dependent on the function of multiple ion channels that determine the dynamics of intracellular $\mathrm{Ca}^{2+}$ concentration in VSMC, endothelial cells, as well as in perivascular nerves. Recent studies indicate that sensory TRP channels expressed in perivascular nerves may be implicated in the mechanisms underlying arterial tone regulation through the release of many factors such as CGRP and NA due to their capacity to influence cellular excitability and intracellular $\mathrm{Ca}^{2+}$ signaling. The vascular function of these channels has been associated to their chemo- and thermosensory properties, but their actual relevance to vascular tone regulation under external stimuli remain unknown.

Within the TRPM family, the role of TRPM3 channels in the contribution to vascular tone in resistance arteries has not been yet stablished. Therefore, using anatomical localization by immunofluorescence microscopy in intact resistance arteries and patch-clamp recordings in isolated VSMC, we found that TRPM3 expression in mesenteric arteries is restricted to perivascular sensory nerves. Pressure myography experiments showed that chemical activation of TRPM3 channels in mesenteric arteries by an endogenous steroid PS leads to vasodilatation, via CGRP release from perivascular sensory nerves. This data support the contribution of TRPM3 as a potential therapeutic target for the modulation of the resistance arteries tone and as a plausible effector of endogenous damage-associated molecules mediating protective responses in these vascular beds.

On the other hand, sensory TRP channels have also been involved in modulating the vascular function due to their involvement in sensing environmental stimuli, being actual sensor of thermal challenges. Thus, we examine the role of sensory TRPA1 and TRPM 8 channels and the underlying mechanism in a local vascular response induced by low temperatures. Using pressure myography experiments to determine changes in arterial diameter, we showed that TRPA1 and TRPM8 channels are involved in an intrinsic cold-induced vascular response in peripheral arteries and the global cold-induced potent vasoconstriction observed is the sum of the activation of these channels in sensory and sympathetic nerve endings. These results represent the first evidence for an intrinsic response to cold in cutaneous arteries and for the functional expression of TRPA1 and TRPM8 channels in efferent nerve fibers, suggesting them as the most potent targets in the treatment of cold-dependent peripheral vascular diseases. 
Altogether, we concluded that several sensory TRP channels play essential roles in the regulation of vascular tone in response to chemical and thermal stimuli.

\subsection{Resumen}

La regulación de la presión arterial es un proceso fisiológico esencial principalmente regulado por el tono vascular el cual depende de la función de múltiples canales iónicos que determinan la dinámica en la concentración del calcio intracelular de las células de músculo liso, las células endoteliales y los nervios perivasculares. Estudios recientes indican que los canales TRP sensoriales expresados en los nervios perivasculares pueden estar implicados en los mecanismos subyacentes a la regulación del tono arterial a través de la liberación de neuropeptidos tales como el CGRP y la noradrenalina debido a su capacidad para influir la excitabilidad celular y la señalización intracelular del calcio. La función vascular de estos canales está asociada a sus propiedades quimio- y termo- sensoriales, sin embargo, su importancia en la regulación del tono vascular frente a estímulos externos sigue siendo desconocida.

Dentro de la familia TRPM, el papel de los canales TRPM3 en la contribución al tono vascular en las arterias de resistencia aún no se ha establecido. Por lo tanto, al estudiar la localización anatómica de estos canales mediante el uso de la microscopía de inmunofluorescencia en arterias de resistencia y patch clamp en células de músculo liso aisladas, encontramos que la expresión de TRPM3 en arterias mesentéricas está restringida a los nervios sensoriales perivasculares. Los experimentos de miografía de presión mostraron que la activación química de los canales TRPM3 en las arterias mesentéricas mediante el empleo de un esteroide endógeno llamado PS conduce a la vasodilatación de la arteria mediante la liberación de CGRP de los nervios sensoriales perivasculares. Estos datos sobre la contribución de TRPM3 identifican a estos canales como dianas terapéuticas potenciales para la modulación del tono de las arterias de resistencia y como efectores de moléculas asociadas al daño endógeno que median las respuestas protectoras en los lechos vasculares.

Por otro lado, algunos canales sensoriales de la familia TRP también se han involucrado en la modulación de la función vascular debido a su participación en la detección de estímulos ambientales, identificándose como los sensores de los estímulos térmicos. Por lo tanto, examinamos los papeles de los canales sensoriales TRPA1 y TRPM8 y el mecanismo 
subyacente de la respuesta vascular intrínseca y local inducida por bajas temperaturas. Usando experimentos de miografía de presión para determinar los cambios en el diámetro arterial, demostramos que los canales TRPA1 y TRPM8 están involucrados en una respuesta vascular intrínseca inducida por el frío en arterias periféricas. Además, la vasoconstricción inducida por frío observada es la suma de la activación de estos canales que estarían presentes en las terminaciones nerviosas sensoriales y simpáticas. Estos resultados representan la primera evidencia de una respuesta intrínseca al frío en las arterias cutáneas además de una expresión funcional de los canales TRPA1 y TRPM8 en las fibras nerviosas simpáticas, lo que los sugiere como posibles objetivos potentes en el tratamiento de las enfermedades vasculares periféricas dependientes del frío. Finalmente, se llegó a la conclusión de que algunos canales sensoriales de la familia TRP desempeñan funciones de gran relevancia en la regulación del tono vascular frente a estímulos químicos y térmicos.

\section{Acknowledgements, Personal Contribution and Conflict of Interest Statements}

\section{Acknowledgements}

We thank Silvia Pinto, Evy Van Den Broek, Nele Van Ranst, Annelies Janssens, Melissa Benoit, Sara Kerselaers, Luke Grammet, Raf Verbist and Esperanza Alonso for excenllent technical assistance and Dr. Andrei Segal for technological support. The Cell Imaging Core facility of the KU Leuven for the use of the confocal microscope. All members of the LICR and Laboratory C2 for helpful discussions and Prof. Christian Alkaejer for the nice welcome to his laboratory in Aarhus University (Denmark).

\section{Personal contributions}

The contribution of Lucía Alonso-Carbajo to the manuscript in this thesis is:

Chapter 4: All experiments in Figures 4.1 and 4.7 to 4.16 . Conceiving, designing and analyzing of all experiments.

Chapter 5: All experiments in Figures 5.1 to 5.7. Conceiving, designing and analyzing of all experiments.

\section{Conflict of interest}

The authors of this thesis declare no competing interests. 


\section{LIST OF PUBLICATIONS AND Curriculum VitaE}




\subsection{List of Publications}

\section{Articles in international peer-reviewed journals}

- Alonso-Carbajo L., Alpizar Y. A., Startek J. B., Lopez-Lopez J. R., Perez-García M. T. and Talavera K. Activation of the cation cannel TRPM3 in perivascular nerves induces vasodilation of resistance arteries. Journal of Molecular and Cellular Cardiology (2019).

- Alonso-Carbajo L., Kecskes M., Jacobs G., Pironet A., Syam N., Talavera K., Vennekens R. Muscling in on TRP channels in vascular smooth muscle cells and cardiomyocytes, Cell Calcium 66 (2017) 48-61.

- Alonso-Carbajo L.*, Alvarez-Collado J.*, López-Medina A. I., Alpizar Y. A., Tajada S., Nilius B., Voets T., López-López J. R., Talavera K., Pérez-García M. T., Alvarez J.L. Cinnamaldehyde inhibits L-type calcium channels in mouse ventricular cardiomyocytes and vascular smooth muscle cells. Pflugers Arch. (2014); 466(11):2089-99 (*shared first author).

\section{Oral and poster communications in scientific meetings}

- Alonso-Carbajo L., Alpizar Y. A., Startek J. B., Lopez-Lopez J. R., Perez-Garcia M. T. and Talavera K. Activation of the cation channel TRPM3 in perivascular nerves induces vasodilation of resistance arteries. Reunión española de Canales lónicos. RECI VII. Cáceres, Spain, 15-17 May 2019 (Oral communication).

- Alonso-Carbajo L., Lopez-Lopez J. R., Perez-Garcia M. T. and Talavera K. Role of TRPA1 and TRPM8 channels in intrinsic vascular responses to cold. Europhysiology 2018. London, UK, 14-16 September 2018 (Oral communication).

- Alonso-Carbajo L., López-López J. R., Pérez-García M. T. and Talavera K. The role of TRPA1 and TRPM8 channels in vascular responses to cold. Reunión española de Canales lónicos. RECI VI., Santiago de Compostela, Spain, 6-8 September 2017 (Oral communication).

- Alonso-Carbajo L., Alpizar Y. A., Startek J. B., Lopez-Lopez J. R., Perez-Garcia M. T. and Talavera K. TRPM3 dependent-vasodilation in resistance arteries. $12^{\text {th }}$ International Symposium on Resistance Arteries (ISRA). Manchester, UK, 3-6 September 2017. Journal of Vascular Research 54(Suppl2) p57; (Oral communication).

- Alonso-Carbajo L., Lopez-Lopez J. R., Perez-Garcia M. T. and Talavera K. Pharmacological and genetic dissection of the role of TRPA1 and TRPM8 channels in intrinsic vascular responses to cold. 37th Congreso de la Sociedad Española de Farmacologia and British Pharmacological Society, Barcelona, Spain, 18-21 June, 2017, Basic and Clinical Pharmacology and Toxicology 121(Supplement 2) post72, p58; (Poster communication).

- Alonso-Carbajo L., Alpizar Y. A., Lopez-Lopez J. R., Perez-Garcia M. T. and Talavera K. Activation of TRPM3 in perivascular sensory nerves induces dilation of mouse 
resistance arteries. Spring meeting - Belgian Society of Physiology and Pharmacology. Brussels, Belgium, 22 April, 2017 (Poster communication).

- Alonso-Carbajo L., Alpizar Y. A., Lopez-Lopez J. R., Perez-Garcia M. T. and Talavera K. Activation of TRPM3 in perivascular sensory nerves induces dilation of mouse resistance arteries. $61^{\text {th }}$ Meeting of the Biophysical Society. New Orleans, USA, 11-15 February 2017, Biophysical Journal 112(3) Suppl. 1992-Pos, p404a, (Poster communication).

- Alonso-Carbajo L., Startek J. B., Lopez-Lopez J. R., Perez-Garcia M. T. and Talavera K. Trpm3 in perivascular sensory nerves contributes to the vascular tone in mouse resistance arteries. Regulation of Cell Functions by Transient Receptor Potential Channels. Herrsching, Munich, Germany, 28 September-1 October 2016 (Poster communication).

- Alonso-Carbajo L., Startek J. B., Lopez-Lopez J. R., Perez-Garcia M. T. and Talavera K. Activation of Trpm3 in perivascular sensory nerves induces dilation of mouse mesenteric arteries. Smooth Muscle, FASEB Science Research Conferences. Lisbon, Portugal, 17-22 July 2016 (Poster communication).

- Alonso-Carbajo L., Alpizar Y. A., Lopez-Lopez J. R., Perez-Garcia M. T. and Talavera K. Expression and functional contribution of TRPM 3 channels to vascular tone in essential hypertension. Pharmacology 2015. London, UK, 15-17 December 2015 (Poster communication).

- Alonso-Carbajo L., Alpizar Y. A., Voets T., López-López J. R., Pérez-García M. T. and Talavera, K. Expression, modulation and functional contribution of TRPM3 channels to vascular tone in essential hypertension. Reunión española de Canales lónicos. RECI V Barcelona, Spain, 4-6 October 2015 (Poster communication).

- Alonso-Carbajo L., Alpizar Y. A., Voets T., López-López J. R., Pérez-García M. T. and Talavera, K. Expression and functional contribution of TRPM3 channels to vascular tone in essential hypertension. $3^{\text {rd }}$ Leuven TRP symposium. Leuven, Belgium, 16-18 September 2015 (Poster communication).

- Alonso-Carbajo L., Alvarez-Collado J., López-Medina A. I., Alpizar Y. A., Tajada S., Nilius B., Voets T., López-López J. R., Talavera K., Pérez-García M. T. and Alvarez J. L. Cinnamaldehyde inhibits L-type calcium channels in mouse ventricular cardiomyocytes and vascular smooth muscle cells. Permeation and Gating of Ion Channels, Strobl am Wolfgangsee, Austria, 20-22 June 2014 (Poster communication). 


\subsection{Curriculum Vitae}

PERSONAL INFORMATION Lucía Alonso Carbajo

lucia.alonso@outlook.com

WORK EXPERIENCE

2013- 2019

EDUCATION AND TRAINING

2014-2019 Double degree PhD in Biomedical Sciences KU Leuven and University of Valladolid

Department of Cellular and Molecular Medicine/ VIB Center for Brain and Disease

Research (KU Leuven) and Department of Physiology (University of Valladolid)

$2012-2013$ Master of biomedical research

Valladolid University / IBGM / CSIC, Valladolid, Spain

$2006-2011$

$2011-2012$

Computer skills

Laboratory skills

- Primary cell cultures (vascular smooth muscle cells)

- Electrophysiology (patch clamp and automated patch-clamp)

- Telemetry technique (implantation of catheters in mice)

- Animal work (intraperitoneal and subcutaneous injections, welfare measurements)

- Tail-cuff method

- Calcium Imaging

- Pressure and wire myography

- Molecular biology (DNA and RNA purification, qPCR, genotyping)

- Alonso-Carbajo, L, Alpizar YA, Startek JB, Lopez-Lopez JR, Perez-Garcia MT and Talavera $\mathrm{K}$. Activation of the cation channel TRPM3 in perivascular nerves induces vasodilation of resistance arteries. J Mol Cell Cardiol. 2019 Mar; S0022-2828(19)30052-5

- Alonso-Carbajo, L, Alpizar YA, Knesces M, Jacobs G, Pironet, A, Syam N, Talavera K, and Vennekens R. Muscling in on TRP channels in vascular smooth muscle cells and cardiomyocytes. Cell calcium 2017 sep; 66:48-61

- Alonso-Carbajo L*, Alvarez-Collazo J*, Lopez-Medina Al, Alpizar YA, Tajada S, Nilius B, Voets T, Lopez-Lopez JR, Talavera K, Perez-Garcia MT, Alvarez JL. Cinnamaldehyde inhibits L-type calcium channels in mouse ventricular cardiomyocytes. Pflugers Arch-Eur J Physiol 2014 Nov; 466:2089-2099 (*first-shared authorship)

Projects

- Synthesis characterization and catalytic tests of metal-organic frameworks with secondary 
metal sites"

Research group: COMOC- ChemTech.

Coordinator: Prof. Dr. P. Van der Voort and Dr. Ying-Ya Liu.

Country: Ghent, Belgium

- "Synthesis and application of new hybrid imidate - phophane ligands" Research group: (Bio)- Organic Synthesis - Chem Tech

Coordinator: Prof. Dr. Van der Eycken and Dr. Katrien Bert

Country: Ghent, Belgium

- "Application of LA-ICP-MS for multi-elemental analysis of kidney stones" Research group: Atoom-en massaspectrometrie

Coordinator: Prof. Dr. P. Vanhaecke and Dr Andrei Izmer.

Country: Ghent, Belgium

- "Effects of cinnamaldehyde in L-type calcium channels of the vascular smooth muscle cells."

Research group: Electrophysiology group, Institute of Genetic and Molecular Biology

Coordinator: Prof. Dr. Jose Ramón López López and Prof. Dr. Mª Teresa Perez García

Country: Valladolid, Spain

Workshops, internships and scholarships

- Workshop in Hatfield (30 June- 1July, Hatfield, London, UK)

- Internship in Aarhus University, Department of Biomedicine (Noviembre 2017)

- Scholarship: First iniciatives

- Erasmus + practicas, Universidad de Valladolid

Courses - Statistics course in KU Leuven, Belgium (September - December 2014)

- Scientific Integrity course in KU Leuven, Belgium (15 May 2014)

- Course of HSE in Laboratories in KU Leuven, Belgium (7 March 2014)

- Animal Lab Certificate from KU Leuven, Belgium (February 2014) 
